\title{
Mikrocharakterisierung nanokristalliner Nickel-Phosphor- und Eisen-Silber-Legierungen mit der Tomographischen Atomsonde
}

Dissertation

zur Erlangung des Doktorgrades

der Mathematisch-Naturwissenschaftlichen Fakultäten

der Georg-August-Universität zu Göttingen

vorgelegt von

Boye Färber

aus Heide 
D 7

Referent:

Prof. Dr. R. Kirchheim

Korreferent:

Prof. Dr. K. Samwer

Tag der mündlichen Prüfung: $\quad$ 27. April 2000 


\section{Inhaltsverzeichnis}

1 Einleitung 1

2 Experimente 5

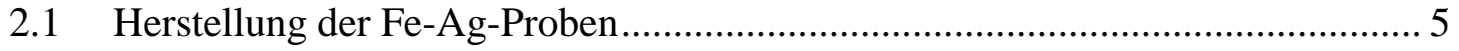

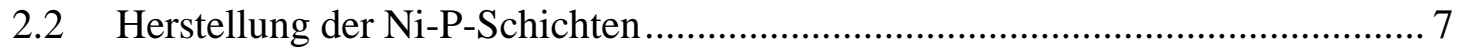

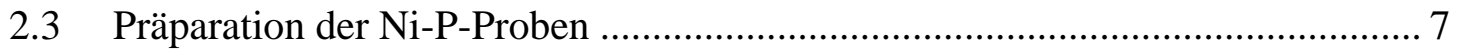

2.4 Konventionelle und Tomographische Atomsonde ......................................... 9

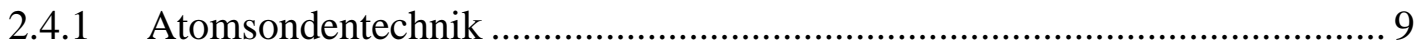

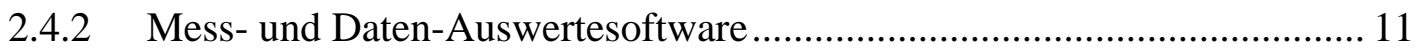

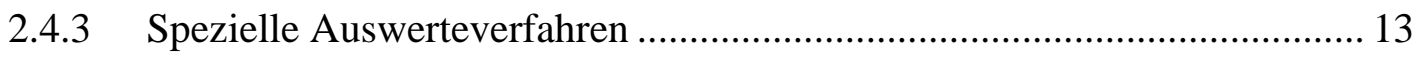

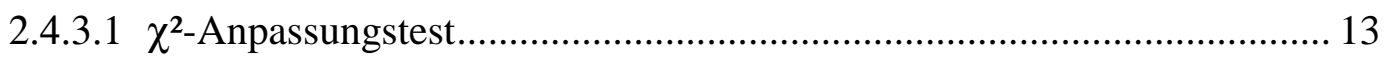

2.4.3.2 Konzentrationsprofile ................................................................... 14

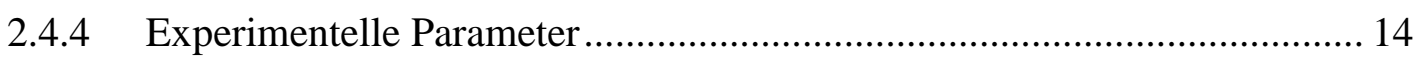

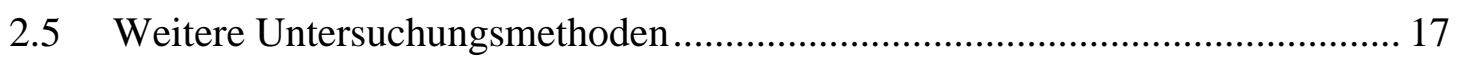

3 Untersuchungen an Fe-Ag-Legierungen 19

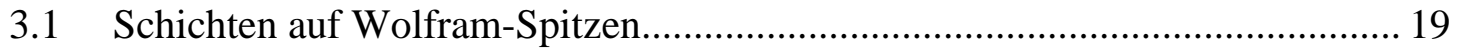

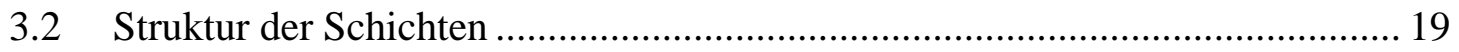

3.3 Chemische Zusammensetzung der Schichten.................................................. 22

3.4 Sputtereffekte bei der gepulsten Laserdeposition ........................................... 25

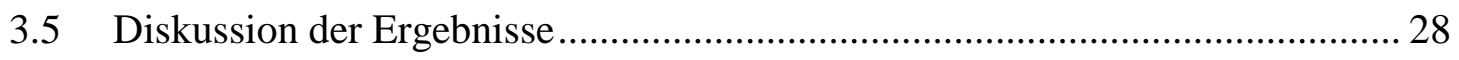

4 Untersuchungen an Ni-P-Legierungen 35

4.1 Mikrostruktur der Ni-P-Legierungen im Abscheidezustand ............................. 35

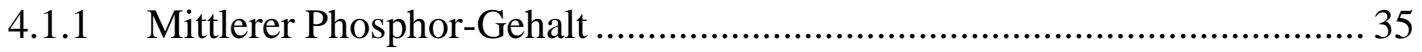

4.1.2 Korngrößen und Phasenzusammensetzung.............................................. 35

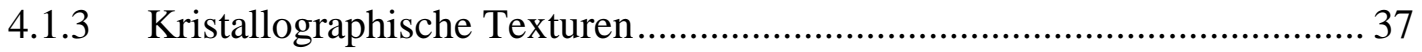

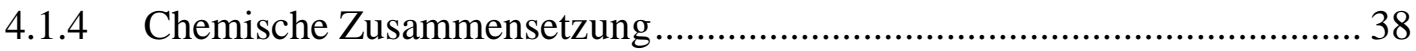

4.2 Entwicklung der Mikrostruktur durch Wärmebehandlung ............................... 40

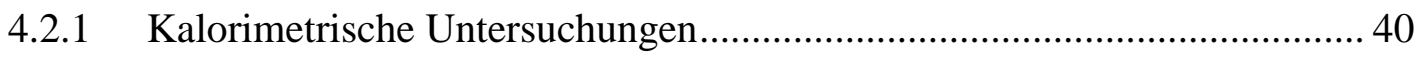

4.2.2 Entwicklung der Korngrößen und Phasenbildung ................................... 41

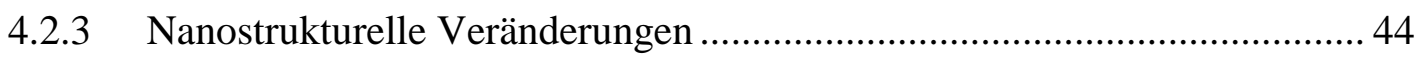

4.2.3.1 Untersuchungen an Nickel-3,6 At.\% Phosphor ..................................... 45

4.2.3.2 Untersuchungen an Nickel-5,9 At.\% Phosphor .................................... 49

4.2.3.3 Untersuchungen an Nickel-11,8 At.\% Phosphor .................................. 51

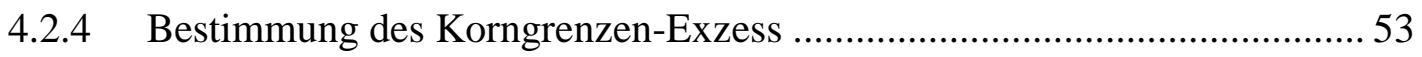




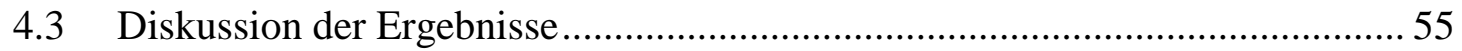

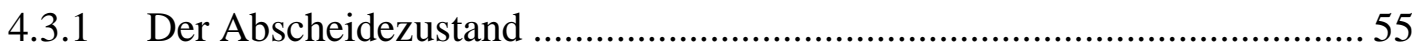

4.3.2 Der Einfluss von Wärmebehandlungen ................................................... 56

4.3.3 Thermische Stabilisierung durch Phosphor-Segregation ............................ 59

5 Zusammenfassung $\quad 65$

$\begin{array}{ll}\text { Symbolverzeichnis } & 67\end{array}$

$\begin{array}{ll}\text { Literaturverzeichnis } & 69\end{array}$

$\begin{array}{ll}\text { Anhang A } & \mathbf{7 3}\end{array}$

TRIM-Simulationen zu Fe-Ag-Schichten

Ergebnisse der TRIM-Simulationen

$\begin{array}{ll}\text { Anhang B } & \mathbf{7 7}\end{array}$

Deposition von Fe-Ag-Schichten unter Argon-Atmosphäre

Anhang C

Bestimmung von Phosphor-Konzentrationen in Korngrenzen 


\section{Einleitung}

Die Eigenschaften von metallischen Werkstoffen, insbesondere mechanische, chemische, elektrische und magnetische Eigenschaften, werden im wesentlichen durch ihre Mikrostruktur bestimmt. Bei der Werkstoffherstellung wird durch gezielte Anpassung der Mikrostruktur, häufig durch Wärmebehandlungen, die gewünschte Materialeigenschaft eingestellt. Dazu bedarf es entweder einer empirischen Vorgehensweise oder aber der genauen Kenntnis über das Gefüge des Materials bevor es behandelt wird, sowie darüber wie es sich während der (Wärme-)Behandlung mit der Zeit entwickelt. Von besonderem Interesse sind heutzutage solche Materialien, die sich in Zuständen weit außerhalb des thermodynamischen Gleichgewichts befinden. Einerseits lassen sich damit z. T. sehr ausgeprägte Materialeigenschaften einstellen, z. B. große mechanische Härte. Andererseits können an solchen Materialien auch prinzipielle grundlagenphysikalische Aspekte der Entwicklungsmechanismen studiert werden.

Materialien, die sehr kleine Korngrößen von unter 10 bis $20 \mathrm{~nm}$ aufweisen, werden nanokristalline Materialien (NKM) genannt. Ihr Zustand ist hochgradig gestört und gekennzeichnet durch eine hohe Defektdichte. Im Vergleich zu herkömmlichen Polykristallen sind für sie charakteristisch die sehr großen Korngrenzenflächen pro Volumeneinheit. In einem $1 \mathrm{~cm}^{3}$ großen Würfel befinden sich immerhin mehrere $100 \mathrm{~m}^{2}$ Korngrenzenfläche. Der Anteil der Atome, die zu den Korngrenzen zuzurechnen sind, kann dabei mehr als $50 \%$ betragen [GLEI89] - in Abb. 1.1 ist zur Veranschaulichung der Schnitt durch eine nanokristalline Kornstruktur schematisch dargestellt. Die Eigenschaften dieser NKM sind daher stark dominiert durch die Grenzflächen. Dies macht sie interessant als Objekt für Forschung und Technik.

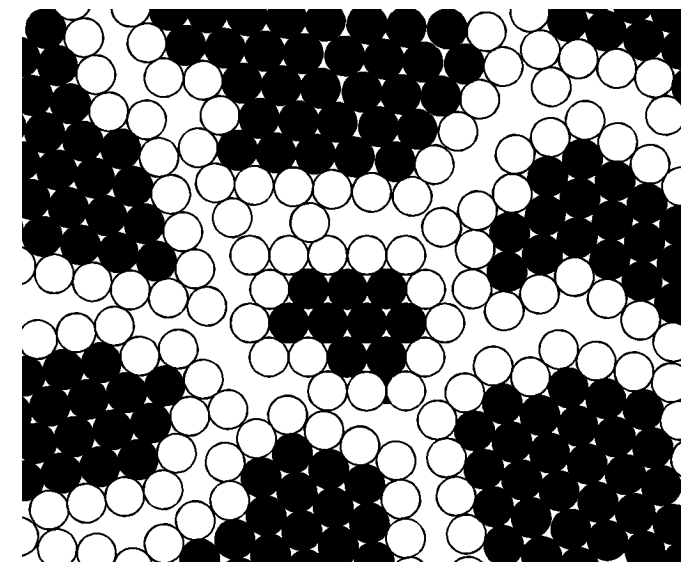

Abb. 1.1 Schematische nanokristalline Kornstruktur. Atome, die den Korngrenzen zuzurechnen sind, sind als offene Kreise dargestellt, Atome innerhalb der Körner als ausgefüllte Kreise.

Der Einfluss der Korngrenzen ist auch für nanokristalline mehrkomponentige Legierungen von großer Bedeutung. Insbesondere gilt dies bezüglich der Entwicklung der nanokristallinen Gefüge bei Wärmebehandlungen. Für das Verständnis der Zusammenhänge ist dabei die Kenntnis über die genaue Verteilung der Komponenten in den nanokristallinen Strukturen notwendig. Es liegt in der Natur der Sache, dass sich Untersuchungen diesbezüglich schwierig gestalten. Aufgrund der sehr kleinen 3dimensionalen Strukturen sind detaillierte Analysen der NKM bisher nur eingeschränkt möglich gewesen. Die moderne 3D-Atomsondentechnik [MILL96] stellt erstmals eine höchstauflösende Analytik dar, die es ermöglichen sollte die 3-dimensionale Verteilung 
der Legierungskomponenten in den Nanostrukturen zu bestimmen. Sie wurde daher für die Untersuchungen in dieser Arbeit eingesetzt.

Zwei nanokristalline binäre Legierungen wurden ausgewählt und untersucht: EisenSilber (Fe-Ag) und Nickel-Phosphor (Ni-P). Die beiden Legierungen repräsentieren Systeme mit stark unterschiedlichen chemischen Wechselwirkungen. Während einerseits Eisen und Silber eine große positive Mischungsenthalpie von ungefähr $\Delta \mathrm{H}_{\text {mix }}=+28 \mathrm{~kJ} / \mathrm{mol}$ [BOER88] aufweisen ${ }^{1}$, und damit stark repulsiv wechselwirken, verhält es sich im System Nickel-Phosphor genau umgekehrt. Die Mischungsenthalpie ist hier negativ und beträgt $\Delta \mathrm{H}_{\text {mix }}=-44 \mathrm{~kJ} / \mathrm{mol}$ [SHIM99] ${ }^{2}$. Die chemische Wechselwirkung ist also stark attraktiv.

Das Legierungs-System Eisen-Silber ist, bedingt durch die genannte repulsive chemische Wechselwirkung, durch sehr extreme Eigenschaften gekennzeichnet. Die beiden Komponenten Eisen und Silber sind im thermodynamischen Gleichgewicht selbst im flüssigen Zustand nicht mischbar. Die Randlöslichkeiten im festen Zustand sind verschwindend gering. Auf der Fe-reichen Seite beträgt sie maximal 0,022 At.\% $\mathrm{Ag}$ (in $\gamma$-Eisen) [WRIE73, MASS90]. Umgekehrt wird für die Löslichkeit von Eisen in Silber 0,007 At.\% Fe als die oberste Grenze angegeben [BERN69, MASS90]. Mit Hilfe der gepulsten Laserdepositon (PLD) lassen sich dünne Schichten nanokristalliner FeAg-Legierungen herstellen [KREB93]. Für diese Herstellungsmethode sind die relativ hohen kinetischen Energien der Teilchen bei der Deposition von ungefähr $100 \mathrm{eV}$ charakteristisch [FÄHL98]. Sie haben wesentlichen Einfluss auf die Eigenschaften der resultierenden Schichten. Röntgenographische Untersuchungen an PLD-Schichten von Störmer und Krebs [STÖR95] liefern Grund zu der Annahme, dass Silber in Eisen bis zu Konzentrationen von 14 At.\% übersättigt werden kann, da bis zu diesen Konzentrationen in den Diffraktogrammen ausschliesslich Legierungspeaks beobachtet wurden. Ähnliche Ergebnisse werden auch von Ni-Ag-Legierungen berichtet [INGE94, STÖR95]. Die Untersuchungen in dieser Arbeit befassen sich mit den Eisen-reichen mittels PLD hergestellten Fe-Ag-Legierungen. Diese Legierungen stellen extreme Nicht-Gleichgewichtszustände dar. Die treibenden Kräfte für die Entmischung in die Reinkomponenten sind in diesen Schichten sehr groß. Die Fe-Ag-Legierungen repräsentieren daher in besonderer Weise Materialien im Zustand weit weg vom thermodynamischen Gleichgewicht. Ihre Untersuchung ist daher von grundlagenphysikalischem Interesse. Der Grad der Homogenität im ,wie hergestellt“-Zustand, die Mechanismen der Entmischung und die Entwicklung der Nanostruktur dieser „Zwangslegierten“ NKM bei Wärmebehandlung sind bisher so gut wie unbekannt.

Ni-P-Legierungen können konventionell mittels außenstromloser chemischer Abscheidung hergestellt werden. Sie werden der Vereinfachung halber meist nur kurz „,chemisch Nickel“ genannt. Ihre Strukturen sind nanokristallin bei P-Gehalten bis ca. 15 At.\%. Oberhalb von 16 At.\% zeigen sie amorphe Struktur [KREY86, LEE91]. In dieser Arbeit wurden daher Legierungen mit niedrigen P-Gehalten zwischen 3 und 12 At.\% hergestellt und untersucht. Neben der chemischen Abscheidung besteht auch die Möglichkeit Ni-P-Legierungen mittels elektrolytischer Abscheidung herzustellen. Diese sind in gleicher Art nanokristallin mit vergleichbaren Korngrößen, wie auch die chemisch abgeschiedenen Legierungen [ALLE82]. Die Ergebnisse anderer Autoren, die auf Untersuchungen an elektrolytisch abgeschiedenen Ni-P-Legierungen beruhen,

\footnotetext{
${ }^{1}$ Dieser Wert gilt für eine 50 At.\%-ige Ag-Lösung in bcc-Eisen.

${ }^{2}$ Dieser Wert gilt für eine 50 At.\%-ige P-Lösung in fcc-Nickel.
} 
wurden daher in dieser Arbeit parallel zu Untersuchungen an chemisch abgeschiedenem Nickel-Phosphor diskutiert.

Das Legierungssystem Nickel-Phosphor ist relativ komplex. In Abb. 1.2 ist das Gleichgewichts-Phasendiagramm nach [LEE91] abgebildet. Es gibt insgesamt 11 geordneten Phasen. Die bereits erwähnte große attraktive chemische Wechselwirkung erklärt die Existenz der vielen geordneten Phasen. Die erste bei niedrigen P-Gehalten ist die $\mathrm{Ni}_{3} \mathrm{P}$-Phase. In dem Bereich zwischen 0 und 25 At.\% P befindet sich ein Eutektikum bei 19 At.\% P. Die Löslichkeit von Phosphor in der fcc-Nickel-Phase ist sehr gering. Es sind maximal 0,32 At.\% bei der eutektischen Temperatur von $870{ }^{\circ} \mathrm{C}$. Die Legierungen in dem Konzentrationsbereich wie sie hier untersucht wurden (3-12 At.\% P, s. o.) würden also im thermodynamischen Gleichgewicht in der fcc-Nickel- und in der $\mathrm{Ni}_{3} \mathrm{P}-$ Phase mit dem Hebelgesetz folgenden Volumenanteilen vorliegen.

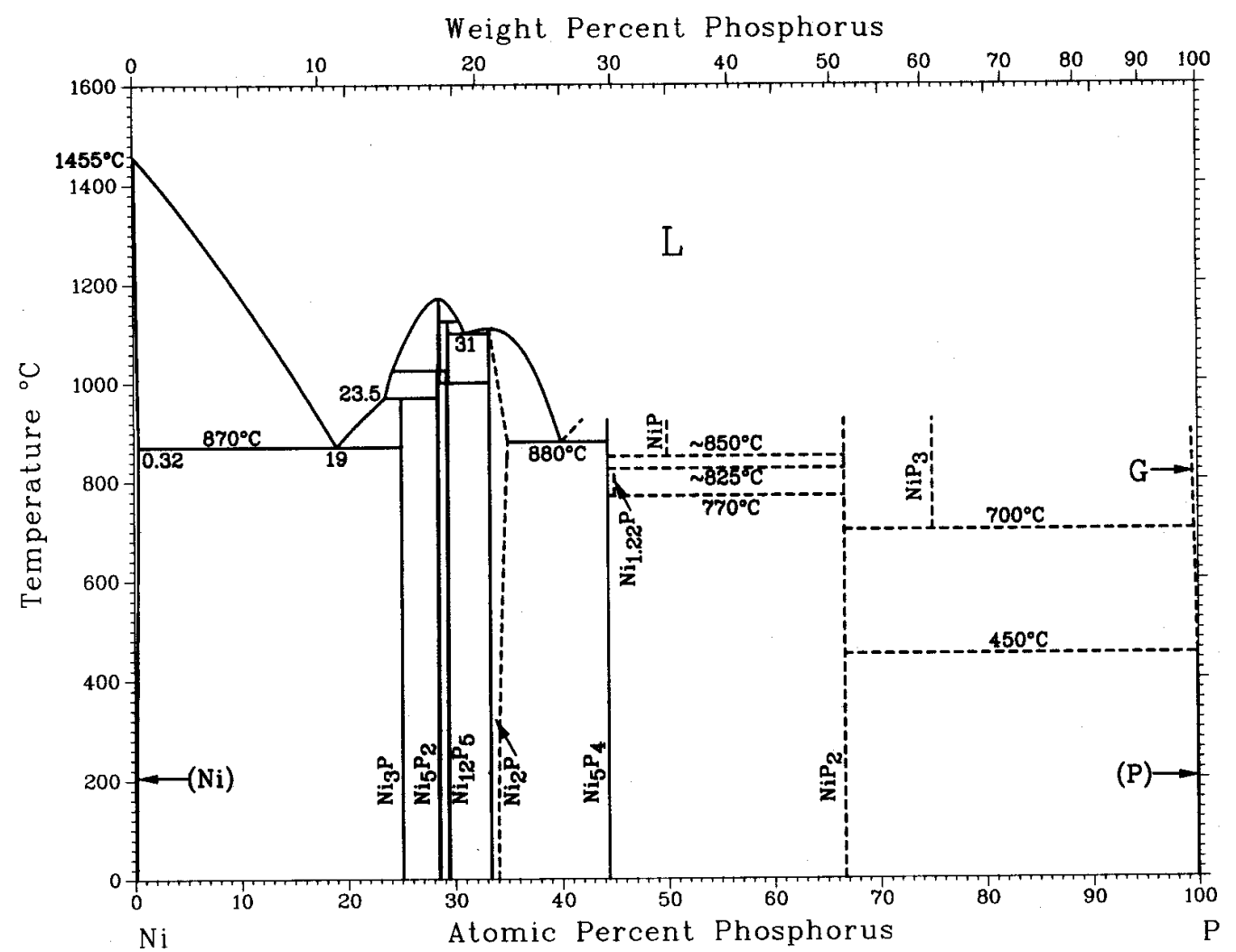

Abb. 1.2 Phasendiagramm des Systems Nickel-Phosphor nach [LEE91].

Ni-P-Legierungen sind bereits seit Jahrzehnten im technischen Einsatz zur Lösung von Korrosions- und Verschleißproblemen, zur Beschichtung von Nichtleitern, bei der Leiterplatten-Herstellung und anderen Anwendungen [RIED89]. Allein in Europa werden jährlich mehrere hundert Tonnen des Materials verbraucht [RIED91]. Trotz der langjährigen Verwendung als Werkstoff sind jedoch insbesondere bei den nanokristallinen Ni-P-Legierungen noch viele Fragen, die detaillierte Mikrostruktur betreffend, offen. Es wurden interessante Eigenschaften beobachtet, über deren Ursache noch wenig bekannt ist, und die z. T. kontrovers diskutiert werden. So wird bei Auslagerungen eine thermische Stabilität der Kornstruktur beobachtet [BOYL91, MEHT95, ZHAN98]. Das nanokristalline Gefüge bleibt nach anfänglichen geringfügigen Veränderungen bis zu Temperaturen von $320^{\circ} \mathrm{C}$ und mehr stabil. In der Literatur werden sehr unterschiedliche mögliche Ursachen dafür diskutiert: Behinderung des Kornwachstums durch $\mathrm{Ni}_{3} \mathrm{P}-$ 
Ausscheidungen (Zener-Effekt) und durch Tripelpunkte, sowie ein Solute-Drag-Effekt durch homogen gelöste P-Atome. Ein großes Problem bei der bisherigen Diskussion ist aber, dass nur wenige bzw. keine Daten über die (räumliche) Verteilung des Phosphors in den Legierungen vorliegen. Die Untersuchungen in dieser Arbeit sollen auch dazu beitragen diese Lücke zu schließen.

Die vorangegangenen Ausführungen verdeutlichen, dass detaillierte Untersuchungen der Nanostrukturen von nanokristallinen Fe-Ag- und Ni-P-Legierungen notwendig sind und dazu beitragen können, die Eigenschaften der Materialien nach deren Herstellung und nach Wärmebehandlungen besser zu verstehen. Im Rahmen dieser Arbeit sollten daher ausgewählte Fe-Ag- und Ni-P-Legierungen hergestellt und anschließend die Zustände der Materialien nach ihrer Herstellung und deren Entwicklung bei Wärmebehandlungen nanostrukturell charakterisiert werden. Den Schwerpunkt bildeten dabei die Untersuchungen mit der Atomsonden-Technik, insbesondere der Tomographischen Atomsonde (TAP). Diese Analyse-Methoden werden in Kap. 2.4 ausführlicher beschrieben. Dabei wird einerseits auf die Mess-Technik und andererseits auch auf die Auswerteverfahren eingegangen.

Die Herstellung der Fe-Ag-Proben ist im Kapitel 2.1 dargestellt. Es wurden Schichten auf Spitzen speziell für die Analysen mit der TAP präpariert. Die Untersuchungen an den Fe-Ag-Legierungen auf TAP-Spitzen werden in Kapitel 3 vorgestellt. Parallel dazu wurden weitere Untersuchungen an planaren Schichten in einer Arbeit von Störmer durchgeführt [STÖR98]. Es konnten so die Ergebnisse beider Untersuchungen unmittelbar gegenübergestellt werden. In Kap. 3.5 werden die Ergebnisse der Untersuchungen an Fe-Ag-Schichten auf Spitzen diskutiert.

Die Herstellung der Ni-P-Legierungen und Präparation der Proben sind in den Kapiteln 2.2 und 2.3 nachzulesen. Die Untersuchungen zu diesem System werden in Kapitel 4 dargestellt. Dabei werden zunächst die Zustände der Legierungen im Abscheidezustand nach der Herstellung behandelt. Anschließend wird genauer auf die Entwicklung der Mikro- und Nanostruktur bei Wärmebehandlungen eingegangen. In Kap. 4.3 erfolgt die Diskussion der Ergebnisse. Es werden die Strukturen und ihre Entwicklung diskutiert. Außerdem wird gesondert auf die thermische Stabilität der Ni-P-Legierungen und ihre möglichen Ursachen eingegangen. 


\section{Experimente}

Im Rahmen dieser Arbeit wurden Fe-Ag- und Ni-P-Legierungen hergestellt und untersucht. Dabei wurden unterschiedliche Analysemethoden angewendet und auch Simulationen durchgeführt. Entsprechend der vorliegenden Nanostrukturen der Materialien wurde für die Analyse mit höchster Auflösung die Atomsondentechnik mit konventionellem Detektor (AP) und mit neuen ortsauflösenden Detektoren (3DAP) eingesetzt.

Die Fe-Ag-Legierungen wurden einerseits als dünne Schichten auf FIM-Substratspitzen aufgebracht und anschließend im Transmissions-Elektronenmikroskop (TEM) und mit der AP oder der TAP untersucht. Andererseits wurden parallel dazu Schichten auf planaren Substraten deponiert. Die Untersuchungen an diesen planaren Proben $-\mathrm{u}$. a. mit TEM, Röntgendiffraktometrie (XRD) und energiedispersiver Röntgenanalyse (EDX), sowie Schichtdickenmessungen - wurden von M. Störmer und K. Sturm durchgeführt [STÖR98, STUR00].

Die Ni-P-Legierungen wurden im Rahmen dieser Arbeit mit der TAP sowie mit anderen Analysemethoden charakterisiert. Es sind $\mathrm{zu}$ nennen die Differential-ScanningCalorimetry (DSC), die XRD, das TEM und die Elektronenstrahlmikrosonde (EMS).

\subsection{Herstellung der Fe-Ag-Proben}

Für die Herstellung der Fe-Ag-Legierungen wurde die gepulste Laserdeposition (PLD) verwendet [CHRI94]. Die PLD ist mittlerweile eine verbreitete Methode zur Herstellung dünner Schichten, mit deren Hilfe sich auch unterschiedlichste metallische Schichten herstellen lassen [GAVI90, CHRI94, KREB97, FÄHL98]. Bei der PLD wird ein gepulster energiereicher Laserstrahl auf ein Target fokussiert. Dadurch wird das Targetmaterial kurzfristig stark erhitzt und in einem komplexen Prozess, der sog. Ablation, abgetragen [DYER89]. Es bildet sich ein senkrecht zur Targetoberfläche expandierendes Plasma, welches sich auf dem gegenüberliegenden Substrat niederschlägt.

Die Fe-Ag-Legierungen für diese Arbeit wurden mit Hilfe eines Krypton-Fluorid-Lasers der Firma Lambda-Physik, Excimerlaser LPX 110i, hergestellt. Dabei wurde mit $30 \mathrm{~ns}$ langen UV-Pulsen mit einer Wellenlänge von $248 \mathrm{~nm}$ und einer Repititionsrate von 10 $\mathrm{Hz}$ gearbeitet. Die Laserenergie pro Puls betrug etwa 100 bis $115 \mathrm{~mJ}$, was einer Energiedichte von ca. 6-8 J/cm² entsprach. Die Beschichtungen erfolgten unter Ultrahochvakuum (UHV) -Bedingungen bei einem Restgasdruck von etwa $10^{-8} \mathrm{hPa}$. Als Targets wurden hochreine Fe- und Ag-Bleche verwendet, die auf einem Targethalter fixiert wurden, der mit Hilfe eines Schrittmotors auf und ab bewegt werden konnte. Für die Herstellung der Legierungsschichten wurden dann die Bleche abwechselnd für die Dauer weniger Pulse in den Strahlengang gebracht. Dieses Verfahren wird auch bei planaren Schichten standardmäßig angewendet [FÄHL98, STÖR98]. Unterschiedliche Ag-Konzentrationen wurden eingestellt über das Verhältnis der Pulszahlen mit denen im Wechsel auf die beiden Targets geschossen wurde. Auf ein einzelnes Target wurde nicht mehr als 15 mal vor einem Wechsel auf das andere Target geschossen, was in etwa der Deposition einer halben Monolage $(0,1 \mathrm{~nm})$ entspricht. Da die Targets einer gewissen Alterung - ein bekannter Effekt bei der PLD [KREB93, FÄHL98] - unterlagen, also die Depositionsrate mit der Zeit abnahm, mussten die Pulszahlverhältnisse und die Gesamtzahl an Pulsen an jedem Versuchstag neu festgelegt werden. Dadurch konnten definierte Ag-Konzentrationen nur in gewissen Grenzen reproduziert werden. 
Die PLD ist eine Dünnschichtmethode. Typische Schichtdicken betragen wenige 100 $\mathrm{nm}$. Eine Präparation von Spitzen aus dem zu untersuchenden Material - wie bei den freitragenden, verhältnismäßig dicken Ni-P-Schichten - war daher nicht möglich. Für die Atomsonden-Untersuchungen wurden deshalb die Fe-Ag-Schichten direkt auf zuvor präparierte Wolfram-Spitzen aufgebracht. Diese wurden aus Wolfram-Draht hergestellt, der auf konventionelle Art mit einem Elektrolyten aus wässriger 1-molarer $\mathrm{NaOH}$ Lösung bei einer Spannung von 5 V (AC) elektrolytisch gedünnt wurde [MILL96]. Die Wolfram-Spitzen wurden dann in einer Atomsonde im Feldionenmikroskop-(FIM)Modus, s. Kap. 2.4, durch Feldverdampfung entwickelt bis sie ein vollständig ausgeprägtes FIM-Bild zeigten. D. h. die Spitzen wurden so weit abgerundet, dass ihre Oberfläche am Apex halbsphärenförmig und absolut frei von Schmutz oder Elektrolytresten war. Die so gereinigten Wolfram-Spitzen wurden daraufhin zur Kontrolle im TEM untersucht und anschließend in die Laserkammer in einen speziell konstruierten, rotierbaren Halter eingebaut. Dieser ermöglichte den Einbau von gleichzeitig bis zu 6 Spitzen, die kreisförmig in einem Durchmesser von $2 \mathrm{~cm}$ angeordnet waren und in deren Mitte zusätzlich ein planares Silizium-Substrat auf Höhe der Spitzenapexes fixiert werden konnte, so dass bei der Beschichtung der Spitzen gleichzeitig immer auch eine planare Referenzschicht unter denselben Bedingungen hergestellt wurde. Die Beschichtungen erfolgten in allen Fällen bei Raumtemperatur ohne separate Kühlung der Substrate. Eine schematische Skizze des Versuchsaufbaus ist in Abb. 2.1 dargestellt.

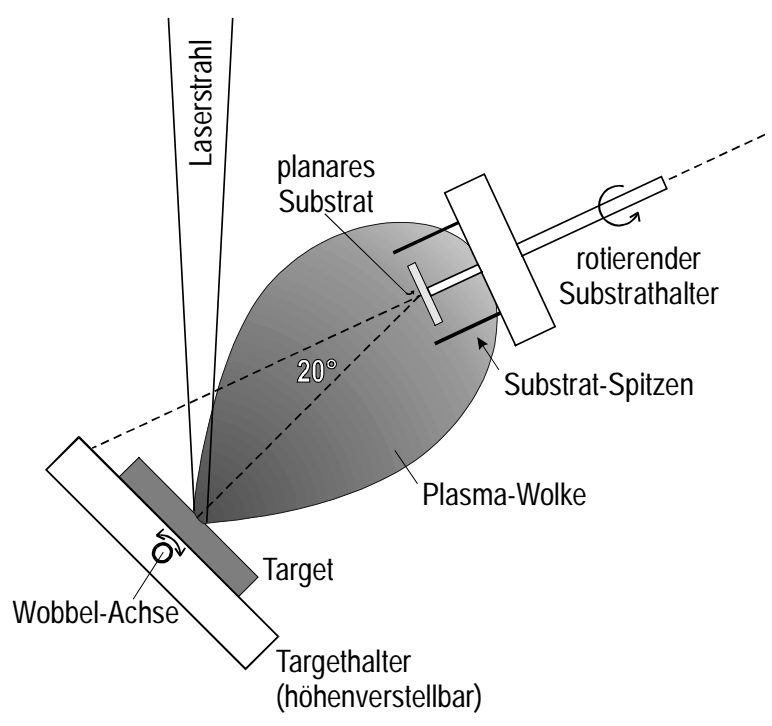

Abb. 2.1 Schematischer Aufbau der gepulsten Laserdeposition (PLD) auf AP/TAP-Spitzen. Der Abstand Target - Spitzen beträgt ca. $40 \mathrm{~mm}$.

Die Spitzen zeigten nicht senkrecht auf das Target, wurden also nicht frontal beschichtet, sondern unter einem Winkel von $20 \mathrm{Grad}$. Dadurch und durch die zusätzliche Rotation des Halters während des Herstellungsprozesses war gewährleistet, dass die Spitzen von allen Seiten gleichmäßig beschichtet wurden und dass es nicht zu einer ungünstigen Pfropfenbildung kam. Der Abstand der Spitzen zum Target betrug ca. $40 \mathrm{~mm}$. Nach erfolgreicher Beschichtung wurden die Spitzen ein weiteres mal im TEM untersucht bevor sie, sofern sie geeignet schienen, in eine Atomsonde eingebaut wurden. Parallel dazu wurden jeweils der Ag-Gehalt, mittels EDX, und die Schichtdicke, mittels eines Profilometers, der planaren Referenzschichten bestimmt. 


\subsection{Herstellung der Ni-P-Schichten}

Die im Rahmen dieser Arbeit untersuchten Ni-P-Legierungen wurden als Schichten durch außenstromloses chemisches Abscheiden hergestellt. Diese Art der Abscheidung ist ein weithin bekanntes und ein weit verbreitetes, industriell angewandtes Verfahren [RIED91]. Für die Herstellung der hier verwendeten Proben kamen deshalb kommerziell erhältliche Elektrolyt-Bäder zum Einsatz. Wesentlicher Bestandteil dieser Bäder sind $\mathrm{Ni}^{2+}$-Ionen, als Nickelsulfat, und $\mathrm{H}_{2} \mathrm{PO}_{2}{ }^{-}$-Ionen, als Natriumhypophosphit. Hinzu kommen Komplexbildner, Puffersubstanzen, Stabilisatoren und sogenannte Beschleuniger. Die genaue Zusammensetzung der Elektrolyte wird von den Herstellerfirmen aus Gründen des Wettbewerbs nicht angegeben. Die wesentliche chemische Reaktion für die Abscheidung von Nickel ist eine Redoxreaktion [RIED91]:

$$
\begin{aligned}
& \mathrm{Ni}^{2+}+2 \mathrm{e}^{-} \rightarrow \quad \mathrm{Ni} \\
& 2 \mathrm{H}_{2} \mathrm{PO}_{2}^{-}+2 \mathrm{H}_{2} \mathrm{O} \rightarrow \quad 2 \mathrm{H}_{2} \mathrm{PO}_{3}^{-}+\mathrm{H}_{2}+2 \mathrm{H}^{+}+2 \mathrm{e}^{-} .
\end{aligned}
$$

Der Phosphor hingegen wird als atomarer Phosphor in die Schichten eingebaut. Für seine Entstehung gibt es unterschiedliche Reaktionsmodelle [RIED91]. Der genaue Mechanismus der Entstehung ist bisher jedoch noch ungeklärt.

Schichten mit nominellen Konzentrationen von 3,6 At.\% P und 11,8 At.\% P wurden am Institut für Werkstofftechnik der Universität der Bundeswehr in Hamburg hergestellt, s. [KREY95, MÜLL96], und von H. Kreye freundlicherweise zur Verfügung gestellt ${ }^{3}$. Als Substrate dienten polierte Titan-Bleche, von denen sich die nicht sehr fest haftenden Schichten leicht abziehen ließen. Schichten mit 5,9 At.\% P und 6,4 At.\% P wurden in Zusammenarbeit mit der Firma Enthone-OMI (Deutschland) $\mathrm{GmbH}$ in Solingen hergestellt. Als Substrate dienten $6 \times 10 \mathrm{~cm}^{2}$ große Aluminium-Bleche, die einseitig mit Klebestreifen abgeklebt und vor der Beschichtung gereinigt und mit einer Zinkatbeize behandelt wurden, um das anfängliche Wachstum und die Haftung der Schichten zu erleichtern. Als Elektrolyt-Bad wurde der Typ ENPLATE Ni 429 E von der Firma Enthone-OMI verwendet. Die Abscheidung erfolgte in einem 51-Becherglas auf einer Heizplatte, in dem der Elektrolyt ständig gerührt wurde. Die Temperatur betrug 87 bzw. $88{ }^{\circ} \mathrm{C}$. Der pH-Wert wurde auf 6,9 bzw. 7,0 eingestellt, regelmäßig kontrolliert und ggf. durch Hinzufügen von Ammoniumhydroxidlösung auf dem Sollwert gehalten. Der Abscheidevorgang dauerte 2,5 bzw. 2,2 h. Die Schichten hatten am Ende eine Dicke von 58 bzw. $35 \mu \mathrm{m}$, was einer Abscheiderate von 23,2 bzw. 15,9 $\mu \mathrm{m} / \mathrm{h}$ entspricht. Laut Herstellerangaben ist dieser Elektrolyttyp geeignet für die Abscheidung von Ni-PSchichten mit P-Gehalten von 2-4 Gew.\% (entsprechend 3,7-7,3 At.\%). Die für diesen Badtyp gewählten relativ hohen Temperaturen und $\mathrm{pH}-$ Werte ließen einen eher geringeren P-Gehalt erwarten, als der hergestellte. Die Ursache hierfür ist unklar. Die fertigen Schichten wurden schließlich für die weitere Verwendung als Folien von den Substraten abgelöst, indem die Al-Bleche in verdünnter Natronlauge (100 g NaOH auf 1 Liter Wasser) aufgelöst wurden.

\subsection{Präparation der Ni-P-Proben}

Die Ni-P-Folien sind relativ leicht zerbrechlich. Ihre Sprödigkeit nimmt offensichtlich mit dem P-Gehalt zu. Das Abtrennen von Probenstücken erfolgte daher mit Hilfe einer

\footnotetext{
${ }^{3}$ Die hier genannten nominellen Konzentrationen beziehen sich auf die eigenen Messungen, s. Kap. 4.1.1. Kreye et al. geben für die Proben P-Gehalte von 3,2 bzw. 10,4 At.\% an.
} 
aufschlagenden Schere, die über die gesamte Schnittkannte gleichmäßig viel Kraft ausübt. Es wurde eine handelsübliche Rosenschere verwendet, deren untere Auflagefläche austauschbar und nachpolierbar war.

Für die EMS-Analysen wurden Probenstücke mit Hilfe einer Einbett-Vorrichtung (Prestopress-2 der Firma Struers) bei ca. $100{ }^{\circ} \mathrm{C}$ in graphithaltigen, und damit leitfähigen Acrylharz eingebettet und anschließend im Querschliff mechanisch poliert.

Für die TEM-Untersuchungen wurden die für die AP bzw. TAP präparierten Spitzen verwendet. AP/TAP-Spitzen haben im vorderen Bereich eine typische Dicke von 20 bis $100 \mathrm{~nm}$ und sind somit meistens gut durchstrahlbar. Darüberhinaus sind auch TEMProben auf konventionelle Art hergestellt worden. Dazu wurden aus den Folienstücken kreisrunde Scheibchen mit einem Durchmesser von $3 \mathrm{~mm}$ ausgestanzt und mit Hilfe einer Ionendünnungsanlage Duomill 600 der Firma Gatan gedünnt. Die Proben wurden dabei auf eine Temperatur von $77 \mathrm{~K}$ gekühlt und beidseitig unter einem Winkel von je 17,5 Grad mit Ar-Ionen beschossen. Die Beschleunigungsspannung betrug $5 \mathrm{kV}$ und der Ionenstrom $1 \mathrm{~mA}$.

Die Präparation der AP/TAP-Spitzen begann jeweils mit dem Herausschneiden kleiner ca. $4 \mathrm{~mm}$ langer und ca. 0,5 mm breiter Stäbchen aus den Ni-P-Folien. Diese Stäbchen wurden in Plexiglashalter eingeklemmt und zunächst einseitig mechanisch poliert. Es wurde Nassschleifpapier der Körnungen 1200, 2400 und 4000 verwendet. Danach wurden die Proben um 180 Grad gedreht, erneut eingeklemmt und mechanisch poliert bis das Stäbchen über seine Länge hinweg einen möglichst quadratischen Querschnitt von ca. $40 \times 40 \mu \mathrm{m}^{2}$ aufwies. Diese Rohlinge wurden ggf. wärmebehandelt und dann in Metallröhrchen eingeklemmt, die für den späteren Einbau der Spitzen in die jeweilige TAP geeignet waren. An der Sonde in Rouen wurden Nickelröhrchen verwendet, an der Göttinger Sonde Kupferröhrchen. Die weitere Präparation bestand in einem elektrolytischen Polieren. Die verwendeten Parameter sind in Tab. 2.1 aufgeführt. Es wurde die Mikropoliertechnik [MILL96] verwendet für die eine entsprechende Halte- und Manipuliervorrichtung gebaut wurde. Bei dieser Technik wird eine ringförmige Gold-Elektrode mit einem Elektrolyttropfen versehen. Die zu präparierende Spitze wird dann unter Beobachtung durch ein optisches Auflichtmikroskop mit ca. 50-facher Vergrößerung in dem Tropfen gedünnt. Der Vorteil dieser Methode ist, dass auch sehr kurze Spitzen poliert werden können, da die Form des Tropfens konvex ist und somit die Spitze an der Grenzfläche zwischen Elektrolyt und Luft durch das Mikroskop gut beobachtet werden kann.

\begin{tabular}{|l|l|}
\hline Elektrolyt & $\begin{array}{l}\text { Perchlorsäure }(60 \% \text {-ig) }+ \text { 2-Butoxyethanol, Verhältnis 1:9, } \\
\text { frisch angesetzt. }\end{array}$ \\
\hline Temperatur & $\begin{array}{l}\text { ca. } 5{ }^{\circ} \mathrm{C}, \text { vor jeder Präparation wurde ein neuer Tropfen aus der } \\
\text { Vorratsflasche im Kühlschrank entnommen }\end{array}$ \\
\hline Spannung & $+22 \mathrm{~V}(\mathrm{DC})$ zu Beginn, $+16 \mathrm{~V}$ (DC) zum Schluss \\
\hline Technik & Mikropoliertechnik mit Elektrolyttropfen \\
\hline
\end{tabular}

Tab. 2.1 Parameter für das elektrolytische Polieren der Ni-P-TAP-Spitzen.

Die Wärmebehandlungen der Ni-P-Proben wurden in einer DSC-Apparatur, einer DSC7 der Firma Perkin-Elmer, vorgenommen. Die Proben wurden dabei kontinuierlich mit Argon-Gas umspült, um Einflüsse durch Luftsauerstoff zu minimieren. Da die 
thermischen Analysen mit demselben Gerät durchgeführt wurden, war sichergestellt, dass die TEM bzw. die TAP-Proben sich in einem wohldefinierten Auslagerungszustand befanden.

\subsection{Konventionelle und Tomographische Atomsonde}

Für die Untersuchung der Nanostruktur der Fe-Ag- und der Ni-P-Proben auf feinster Skala wurde die Atomsondentechnik in unterschiedlichen Ausführungen verwendet (konventionelle Atomsonde und Tomographische Atomsonde). Moderne Atomsonden ermöglichen die chemische Analyse von kleinsten Probenvolumina auf einer nm-Skala. Die Zusammensetzung in einem Volumen kann durch einfaches Zählen von Atomen einer jeden Sorte bestimmt werden. Die Atomsondentechnik bietet damit die höchste Auflösung für chemische Analysen in allen drei Raumdimensionen, die derzeit möglich ist. Die Funktionsweise von Atomsonden ist in der Literatur bereits umfangreich beschrieben worden [MÜLL69, BOWK70, MILL89, MILL96]. Im folgenden sollen daher nur die wesentlichen Grundprinzipien der Technik im allgemeinen sowie die wichtigen Besonderheiten der konkret verwendeten Apparaturen dargestellt werden.

\subsubsection{Atomsondentechnik}

Der Begriff Atomsonde, im heutigen Sinne, bezeichnet eine Analytik, die auf Flugzeitmassenspektrometrie einzelner Atome basiert. Atomsonden sind immer kombiniert mit einer abbildenden Methode, dem Feldionenmikroskop (FIM). Eine Atomsonde kann durch einfaches Umschalten entweder im abbildenden FIM-Modus oder im analytischen Atomsonden-Modus betrieben werden. Das Grundprinzip für die Funktion ist bestechend simpel: Das Material wird zunächst in Form einer Spitze, die einen vorderen Krümmungsradius $r_{t}$ von ca. 15 bis $50 \mathrm{~nm}$ aufweisen muss, präpariert, s. a. Kap. 2.1 und 2.3. Nur elektrisch leitfähige Materialien lassen sich analysieren. An die Spitze wird dann, unter Ultrahochvakuumbedingungen (Restgasdruck ca. $10^{-9} \mathrm{hPa}$ ) und bei Kühlung auf Temperaturen von 20 bis $100 \mathrm{~K}$, eine elektrische Hochspannung $\mathrm{U}_{\mathrm{DC}}$ von einigen Kilovolt angelegt. Bedingt durch den kleinen Krümmungsradius $r_{t}$ entstehen an der Spitze hohe elektrische Felder mit Feldstärken F von mehr als $50 \mathrm{~V} / \mathrm{nm}$. Es gilt

$$
F=\frac{U_{D C}}{k \cdot r_{t}}
$$

wobei k = 4-9 der Geometriefaktor ist [MÜLL69]. Diese hohen Feldstärken werden unterschiedlich genutzt für den Betrieb im FIM-Modus und im Atomsonden-Modus.

Bei der Feldionenmikroskopie wird der Spitze im Abstand von wenigen Zentimetern ein Bildschirm, bestehend aus einem Ionenverstärker und einem mit Phosphor beschichteten Leuchtschirm gegenübergestellt. Außerdem werden Bildgase, typischerweise Neon, Helium oder Wasserstoff, in die Vakuumkammer eingelassen mit Partialdrücken von etwa $10^{-5} \mathrm{hPa}$. Die Bildgasatome werden im hohen elektrischen Feld vor der Spitze ionisiert und zum Bildschirm hin beschleunigt, wo sie einen Leuchtpunkt erzeugen. Hervorgerufen durch die atomare Struktur der Spitzenoberfäche und die unterschiedliche chemische Natur von verschiedenen Spitzenatomen variiert die Ionisationswahrscheinlichkeit entlang der Oberfläche. Durch den kontinuierlichen Bildgasionenstrom entsteht dadurch auf dem Bildschirm ein Bild der Oberfläche mit bis zu atomarer Auflösung. Die Abbildung entspricht näherungsweise einer Zentralprojektion mit einer Vergrößerung M von 


$$
M=\frac{R}{b \cdot r_{t}}
$$

wobei $\mathrm{b}$ der Bildkompressionsfaktor, $\mathrm{R}$ der Abstand Spitze zu Bildschirm und $\mathrm{r}_{\mathrm{t}}$ der Spitzenradius ist [MÜLL69]. Der Faktor b berücksichtigt die Abweichung von der Zentralprojektion. Er beträgt zwischen 1,5 und 1,8 [MǗLL69]. Es ergeben sich so typische Vergrößerungen von $10^{5}$ bis $10^{7}$.

Im Atomsonden-Modus wird der Spitze ein Detektor in $\mathrm{L}=0,5$ bis $1 \mathrm{~m}$ Entfernung gegenübergestellt, der das Auftreffen einzelner Ionen registrieren kann. Zusätzlich zu der Grundspannung $U_{D C}$ wird dann ein kurzer, wenige Nanosekunden dauernder, kleiner Hochspannungspuls der Höhe $U_{P}$ an die Spitze angelegt, so dass die sogenannte Feldverdampfungsfeldstärke $F_{f}$ überschritten wird. Dadurch werden einzelne Atome der Spitzenoberfläche feldverdampft [MÜLL89, MILL96], d. h. ionisiert und im elektrischen Feld zum Detektor hin beschleunigt. Durch Messung der Flugzeit t kann aufgrund der bekannten kinetischen Energie der Ionen die spezifische Masse bestimmt werden. Es ist

$$
\frac{m}{n}=\frac{2 e \cdot\left(U_{D C}+U_{P}\right) \cdot t^{2}}{L^{2}},
$$

wobei $\mathrm{n}$ der Ionisationsgrad ist. Die Atomssonde ist also, wie bereits erwähnt, im wesentlichen ein Flugzeitmassenspektrometer. Durch kontinuierliches Pulsen können die Atome von der Spitzenoberfläche und durch sukzessives Abtragen auch ins Volumen hinein analysiert werden.

Je nach Art des Detektors unterscheidet man konventionelle Atomsonden (AP), die lediglich die Flugzeit erfassen können, sowie ortsauflösende 3D-Atomsonden (3DAP), die neben der Flugzeit auch den Auftreffpunkt der einzelnen Ionen aufzeichnen. Bei konventionellen Atomsonden wird durch eine Aperturblende, die i. d. R. als Loch im FIM-Bildschirm realisiert ist, die laterale Auflösung festgelegt. Sie beträgt auf die Spitzenoberfläche projiziert ca. 2 bis $5 \mathrm{~nm}$. Die Tiefenauflösung hingegen ist bestimmt durch das sukzessive Abtragen der Atomlagen von der Spitzenoberfläche. Mit konventioneller Atomsonde erhält man im Ergebnis Konzentrationsanalysen mit etwa 0,2 nm Tiefenauflösung und einer lateralen Auflösung von 2 bis $5 \mathrm{~nm}$ [MILL96].

Das Herzstück von 3D-Atomsonden bildet der positionsempfindliche Detektor. Die Tomographischen Atomsonden (TAP), die in dieser Arbeit verwendet wurden, besitzen einen Detektor, der im wesentlichen aus einem Elektronenvervielfacher, bestehend aus zwei hintereinandergeschalteten Mikrokanalplatten (MCP), einem Array von 96 Elektroden und einer Hochleistungs-Auswerteelektronik besteht. Der schematische Aufbau einer TAP ist in Abb. 2.2 dargestellt.

Durch das Auftreffen eines Ions auf die erste MCP wird eine Elektronen-Kaskade ausgelöst. Dieses Signal wird aufgezeichnet und für die Flugzeitmessung verwendet. Die Elektronen-Kaskade wird durch die zweite MCP weiter verstärkt. Es entsteht so hinter den MCPs eine Elektronen-Wolke, die auf dem dahinter befindlichen $10 \times 10 \mathrm{~cm}^{2}$ großen Elektrodenarray deponiert wird. Der Elektronen-Spot überstreicht i. d. R. mehrere Elektroden. Die auf jeder Elektrode deponierte Ladung wird durch schnelle AD-Wandler-Elektronik gemessen. Dadurch kann der Schwerpunkt der Elektronenladung, und damit die Position des aufgetroffenen Ions auf dem Detektor bestimmt werden [BLAV93]. Sofern die Elektronen-Spots sich nicht überlappen, können auch mehrere Ionen, die gleichzeitig auf den Detektor treffen, positioniert werden. Außerdem 
verfügt der Detektor über mehrere (5 bzw. 8) schnelle AD-Wandler-Elektroniken, so dass ggf. auch mehrere Ionen mit unterschiedlichen spezifischen Massen und entsprechend unterschiedlichen Flugzeiten detektiert werden können.

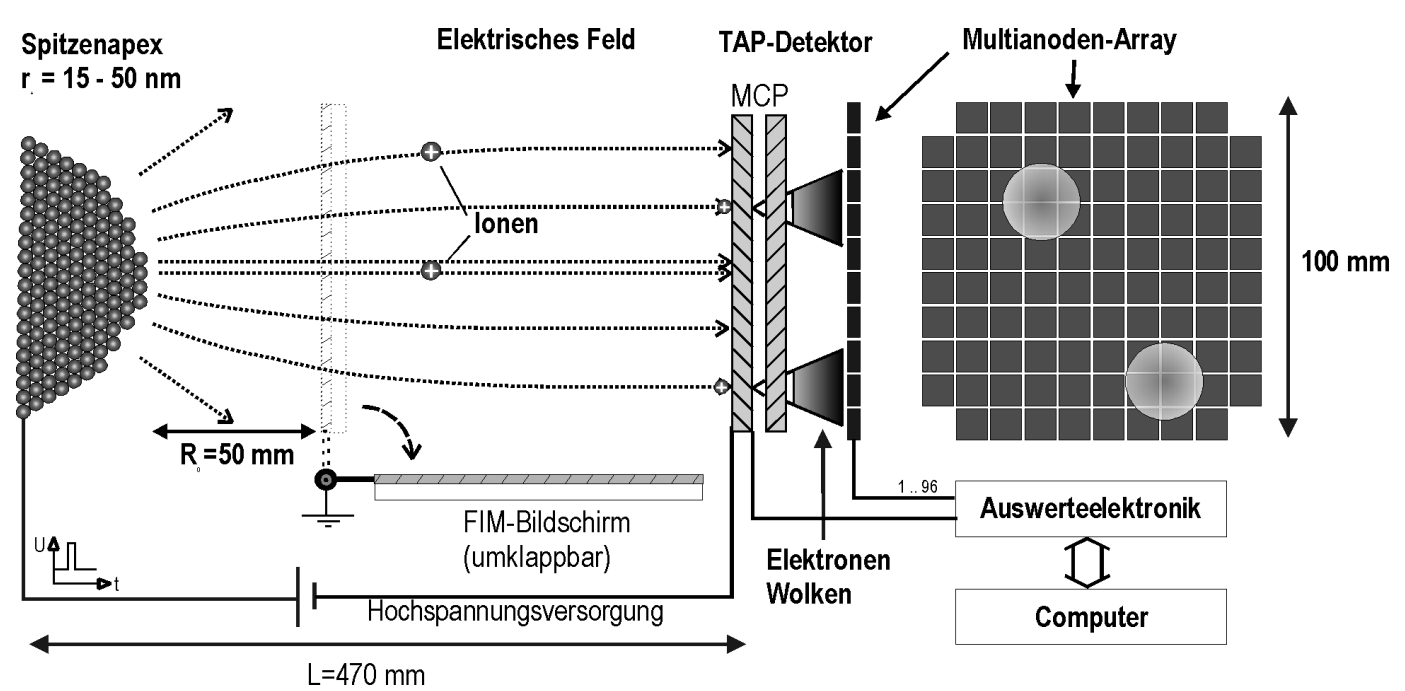

Abb. 2.2 Die Tomographische Atomsonde (TAP).

Die gewonnenen Daten (spezifische Massen und Positionen auf dem Detektor) ermöglichen schließlich die 3-dimensionale (3D) Rekonstruktion der ursprünglichen Positonen der Atome im Probenvolumen. Sie erfolgt im nachhinein mit Hilfe spezieller Auswertesoftware, s. a. Kap. 2.4.2, auf einer Workstation. Der 3D-Rekonstruktion liegen die fundamentalen Gleichungen 2.1 und 2.2 zugrunde. Die Tiefenskalierung erfolgt dabei auf Basis der Anzahl detektierter Ionen und der Atomdichte [DECO94, BAS 95, BAS-97]. Das rekonstruierte Probenvolumen steht dann für weitere Auswertungen und Analysen mit geeigneten Software-Tools zur Verfügung. Die räumliche Auflösung der TAP beträgt im Idealfall ca. 0,2 $\mathrm{nm}$ in Messrichtung [BLAV93] und, bedingt durch den Prozess der Feldverdampfung, ca. 0,5 nm lateral [MILL96]. Bei heterogenen Materialien kann sie aber auch schlechter sein, insbesondere bei unterschiedlichen lokalen Feldverdampfungsfeldstärken und den dadurch hervorgerufenen lokalen Vergrößerungseffekten [BLAV96, MILL96].

\subsubsection{Mess- und Daten-Auswertesoftware}

Die konventionelle Göttinger Atomsonde (AP) wie sie in [GEBE92] beschrieben wurde ermöglichte das Pulsen mit einer Frequenz von $10 \mathrm{~Hz}$. Durch die Integration eines moderneren schnellen Halbleiterpulsers von der Firma Kindbrisk Ltd., Großbritannien, waren Pulsfrequenzen von bis zu $1000 \mathrm{~Hz}$ möglich geworden. Die vorhandene PCunterstützte Atomsondensteuerung [GEBE94] musste daher im Rahmen dieser Arbeit neu konzipiert werden. Insbesondere wurde ein schneller Datenbus zwischen dem Computer und der als CAMAC ${ }^{4}$-Modul vorhandenen Uhr und dem Analog-DigitalWandler integriert sowie eine neue Messsoftware entwickelt. Das in Turbo-Pascal geschriebene Messprogramm ermöglichte auch die automatische Steuerung von Messungen, so dass mit einer möglichst konstanten Feldverdampfungsrate gearbeitet werden konnte. Für die anschließende Datenauswertung wurde die vorhandene oder Standardsoftware verwendet.

\footnotetext{
${ }^{4}$ Computer Automated Measurement and Control, IEEE-583
} 
Bei AP-Analysen müssen die wesentlichen Parameter für eine Analyse (Ort und Größe der Apertur) bereits zum Zeitpunkt der Messung festgelegt werden. Bei der Auswertung können dann lediglich noch Blocklängen definiert werden, um Konzentrationen und Tiefenskalen zu definieren. TAP-Messungen liefern im Ergebnis die 3D-Rekonstruktion des Analysevolumens. Je nach physikalischer Problemstellung kann dann - geeignete Software-Tools vorausgesetzt - eine Analyse erfolgen, die für die vorhandene Mikrostruktur optimiert ist. Der TAP-Detektor von der Firma CAMECA, Frankreich, wurde mit Software-Modulen ausgeliefert, die die grundlegenden Möglichkeiten der Visualisierung und Analyse ermöglichten. Als Basis für die TAP-Software diente die Software AVS, Version 5.3, von der Firma Advanced Visual Systems Inc., die auf einer Digital Personal Workstation 433 betrieben wurde. Der modulare Aufbau der AVS- und TAP-Basis-Software ermöglichte die Entwicklung einer eigenen, in der Programmiersprache C geschriebenen Software-Bibliothek, die in das bestehende System integriert wurde. Mit Hilfe dieser Software-Module konnten dann gezielte Analysen der untersuchten Nanostrukturen durchgeführt werden. Einen Überblick über die wichtigsten neu in dieser Arbeit entwickelten Analyse-Tools liefert Tab. 2.2. Bei den Analyse-Verfahren selbst handelt es sich um bekannte Verfahren, die zum Teil auch bei der konventionellen AP zum Einsatz kommen [MILL96].

\begin{tabular}{|l|l|}
\hline n-dim. FFT & $\begin{array}{l}\text { Mehrdimensionale Fast-Fourier-Transformation. } \\
\text { Ermöglicht die Suche nach Gitterebenen, 1D, 2D oder 3D } \\
\text { Autokorrelationen im Konzentrationsraum und weiteres mehr. }\end{array}$ \\
\hline $\begin{array}{l}\text { Konzentrationsprofil } \\
\text { - linear }\end{array}$ & $\begin{array}{l}\text { Interaktive Generierung von Konzentrationsprofilen. } \\
\text { Ermöglicht gezielte Konzentrationsanalysen in beliebigen Raum- } \\
\text { richtungen. }\end{array}$ \\
\hline $\begin{array}{l}\text { Konzentrationsprofil } \\
\text { - radial }\end{array}$ & $\begin{array}{l}\text { Generierung von radialen Konzentrationsprofilen. } \\
\text { Ermöglicht gezielte Konzentrationsanalysen von Grenzflächen } \\
\text { kugelförmiger kleinster Teilchen. }\end{array}$ \\
\hline Leiterdiagramm & $\begin{array}{l}\text { Interaktive Generierung von Leiterdiagrammen. } \\
\text { Vereinfacht insbesondere die Untersuchung von Grenzflächen. }\end{array}$ \\
\hline Autokorrelation & $\begin{array}{l}\text { 1D-Autokorrelation von Konzentrationsprofilen. } \\
\chi^{2} \text {-Anpassungstest }\end{array}$ \\
$\begin{array}{l}\chi^{2} \text {-Anpassungstest von Konzentrationsverteilungen. } \\
\text { Ermöglicht u. a. die Überprüfung der Homogenität eines Materi- } \\
\text { als. }\end{array}$ \\
\hline $\begin{array}{l}\text { Berechnung von Konzentrationen im 3D-Raum mit Moving- } \\
\text { Average-Funktionalität. } \\
\text { Dient als Grundlage für die Darstellung von Konzentrations- } \\
\text { querschnitten und Isokonzentrationsplots. }\end{array}$ \\
\hline
\end{tabular}

Tab. 2.2 Neu erstellte Software-Analyse-Tools für die Auswertung der 3D-Daten. 


\subsubsection{Spezielle Auswerteverfahren}

Die Auswertung der TAP-Messdaten erfolgt im nachhinein am Computer. Durch die große Menge an Informationen, die während einer Messung gewonnen werden können, steigt, im Vergleich zu konventionellen AP-Analysen, der Anteil der Zeit, der für die Auswertung aufgewendet werden muss. Die Auswerteverfahren sind dabei aber häufig an die konventionellen Verfahren angelehnt, wie sie in der Literatur beschrieben sind [MILL89, MILL96]. Mit Hilfe spezieller Software-Tools werden diese dann im 3DRaum umgesetzt.

\subsubsection{1 $\chi^{2}$-Anpassungstest}

Bei dem $\chi^{2}$-Anpassungstest handelt es sich um einen allgemein bekannten Test der mathematischen Statistik zur Prüfung der Hypothese, ob eine Zufallsgröße einem vorgegebenen Verteilungsgesetz genügt [BRON91]. Dieser Test kann auch auf APDaten angewendet werden, beispielsweise zur Überprüfung der Hypothese, ob eine Komponente in einem Material homogen verteilt ist oder nicht [MILL96]. $\chi^{2}$ Anpassungstests wurden auch im Rahmen dieser Arbeit mehrfach auf TAP-Messdaten angewendet. Die Implementierung dieses Verfahrens an der Göttinger TAP soll hier im folgenden kurz beschrieben werden.

Mit Hilfe eines speziell für diesen Test entwickelten Software-Moduls, s. a. Kap. 2.4.2, werden Stichproben mit einer festen Anzahl von Atomen innerhalb des Analysevolumens genommen. Die Stichproben beinhalten typischerweise 50 bis 200 nächste Nachbaratome um definierte Punkte im Volumen. Diese Punkte sind in diskreten Abständen über das gesamte Volumen verteilt, dergestalt, dass sich die Stichprobenvolumina nicht oder nur geringfügig überlappen. Eine Kontrolle darüber erfolgt über den mittleren Maximalabstand von den nächsten Nachbaratomen zum Stichprobenzentrum. Damit ist gewährleistet, dass die Stichproben unabhängig voneinander sind. Im Ergebnis erhält man schließlich die Häufigkeitsverteilung einer (ggf. auch mehrerer) ausgewählten Atomsorte in den Stichproben, die dann als Grundlage für einen $\chi^{2}$-Anpassungstest dient.

Bei einem Test bezüglich der Homogenität einer Legierung wird geprüft, ob die gemessene Häufigkeitsverteilung einer Binomialverteilung entspricht. Für eine homogene $\mathrm{A}_{\mathrm{c}}-\mathrm{B}_{(1-\mathrm{c})}$-Legierung wäre die Wahrscheinlichkeit $i$ A-Atome in einer Stichprobe mit $j$ Atomen zu finden:

$$
p_{i}^{(j)}=\left(\begin{array}{l}
j \\
i
\end{array}\right) \cdot c^{i} \cdot(1-c)^{j-i},(i=0,1, . ., j) .
$$

Bevor der $\chi^{2}$-Wert bestimmt werden kann muss zuvor noch eine geeignete Klasseneinteilung erfolgen [BRON91]. Für die Randklassen muss gelten $\left(N \cdot p_{i} \geq 1\right)$ und für alle weiteren Klassen $\left(N \cdot p_{i} \geq 5\right)$. Dabei ist $p_{i}$ die Wahrscheinlichkeit dass das Ergebnis einer Stichprobe in der i-ten Klasse liegt und $N$ die Gesamtzahl der erhobenen Stichproben. Der $\chi^{2}$-Wert berechnet sich dann aus

$$
\chi^{2}=\sum_{i=1}^{k} \frac{\left(M_{i}-N \cdot p_{i}\right)^{2}}{N \cdot p_{i}},
$$

wobei $k$ die Anzahl der Klassen und $M_{i}(i=1, . ., k)$ die zu der Klasseneinteilung gehörige gemessene Häufigkeitsverteilung ist. Dieser Wert wird verglichen mit einem kritischen Wert $\chi^{2}(a, r)$, wobei $a$ das Signifikanzniveau und $r=k-1$ die Zahl der 
Freiheitsgrade ist. Ist der gemessene $\chi^{2}$-Wert kleiner als der kritische $-\mathrm{d}$. $\mathrm{h}$. die Abweichungen von der Binomialverteilung sind verhältnismäßig klein -, so wird die Hypothese als bestätigt angesehen. Die Legierung ist homogen. Als Signifikanzniveau wird üblicherweise ein Wert von $a=0,05$ angesetzt.

\subsubsection{Konzentrationsprofile}

Konzentrationsprofile sind die klassische Form der Darstellung von Atomsonden-Daten. Während bei der konventionellen Atomsonde (AP) die Richtung des Profils und die Größe des Analysebereiches durch die Messrichtung bzw. die Größe der Atomsondenapertur bereits vor der Messung festgelegt werden, bietet die 3D-Atomsondentechnik (3DAP) die Möglichkeit im nachhinein in der 3D-Rekonstruktion des Probenvolumens einen beliebigen Analysebereich und eine Richtung zu definieren. Im Unterschied zur AP, bei der die Konzentrationsermittlung durch eine Einteilung der gemessenen AtomSequenzen in Blöcke mit konstanter Zahl von Atomen erfolgt, kann bei der 3DAP ein geeignetes Test-Analysevolumen definiert werden, dass Schritt für Schritt durch das (virtuelle) Probenvolumen gefahren wird und innerhalb dessen bei jedem Schritt eine lokale Konzentration bestimmt werden kann. So ist es möglich angepasst an die jeweilige Fragestellung ein optimiertes Analysevolumen zu verwenden. Bei der Ermittlung von Konzentrationen in einer 2D-Grenzfläche beispielsweise ist ein möglichst flaches, zylindrisches oder rechteckiges, Testvolumen optimal, dass Schritt für Schritt senkrecht durch die Grenzfläche gefahren wird.

Üblicherweise werden bei Konzentrationsprofilen zu den Messwerten auch die $2 \sigma-$ Grenzen mit angegeben, die zu einer mittleren Konzentration die - aus rein statitischen Gründen hervorgerufenen - möglichen Schwankungen des Messwertes anzeigen. Konzentrationen, die den $2 \sigma$-Bereich deutlich überschreiten, können so als signifikante Konzentrationsänderungen ausgemacht werden. Die Grenzen berechnen sich aus $2 \sigma=2 \times(\mathrm{c} \times(1-\mathrm{c}) /(\mathrm{N}-1))^{0,5}$, wobei c die mittlere Konzentration und $\mathrm{N}$ die Anzahl Atome im Analysevolumen ist. Da die Testvolumina eine konstante Größe haben, schwankt die Anzahl der Atome, die sich innerhalb derselben befinden. Dies ist hauptsächlich dadurch bedingt, dass nur etwa 50\% aller Atome detektiert werden, s. a. Kap. 2.4.4. Hinzu kommen noch lokale Variationen der Feldverdampfung auf den Spitzen. Als Folge ist deshalb auch der $2 \sigma$-Wert nicht konstant. Sofern die Konzentrationsänderungen groß sind ist es jedoch üblich dennoch einen mittleren Wert anzugeben. Sofern die Konzentrationsschwankungen weniger ausgeprägt sind, sollte jedoch zu jedem Konzentrationswert ein zugehöriger $2 \sigma$-Bereich angegeben werden. Bei den Konzentrationsprofilen in dieser Arbeit sind die 2 $\sigma$-Grenzen durchweg auf diese Art bestimmt worden.

\subsubsection{Experimentelle Parameter}

Die hier vorgestellten Untersuchungen wurden an drei Geräten durchgeführt: 1. an der Göttinger (konventionellen) Atomsonde [GEBE92], 2. an der Tomographischen Atomsonde in Rouen, Frankreich, [BLAV93] und 3. an der neuen Göttinger Tomographischen Atomsonde. Der Detektor der Göttinger TAP ist nahezu baugleich mit dem der TAP aus Rouen und ist von der Firma CAMECA, Frankreich. Die Bedingungen unter denen im FIM-Modus und im Atomsonden-Modus gearbeitet wurde, sowie die wichtigsten Parameter für die 3D-Rekonstruktion sind in Tab. 2.3 zusammengefasst. Für die Datenauswertung wurden einerseits die mit dem TAPDetektor gelieferte Software sowie selbst entwickelte Software verwendet, s. Kap. 2.4.2. 


\begin{tabular}{|l|l|c|c|}
\hline \multirow{2}{*}{ Modus } & \multicolumn{1}{|c|}{ Parameter } & Fe-Ag & Ni-P \\
\hline \multirow{2}{*}{ FIM } & Bildgase & $\mathrm{Ne}+\mathrm{H}_{2}\left(<10^{-7} \mathrm{hPa}\right)$ & $\mathrm{Ne}$ \\
\cline { 2 - 4 } & $\mathrm{T}_{\mathrm{KK}}$ & $20 \mathrm{~K}$ & $35-50 \mathrm{~K}$ \\
\hline \multirow{3}{*}{ TP / } & Pulsverhältnis $\mathrm{U}_{\mathrm{P}} / \mathrm{U}_{\mathrm{DC}}$ & $0,15-0,25$ & 0,18 \\
\cline { 2 - 4 } & Pulsfrequenz & $1-2 \mathrm{kHz}$ & $2 \mathrm{kHz}$ \\
\cline { 2 - 4 } & TAP-Detektionsrate [Ionen/Puls] & $0,01-0,1$ & 0,01 \\
\cline { 2 - 4 } & Feldverdampfungsparamter $\mathrm{k} \cdot \mathrm{F}_{\mathrm{f}}$ & $18 \mathrm{~V} / \AA$ & $21 \mathrm{~V} / \AA$ \\
\cline { 2 - 4 } & Bildkompressionsfaktor b & 1,6 & 1,6 \\
\cline { 2 - 4 } & Detektionseffizienz & 0,5 & 0,5 \\
\cline { 2 - 4 } & $\mathrm{T}_{\mathrm{KK}}, \mathrm{AP}$ & $20 \mathrm{~K}$ & - \\
\cline { 2 - 4 } & $\mathrm{T}_{\mathrm{KK}}, \mathrm{TAP}$ & $50 \mathrm{~K}$ & $25-50 \mathrm{~K}$ \\
\hline
\end{tabular}

Tab. 2.3 Parameter für AP- bzw. TAP-Untersuchungen

Die in der Tab. 2.3 angegebenen Werte $\mathrm{T}_{\mathrm{KK}}$ sind die Kryostattemperaturen. Bedingt durch die Verluste bei der thermischen Ankopplung an die Probenhalter liegen die Temperaturen der Proben-Spitzen in etwa 10 bis $15 \mathrm{~K}$ höher.

Bei den AP-Analysen der Fe-Ag-Legierungen wurden die tiefsten möglichen Temperaturen und teilweise auch relativ große Pulsverhältnisse eingestellt. Dadurch wurde versucht einen eventuellen Effekt der bevorzugten Feldverdampfung [MILL96] auszuschließen, der im allgemeinen bei anderen bekannten Legierungssystemen dann nicht mehr auftritt [MILL96]. Diesen Extrembedingungen hielten die Fe-Ag-Schichten auf den Wolfram-Spitzen jedoch nicht lange stand. Messungen unter diesen erschwerten Bedingungen konnten zwar erfolgreich durchgeführt werden, aber nur in geringer Anzahl. Die TAP-Analysen wurden aufgrund dieser Erfahrung bei höherer Temperatur mit Pulsverhältnissen von 0,15 durchgeführt. Die dadurch bedingten eventuellen Abweichungen von realen und gemessenen Legierungszusammensetzungen wird mit nicht mehr als einem Faktor 2 abgeschätzt.

Die TAP-Analysen der Ni-P-Legierungen im Zustand nach der Herstellung zeigten einen klaren Zusammenhang zwischen Probentemperatur und gemessenem mittlerem PGehalt. In Abb. 2.3 ist dieser am Beispiel einer Ni-5,9At.\%-P-Legierung dargestellt. Phosphor wird offensichtlich bevorzugt feldverdampft. Bei Kryostat-Temperaturen von 15 bis $20 \mathrm{~K}$ wird jedoch die nominelle Konzentration getroffen. Wie bereits bei den FeAg-Legierungen der Fall, war bei tiefen Temperaturen zu beobachten, dass die Proben mechanisch sehr instabil wurden und häufig während der Messung abrissen. Interessanterweise nahm die Stabilität merklich zu, sobald die Proben thermisch behandelt wurden. Bei den angelassenen Proben wurde i. d. R. auch bereits bei KryostatTemperaturen von $25 \mathrm{~K}$ die nominelle Konzentration als mittlere Konzentration bestimmt. 


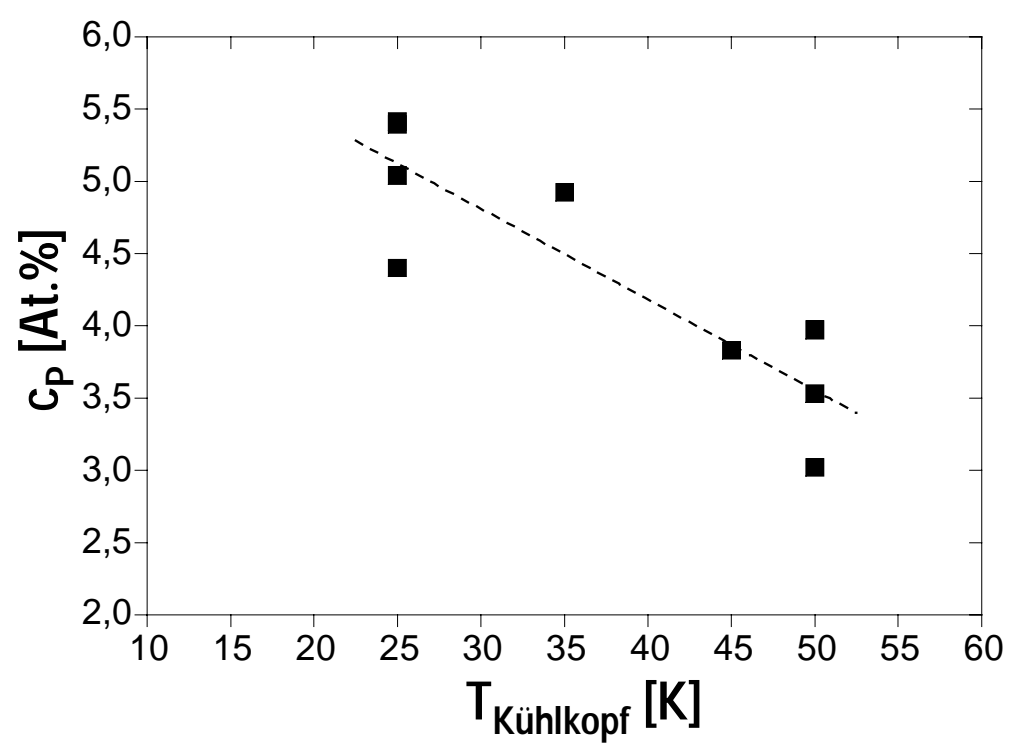

Abb. 2.3 Mittlerer gemessener P-Gehalt einer Ni-5,9At.\%-P-Legierung bei unterschiedlichen KryostatTemperaturen.

Die Detektions-Effizienz des TAP-Detektors wurde für die 3D-Rekonstruktion mit 0,5 veranschlagt. Der wesentliche Anteil am Verlust von Ionen ist bedingt durch den Anteil an inaktiver Fläche der MCPs. Ihre Detektionseffizienz beträgt ca. 0,55-0,60. Sie ist, weil rein geometrisch bedingt, von der auftreffenden Ionen-Spezies unabhängig. Hinzu kommt noch eine Detektor spezifische Effizienz von ca. 0,9, die auf Elektronen-SpotÜberlappungsprobleme sowie auf Ereignisse mit unbrauchbaren Elektronen-SpotGrößen zurückzuführen ist. Letztgenannter Effekt ist zwar Ionen spezifisch - er tritt im wesentlichen beim Vorhandensein von unterschiedlichen Ionisationsgraden und damit verbundenen unterschiedlichen kinetischen Energien auf - , ist aber durch geeignete Justage der Verstärkung der MCPs relativ gering zu halten. Ist ein Elektronenspot dennoch beispielsweise zu klein, so kann zwar die spezifische Masse nachwievor ermittelt werden, die Position ist jedoch auf der Länge eines Elektrodenfeldes (entspricht 1 bis $2 \mathrm{~nm}$ in der 3D-Rekonstruktion) unbestimmt. Für die hier vorgestellten Untersuchungen wurden daher in den meisten Fällen die nicht exakt positionierbaren Atome herausgefiltert und in der 3D-Rekonstruktion nicht berücksichtigt. Ihr Anteil lag bei ca. $12 \%$. Für die Bestimmung von mittleren Konzentrationen jedoch wurden jeweils alle Atome berücksichtigt. Beim Herausfiltern der nicht exakt positionierbaren Atome ergaben sich im Vergleich dazu bei den Ni-P-Legierungen keine relevanten Änderungen der mittleren Konzentration (2-3\% vom Messwert). Bei den Fe-Ag-Legierungen war der Ionen spezifische Effekt (s.o.) jedoch relativ stark. Ergänzende Messungen allein zur systematischen Optimierung der Detektorverstärkung wurden aufgrund des hohen präparativen Aufwandes nicht durchgeführt. Nicht exakt positionierbare Ag-Atome traten deshalb im Verhältnis häufiger auf als dies bei Fe-Atomen der Fall war. Der Einfluss auf den mittleren Ag-Gehalt lag bei ca. 20 bis 25\% vom jeweiligen Messwert.

Der Feldverdampfungsparameter $\mathrm{kF}_{\mathrm{f}}$ für $\mathrm{Fe}$-Ag-Legierungen wurde anhand von Literaturwerten [MILL96] für reines Eisen abgeschätzt. Als Wert für die Ni-PLegierungen wurde der reinen Nickels verwendet. Mit Hilfe von TAP-Analysen an NiSpitzen wurde durch Eichung der in der 3D-Rekonstruktion sichtbaren (111)-Gitterebenenabstände der Wert für $\mathrm{kF}_{\mathrm{f}}$ mit 20-21 V/Å bestimmt.

Die Massenspektren bei den AP- und TAP-Analysen konnten ohne Schwierigkeiten ausgewertet werden. Insbesondere gab es keine Peak-Überlappungen. Eisen- und 
Nickel-Ionen wurden größtenteils als $\mathrm{Fe}^{2+}$ bzw. $\mathrm{Ni}^{2+}$ detektiert. Silber erschien ausschließlich bei den spezifischen Massen 109 und 111 als Dihydrid-Ion $\left(\mathrm{AgH}_{2}\right)^{+}$im Spektrum. Phosphor (Masse 31) trat zweifach und in geringerem Umfang dreifach ionisiert als $\mathrm{P}^{2+}$ und $\mathrm{P}^{3+}$ auf. Dieses Verhalten ist konsistent mit Angaben in der Literatur [MILL96-Anhang H]. Einfach ionisierter Phosphor ließe sich zwar nicht von dem zweifach ionisierten Nickel Isotop ${ }^{62} \mathrm{Ni}^{2+}$ unterscheiden, da aber unter geeigneten Versuchsbedingungen, s.o., die nominellen P-Gehalte mit den gemessenen mittleren PKonzentrationen übereinstimmten kann die Bildung von $\mathrm{P}^{+}$-Ionen ausgeschlossen werden.

\subsection{Weitere Untersuchungsmethoden}

Mit der Elektronenstrahlmikrosonde (EMS) kann die chemische Zusammensetzung von Legierungen präzise bestimmt werden. Das lokale Auflösungsvermögen liegt dabei bei wenigen Mikrometern. Die Ni-P-Legierungen wurden zur Bestimmung der absoluten mittleren P-Konzentration mit einer EMS der Firma CAMECA, Modell SX-50, untersucht. Die nominellen Konzentrationsangaben bei Ni-P-Legierungen in dieser Arbeit basieren auf diesen EMS-Messungen.

Zur Charakterisierung vor TAP-Analysen sowie vor und nach Beschichtungen von TAP-Spitzen wurden speziell präparierte Folien und AP/TAP-Spitzen in einem Transmissions-Elektronenmikroskop (TEM), EM400T, der Firma Philips untersucht. Das Gerät war mit einer $\mathrm{LaB}_{6}$-Kathode ausgestattet und wurde bei $120 \mathrm{kV}$ betrieben. Die Untersuchungen erfolgten in konventioneller Hellfeldabbildung (CTEM) [REIM67].

Für die thermische Analyse der Ni-P-Legierungen wurde die Differential-ScanningCalorimetry (DSC) angewendet. Es wurden Thermogramme mit Hilfe einer DSC-7 der Firma Perkin-Elmer aufgenommen. Die Proben wurden dabei in Aluminium-Pfännchen mit perforiertem Deckel verpackt und von einem kontinuierlichen Argon-Gas-Strom umspült. Die Heizexperimente wurden isochron mit einer konstanten Heizrate von $5 \mathrm{~K} / \mathrm{min}$ geführt. Bei diesem Verfahren wird die Differenz der Heizleistung zwischen der Probe und einer inerten Referenz als Funktion der Temperatur gemessen. Als Referenz diente ein leeres Aluminium-Pfännchen. Die Proben wurden bei der selben Heizrate in zwei aufeinander folgenden Durchläufen von Raumtemperatur auf jeweils $600{ }^{\circ} \mathrm{C}$ geheizt. Die Messkurve des zweiten Durchlaufes diente dann als Referenzkurve und wurde von der ersten subtrahiert. Auf diese Art entstanden Thermogramme der irreversiblen mikrostrukturellen Umwandlungen in den Ni-P-Legierungen. Exotherme Reaktionen wurden immer als nach unten zeigende Peaks dargestellt. Für die Auswertung der Thermogramme konnte auf die Auswertesoftware von Perkin-Elmer zurückgegriffen werden.

Die Röntgendiffraktometrie (XRD) ist eine Standardmethode zur Untersuchung von Festkörpern [CULL67, WARR69]. Die Ni-P-Legierungen wurden mit Hilfe eines kommerziellen Vierkreis-Diffraktometers der Firma Philips, Modell X'Pert, untersucht. Die verwendete Röntgenstrahlung hatte eine Wellenlänge von $\lambda=0,17903 \mathrm{~nm}\left(\mathrm{Co}-\mathrm{K}_{\alpha}\right)$. Es wurden Diffraktogramme in konventioneller $\Theta-2 \Theta-$ Strahlgeometrie $^{5}$ erstellt, mit $^{-}$ deren Hilfe das Auftreten neuer Phasen nach Wärmebehandlungen untersucht wurde. Desweiteren wurden aus den Peakverbreiterungen der Ni(P)-(111)-Reflexe die mittleren

\footnotetext{
${ }^{5} \Theta$ ist der Bragg-Winkel
} 
Korngrößen D der nanokristallinen Legierungen mit Hilfe der Scherrer-Formel [CULL67, WARR69] bestimmt:

$$
D=\frac{0,9 \cdot \lambda}{B \cdot \cos \Theta}
$$

Der Faktor B ist die Halbwertsbreite der Peaks in der $2 \Theta$-Auftragung in Radiant. Die apparative Peakverbreiterung wurde mit Hilfe einer reinen Nickel-Folie bestimmt. Sie betrug lediglich 0,1 Grad. Eine Entfaltung von Messpeaks und apparativem Peak wurde deshalb nicht vorgenommen. Der Fehler bei der Bestimmung der mittleren Korngrößen beträgt ohnehin ca. 10-30\% [KRIL98]. Insbesondere ist der Einfluss von eventuellen Stapelfehlern und Zwillingskorngrenzen hier nicht bestimmbar.

Das Philips Diffraktometer ermöglichte zusätzlich auch die Untersuchung von kristallographischen Texturen [CAHN96] der Ni-P-Schichten. So konnten die

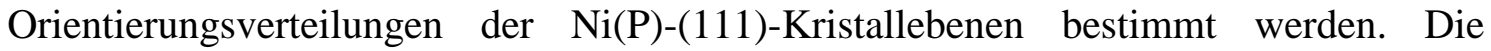
Ergebnisse der Texturuntersuchungen wurden mit Hilfe der Diffraktometer-eigenen Software in Form von Höhenliniendiagrammen (Polfiguren) aufbereitet. 


\section{Untersuchungen an Fe-Ag-Legierungen}

Mit Hilfe der gepulsten Laserdeposition (PLD) wurden Fe-Ag-Legierungen auf vorbereitete Wolfram-Spitzen deponiert, wie in Kap. 2.1 beschrieben. Parallel zu den Spitzen wurden zusätzlich unter denselben Bedingungen auch planare SiliziumSubstrate beschichtet. Der mittlere Ag-Gehalt in den planaren Schichten konnte mit EDX bestimmt werden und diente als Referenz. Es wurden in mehreren Versuchsreihen zunächst Schichten mit Ag-Gehalten zwischen 7 und 9 At.\% und im Anschluss daran Schichten mit Konzentrationen zwischen 20 und 30 At.\% Ag (jeweils gemessen auf dem planaren Substrat) hergestellt. Die Schichtdicken auf den planaren Substraten betrugen zwischen 50 und $150 \mathrm{~nm}$.

Auf den planaren Substraten wurden zusätzlich zu den Schichten auch Mikrometer große Fe- und Ag-Tröpfen gefunden. Die Bildung von Tröpfen bei der PLD ist ein bekannter, eher unerwünschter Effekt [BENN95, FÄHL97]. Bei der Deposition auf Spitzen spielten sie jedoch keine Rolle. Töpfchen, die unweigerlich die filigranen Spitzen bei einer Kollision zerstört hätten, wurden nicht beobachtet.

\subsection{Schichten auf Wolfram-Spitzen}

Nach der Deposition der Fe-Ag-Schichten wurden die beschichteten Spitzen im TEM untersucht. Dabei zeigte sich, dass die Schichtdicken im Vergleich zu den planaren Referenz-Substraten erheblich geringer ausfielen. Am Apex betrugen sie etwa die Hälfte bis ein Drittel der planaren Schichtdicken. Außerdem wiesen die Schichten in den meisten Fällen eine charakteristische Kappenform auf, die einem Kegel glich. Zwei Beispiele derarter Spitzen sind in Abb. 3.1 dargestellt. Es sind jeweils die W-SubstratSpitzen vor und nach der Beschichtung dargestellt. Die Spitze (A) wurde mit einem nominell 7,2 At.\%igen, die Spitze (B) mit einem 21,3 At.\%igen Film beschichtet. Bei Spitzen aus den ersten Versuchsreihen mit geringeren nominellen Ag-Gehalten war dieser Effekt ausgeprägter. Diese zeigten auch am Spitzenschaft ähnliche Erscheinungen. Ihre Form erinnerte ein wenig an die von Tannenzapfen, s. Abb. 3.1 (A).

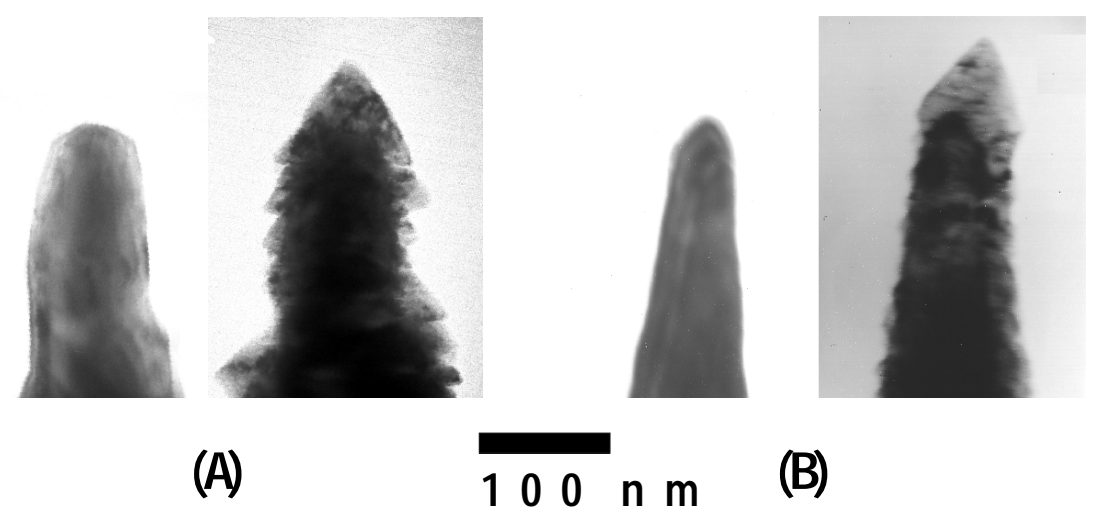

Abb. 3.1 TEM-Aufnahmen von W-Spitzen vor und nach der Deposition von Fe-Ag-Schichten mittels PLD. Der Ag-Gehalt der planaren Referenz-Proben betrug (A) 7,2 At.\% und (B) 21,3 At.\%.

\subsection{Struktur der Schichten}

Bei Schichten mit höheren Ag-Gehalten wurden im TEM kleine Kristallite von der Größenordnung $15 \mathrm{~nm}$ auf den Spitzenapexes beobachtet. In Abb. 3.2 ist eine Spitze 
nach der Beschichtung in Dunkelfeldabbildung dargestellt. Der Ag-Gehalt der planaren Referenzschicht betrug hierbei 21,3 At.\%. Deutlich zu erkennen ist die Substratspitze und die darauf befindliche Schicht, sowie die kleinen Körner in der Schicht. Bei geringerem Ag-Anteil waren diese nicht zu beobachten. Vielmehr zeigten sich gröbere Kornstrukturen, die nur bedingt abbildbar waren, da die Spitzen im TEM nicht gekippt werden konnten.

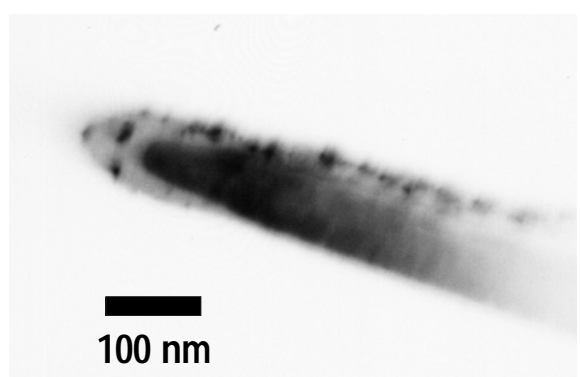

Abb. 3.2 Fe-Ag-Schicht auf einer W-Spitze (TEM-
Aufnahme in Dunkelfeldabbildung). Kleine, ca. $15 \mathrm{~nm}$
messende Kristallite sind zu erkennen. Der Ag-Gehalt der
planaren Referenzschicht beträgt 21,3 At. $\%$.

Ein wesentlich klareres Bild lieferten dann die anschließenden FIM-Untersuchungen. Fe-Ag-Schichten mit Ag-Konzentrationen von 7 bis 9 At.\% (planare Referenzschicht) zeigten überaschenderweise eine auf der FIM-Skala einkristalline Struktur. Die mit FIM abbildbaren Bereiche der Spitzenapexes wiesen eine durchgehende Polstruktur auf, die der von kubisch raumzentrierten (bcc) Einkristallen entspricht. Die Pole waren deutlich $\mathrm{zu}$ erkennen, so dass eine vollständige kristallographische Indizierung durchgeführt werden konnte. In Abb. 3.3 (A) ist das FIM-Bild einer solchen Spitze mit der dazugehörigen Indizierung dargestellt. Der (011)-Pol in der Mitte des Bildes ist zum Teil von der Atomsondenapertur (dunkler kreisförmiger Fleck) abgedeckt.

Durch kontinuierliches Feldverdampfen konnten dann die Fe-Ag-Schichten unter Beobachtung im FIM sukzessive abgetragen werden bis die Grenzfläche zum WolframSubstrat sichtbar wurde. In Abb. 3.3 (B) ist ein solcher Zustand abgebildet ${ }^{6}$. In einem kleinen Bereich durchstößt das W-Substrat bereits die Oberfläche. Für Wolfram ist charakteristisch, dass es mit Neon - dem für die Fe-Ag-Schicht verwendeten Bildgas anders abbildet. Es zeigte sich deshalb in der FIM-Abbildung in diesem Bereich zunächst ein nebelartiges, milchiges Bild. Durch zusätzliches Einlassen von Helium war es jedoch möglich, auch die Polstruktur des Wolfram in diesem Bereich abzubilden.

Durch fortgesetztes Feldverdampfen wurden größere Bereiche der W-Spitze sichtbar. In vielen Fällen rissen auch die Schichten als Ganzes, unter dem Einfluss der durch das elektrische Feld hervorgerufenen mechanischen Spannungen, von den Spitzen ab, so dass am Ende die W-Spitzen zum Vorschein kamen. Diese wurden dann mit Helium als Bildgas abgebildet. Die Polstrukturen der nun sichtbaren Bereiche der W-Spitzen konnten anschließend kristallographisch eindeutig indiziert werden. In Abb. 3.3 (C) ist dieser Zustand an einem Beispiel dargestellt.

Die Auswertung einer gesamten Sequenz, wie sie beispielsweise in Abb. 3.3 dargestellt ist, gab unmittelbar Aufschluss über die Orientierungsbeziehung zwischen Schicht und Substrat. Die untersuchten Fe-Ag-Schichten mit Ag-Gehalten von 7 bis 9 At.\% (planare Referenzschicht) zeigten im Ergebnis alle eine Übereinstimmung der

\footnotetext{
${ }^{6}$ Im Vergleich zu Bild (A) ist die Spitze hier ein wenig nach links verkippt worden.
} 
kristallographischen Orientierung mit der des W-Substrats - eine Abweichung von wenigen Grad kann dabei jedoch nicht ausgeschlossen werden. Das Wachstum dieser Fe-Ag-Schichten auf den W-Substratspitzen war also durch Epitaxie gekennzeichnet.
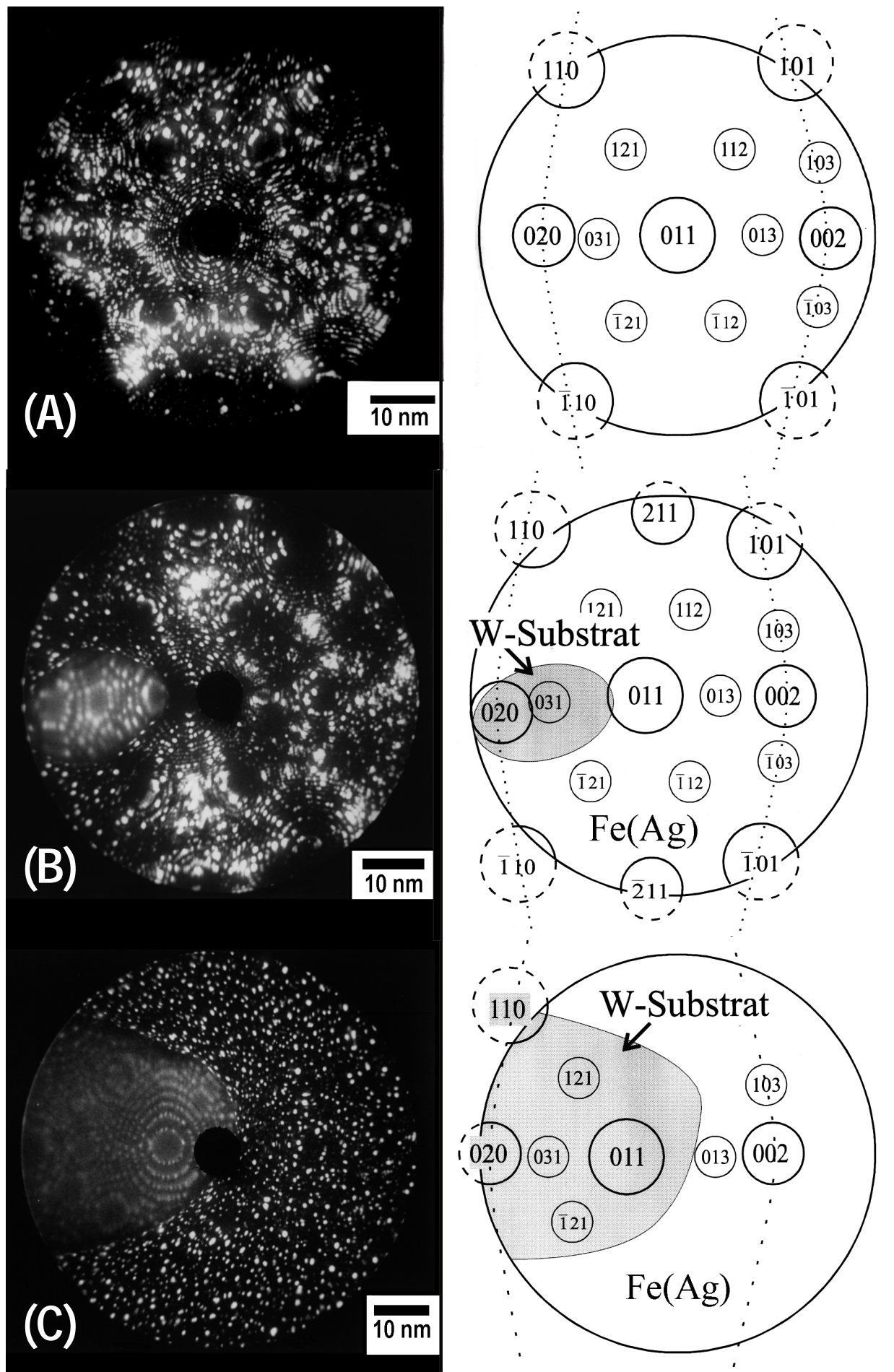

Abb. 3.3 FIM-Sequenz einer Fe-Ag-Schicht auf einer W-Substratspitze. Die nominelle AgKonzentration der Schicht, gemessen an der planaren Referenzschicht, betrug 7,2 At.\%. Jeweils links sind die FIM-Bilder unterschiedlicher Entwicklungsstadien dargestellt: (A) Fe-Ag-Schicht mit vollständig entwickelter Polstruktur; (B) das W-Substrat tritt hervor an die Oberfläche; (C) weiterentwickeltes Stadium. Jeweils rechts sind die dazugehörigen kristallographischen Inidzierungen skizziert. Die Sequenz demonstriert unmittelbar die Epitaxie der Fe-Ag-Schicht. 
Bei den FIM-Untersuchungen wurden keine zusätzlichen Kontraste in den FIM-Bildern beobachtet, die das Vorhandensein von Ag-Ausscheidungen oder Bereichen unterschiedlicher chemischer Zuammensetzung hätten vermuten lassen können. Dies gilt für alle durchgeführten Untersuchungen. Auch bei Schichten mit höheren AgGehalten. Letztgenannte zeigten jedoch im FIM ein deutlich anderes Bild. Einkristalline oder Grobkristalline Strukturen konnten hier nicht beobachtet werden. Es waren jedoch zum Teil vereinzelte Pole in kleinen (Größenordnung $10 \mathrm{~nm}$ ) Bereichen sichtbar. In Abb. 3.4 ist das Beispiel einer FIM-Abbildung einer Fe-Ag-Schicht mit einer nominellen (auf dem planaren Referenzsubstrat gemessenen) Konzentration von 21,3 At.\% Ag dargestellt. Die Pfeile im unteren Bildbereich deuten die Position eines Poles an. Der Pol bildet vergleichsweise dunkel ab, so dass andere Bereiche der noch nicht vollständig entwickelten Spitze hier stark überstrahlen. Kontraste, die auch im weiteren Verlauf der Spitzenentwicklung auf Ausscheidungen hingedeutet hätten wurden nicht beobachtet.

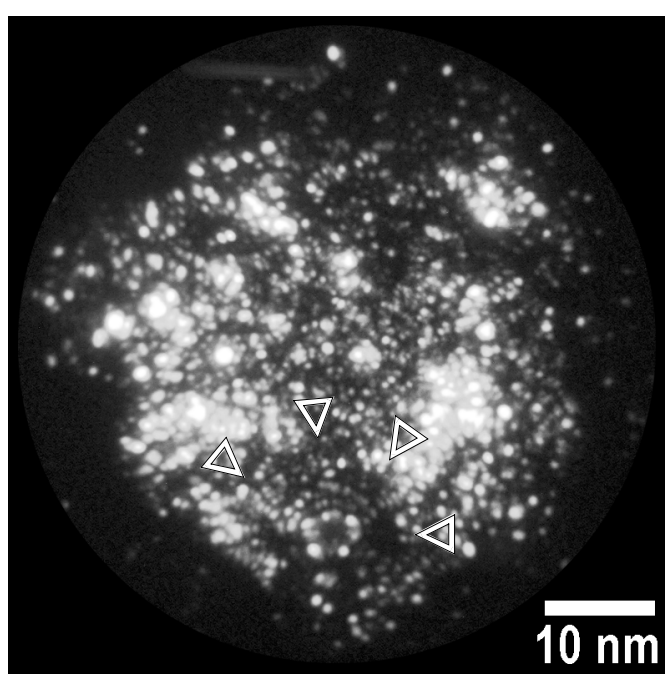

Abb. 3.4 FIM-Bild einer Fe-Ag-Schicht mit nominell 21,3 At.\% Ag (gemessen an planarer Referenzschicht). Mit Pfeilen gekennzeichnet ist ein kristallographischer Pol, der in einem kleinen Bereich sichtbar ist.

\subsection{Chemische Zusammensetzung der Schichten}

Die planaren Fe-Ag-Referenzschichten aus den ersten Versuchsreihen wiesen einen mit EDX bestimmten Ag-Gehalt von 7 bis 9 At.\% auf. Mit Hilfe konventioneller Atomsonde (AP) wurden die auf W-Spitzen aufgebrachten Schichten untersucht, um auch hier die Zusammensetzung zu bestimmen. Einerseits konnten so die mittleren AgGehalte überprüft und andererseits die Verteilung des Silbers in den untersuchten Probenvolumina auf einer nm-Skala analysiert werden. Da die FIM-Untersuchungen keine Hinweise auf besondere auffällige $\mathrm{zu}$ analyierende Probenbereiche ergaben, wurden „Random-Area-Analysen“ durchgeführt, d. h. Analysen an verschiedenen willkürlich ausgewählten Positionen. Die mechanische Stabilität der Spitzen erwies sich bei den Messungen als merkliches Problem. Die Mehrzahl der Proben wurden durch Abriss der Schichten während der Messungen zerstört. Bei drei Schichten gelang es aber längere Analysen über eine Tiefe von jeweils ca. $10 \mathrm{~nm}$ durchzuführen. Das Ergebnis einer solchen Messung ist in Abb. 3.5 als Leiterdiagramm dargestellt. Bei dieser Form der Darstellung wird die kumulierte Anzahl der detektierten Ag-Ionen, ihrer gemessenen Reihenfolge entsprechend, gegen die kumulierte Anzahl aller detektierten Ionen, d. h. Eisen und Silber, aufgetragen. Die Steigung der Kurve entspricht der lokalen Ag-Konzentration. 


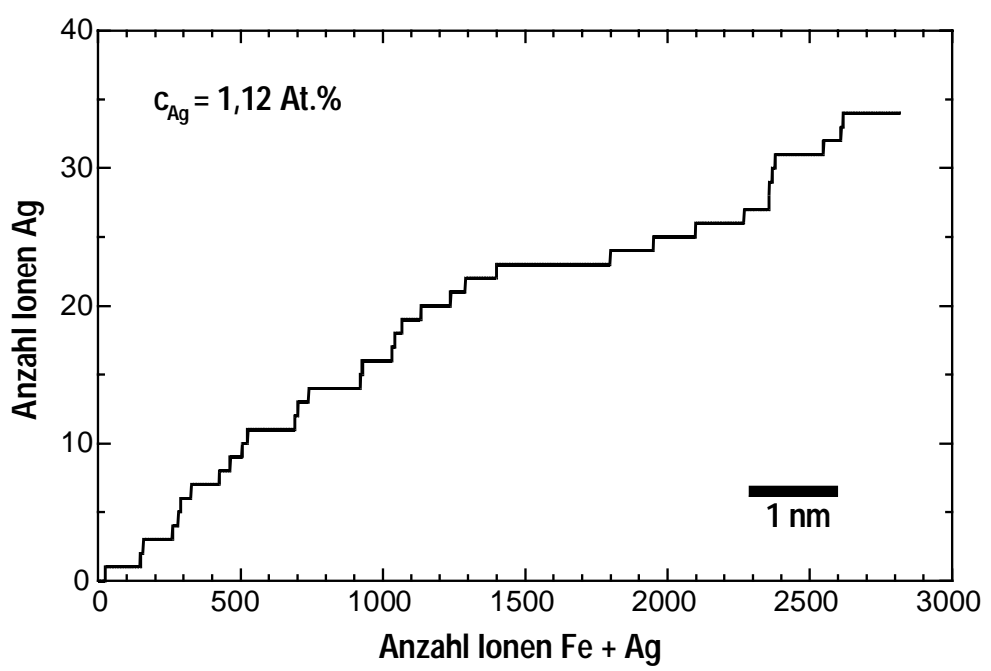

Abb. 3.5 AP-Analyse einer Fe-Ag-Schicht, dargestellt als Leiterdiagramm. Der mittlere Ag-Gehalt wurde hier mit 1,12 At.\% bestimmt. Die Konzentrationsschwankungen liegen innerhalb der 2- $\sigma$-Grenzen. Die Ag-Konzentration der planaren Referenzschicht betrug in diesem Fall 7,2 At.\%.

Die gemessene mittlere Ag-Konzentration dieser Analyse, sowie auch die der anderen Analysen betrug 1,1 At.\%. Diesem Wert stehen die nominellen mittleren Ag-Gehalte von 7,2 und 8,0 At.\% gegenüber, die mittels EDX an den planaren Referenzschichten bestimmt wurden. Die lokalen Ag-Konzentrationen in den Schichten blieben über den Verlauf der jeweiligen Messung konstant, d. h. sie lagen im Rahmen der natürlichen statistischen Schwankungen innerhalb des 2- $\sigma$-Bereiches. Die Schichten aus der ersten Versuchsreihe sind damit als homogen zu bezeichnen bei mittleren Konzentrationen von 1,1 At. $\%$ Ag.

Für die Untersuchung der Fe-Ag-Schichten der zweiten Versuchsreihe mit nominellen Ag-Konzentrationen (planare Referenzschicht) von 20 bis 30 At.\% stand die Tomographische Atomsonde (TAP) zur Verfügung. Auch bei diesen Schichten war die mechanische Stabilität der Schichten gering. Es konnten aber zwei längere Messungen erfolgreich durchgeführt werden mit 100000 bzw. 70000 detektierten Ionen sowie eine kurze mit 8000 Ionen. Eine der beiden längeren Messungen durchläuft die Grenzfläche zum W-Substrat. Die 3D-Rekonstruktion des analysierten Probenvolumens von $16 \times 16 \times 7 \mathrm{~nm}^{3}$ ist in Abb. 3.6 dargestellt. Gelbe Punkte entsprechen Fe-Atomen, rote AgAtomen und grüne $\mathrm{W}$-Atomen.

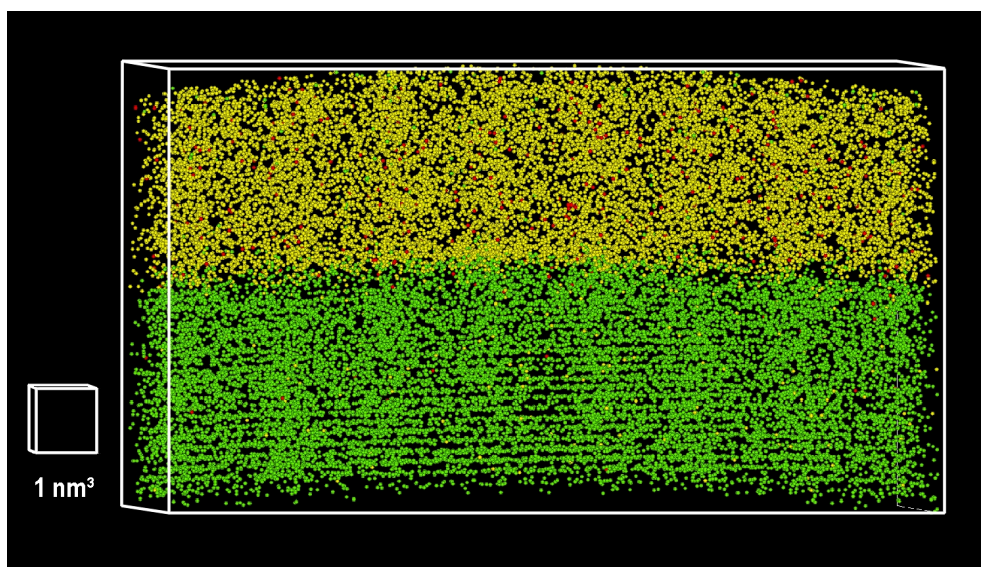

Abb. 3.6 Fe-Ag-Schicht auf einem W-Substrat in der 3DRekonstruktion nach einer TAPAnalyse. Das Analysevolumen beträgt $16 \times 16 \times 7 \mathrm{~nm}^{3}$. Fe-Atome sind gelb, Ag-Atome rot und WAtome grün dargestellt. Der mittlere Ag-Gehalt der Schicht beträgt 3,0 At.\%. 
Im oberen Teil ist die Fe-Ag-Schicht zu sehen, im unteren das W-Substrat. Das Volumen ist so orientiert, das die Wolfram-(110)-Ebenen sichtbar sind. ${ }^{7}$ Der mittlere Ag-Gehalt in der Fe-Ag-Schicht betrug hier 3,0 At.\%. Im Vergleich dazu wurde in der planaren Referenzschicht ein Wert von 28,5 At\% Ag mittels EDX bestimmt. Die zweite erfolgreiche längere Messung ergab eine mittlerer Ag-Konzentration von 2,8 At.\%, wobei dieselbe Referenzkonzentration wie bei der ersten vorlag. Wie bereits bei den Untersuchungen mit konventioneller AP musste auch in diesem Fall eine große Diskrepanz zwischen gemessenen Ag-Konzentrationen in der Schicht und nominellen Ag-Konzentrationen in den planaren Referenzschichten festgestellt werden. Die dritte nur kurze Messung war zwar für eine weitere statistische Analyse nicht umfangreich genug, sie bestätigte aber diesen Trend: Der gemessenen Ag-Konzentration von 2,7 At.\% stand eine nominelle von 21,3 At.\% gegenüber.
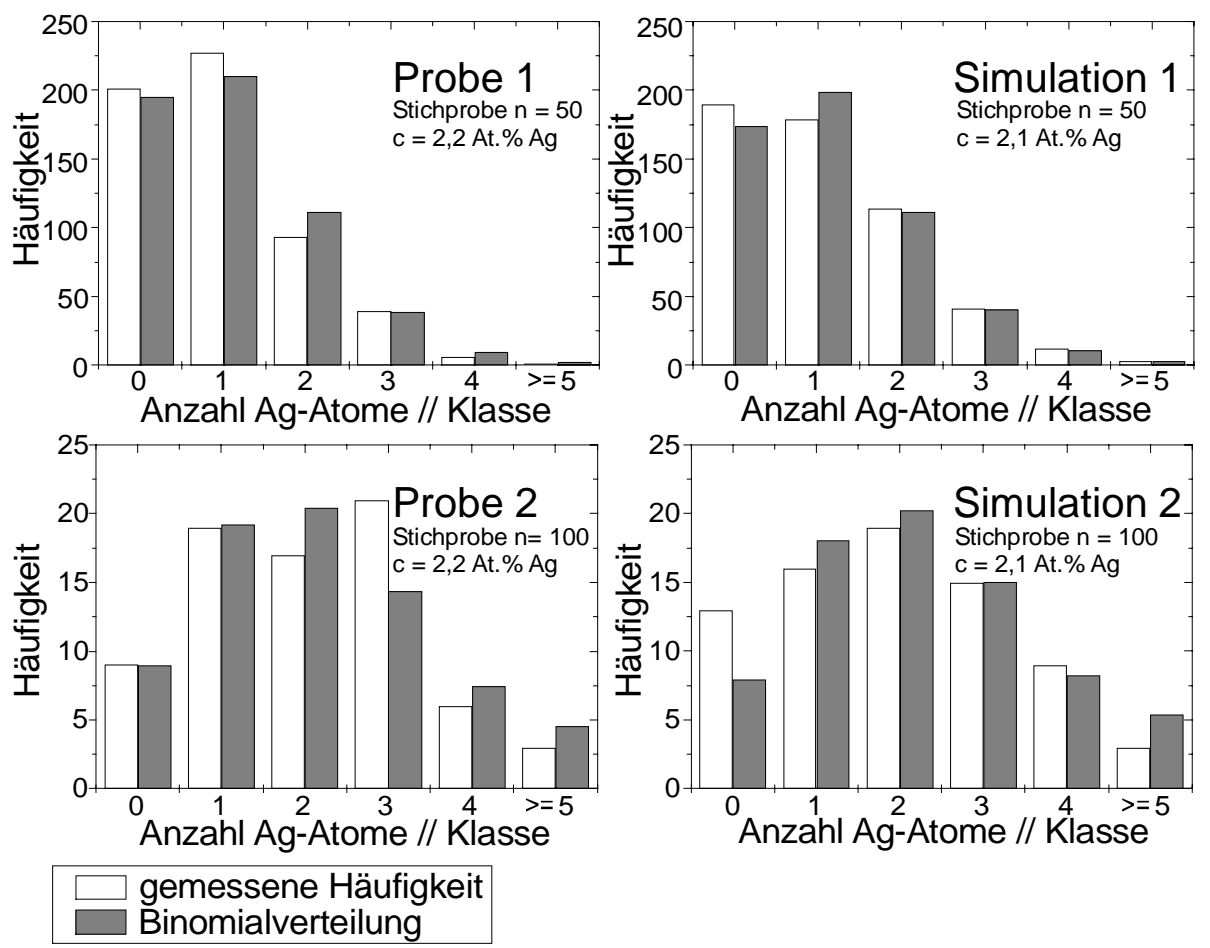

gemessene Häufigkeit

Binomialverteilung

Abb. 3.7 Häufigkeitsverteilungen von Ag-Atomen in Stichproben. Weiße Balken entsprechen gemessenen Verteilungen, graue Balken entsprechen den korrespondierenden Binomialverteilungen. Den Ergebnissen von Probe 1 und Probe 2 sind jeweils Ergebnisse von simulierten homogenen Legierungen gegenübergestellt. Die Häufigkeitsverteilungen dienen als Basis für $\chi^{2}$-Anpassungstests, s. Text.

Die Verteilung des Silbers innerhalb der Analysevolumina war augenscheinlich homogen. Auch Konzentrationsprofile durch die Analysevolumina zeigten keine statistisch signifikanten Ag-Anreicherungen. Dies führte zu der Schlussfolgerung, dass die Fe-Ag-Schichten insgesamt homogen seien. Um diese Homogenitäts-Hypothese mit den Mitteln der Statistik endgültig abzusichern wurden $\chi^{2}$-Anpassungstests, s. Kap. 2.4.3, durchgeführt. Es wurden an zwei verschiedenen Proben (Probe 1 und 2) Stichproben mit 50 und mit 100 Atomen genommen. Dabei wurden ausschließlich die Atome berücksichtigt, die auf dem TAP-Detektor exakt positionierbar waren (ca. 87\% aller Atome), um artifizielle Einflüsse auf die Häufigkeitsverteilungen auszuschließen.

\footnotetext{
${ }^{7}$ Der Abstand ist nicht ganz maßstabsgetreu, da die Abbildungsparameter an Eisen angepasst wurden und nicht an Wolfram.
} 
Wie in Kap. 2.4.4 beschrieben, wurden dadurch präferentiell Ag-Atome herausgefiltert. Die mittlere Konzentration der Analysevolumina verringerte sich daduch von 2,8 und 3,0 auf jeweils 2,2 At.\% Ag. In Abb. 3.7 sind die resultierenden Häufigkeitsverteilungen und die korrespondierenden hypothetischen Binomialverteilungen homogener Legierungen gleicher mittlerer Konzentration (2,2 At.\% Ag) dargestellt. Zum Vergleich wurden desweiteren zwei homogene Fe-2,1At.\%Ag-Legierungen simuliert und unter denselben Bedingungen wie die Proben 1 und 2 analysiert. Die dazugehörigen Ergebnisse sind auch in Abb. 3.7 dargestellt.

Es zeigte sich, dass die gemessenen Häufigkeitsverteilungen und die Binomialverteilungen nahezu identisch waren. Die ermittelten $\chi^{2}$-Werte betrugen lediglich 3,6 bzw. 4,7. Für die simulierten homogenen Legierungen wurden Werte von 4,5 und 6,6 ermittelt. Im Vergleich dazu lag der kritische Wert $\chi^{2}(a=0,05)$ bei 12,6 weit entfernt. Damit wurde die Homogenitäts-Hypothese unzweifelhaft bestätigt. Die Schichten aus der 2. Versuchsreihe sind homogene Legierungen. In Tab. 3.1 sind noch einmal alle wichtigen Parameter der durchgeführten Tests zusammengefasst.

\begin{tabular}{|l|c|c|c|c|}
\hline & Probe 1 & Probe 2 & Simulat. 1 & Simulat. 2 \\
\hline Ag-Gehalt [At.\%] & 2,8 & 3,0 & 2,1 & 2,1 \\
\hline Ag-Gehalt im Test ${ }^{8}$ [At.\%] & 2,2 & 2,2 & 2,1 & 2,1 \\
\hline Stichprobengröße & 50 & 100 & 50 & 100 \\
\hline Stichprobenanzahl & 539 & 75 & 567 & 75 \\
\hline Freiheitsgrad & 5 & 5 & 5 & 5 \\
\hline kritischer $\chi^{2}$-Wert $(a=0,05)^{9}$ & 12,6 & 12,6 & 12,6 & 12,6 \\
\hline ermittelter $\chi^{2}$-Wert & 3,6 & 4,7 & 6,6 & 4,5 \\
\hline Homogenität & bestätigt & bestätigt & bestätigt & bestätigt \\
\hline
\end{tabular}

Tab. 3.1 Parameter und Ergebnisse der $\chi^{2}$-Anpassungstests zur Kontrolle der Homogenität der Fe-AgLegierungen.

\subsection{Sputtereffekte bei der gepulsten Laserdeposition}

Die Ergebnisse aus den vorangegangenen Teilkapiteln zeigten, dass die auf W-Substratspitzen deponierten Fe-Ag-Schichten sich hinsichtlich ihrer Struktur und Zusammensetzung gänzlich anders als die Referenzschichten auf planaren Substraten darstellten. Dadurch bedingt konnte die ursprüngliche Intention, ein Gesamtbild der weiteren nanostrukturellen Entwicklung in Verbindung mit den Untersuchungen an planaren Schichten $\mathrm{zu}$ zeichnen, nicht weiterverfolgt werden. Da die Herstellung der übersättigten Legierungen an die spezielle Art der Herstellung mittels PLD gekoppelt war, wurde vielmehr der Versuch unternommen, die Ursachen für die unterschiedlichen Resultate bei der Deposition besser zu verstehen. Diese mussten in den speziellen

\footnotetext{
${ }^{8}$ Nur exakt positionierbare Atome wurden berücksichtigt. Dies wirkte sich präferentiell auf den Ag-Gehalt aus, s. Text und Kap. 2.4.4.

${ }^{9} a$ bezeichnet das Signifikanzniveau
} 
Bedingungen der Deposition auf Spitzen im Vergleich zu planaren Schichten begründet sein. Neuste Untersuchungen an planaren Schichten [FÄHL98] zeigten, dass präferentielle Sputtereffekte dabei eine nicht unbedeutende Rolle spielen könnten. Insbesondere wurde durch Messungen von Depositionsraten in Abhängigkeit der Depositionswinkel [STUR99] gefunden, dass unter den Bedingungen der PLD die Sputterausbeuten für die Deposition von Eisen auf Eisen $\mathrm{Y}_{\mathrm{Fe}-\mathrm{Fe}}=0,13$ und für Silber auf Silber $\mathrm{Y}_{\mathrm{Ag}-\mathrm{Ag}}=0,55$ betrugen und damit nicht $\mathrm{zu}$ vernachlässigen waren. Außerdem zeigte sich damit, dass Silber erwartungsgemäß eine deutlich höhere Ausbeute aufwies als Eisen. ${ }^{10}$

Bei der Deposition auf TAP-Spitzen sind aufgrund der halbsphärischen Form der Spitzenapexes alle Depositionswinkel vertreten. Von besonderem Interesse war es daher, abzuschätzen, wie groß präferentielle Sputtereffekte bei der Deposition von Legierungen sein können. Es wurden deshalb TRIM-Simulationen durchgeführt und verschiedene Sputterausbeuten dabei bestimmt. Die Simulations-Software und die verwendeten Parameter der Simulation sind in Anhang A genauer dargestellt.

Die kinetischen Energien der Ionen im Experiment unterliegen einer Verteilung, die aus einer modifizierten Maxwell-Boltzmann Verteilung der Teilchendichten im PLDPlasma abgeleitet werden kann [KOOL92, FÄHL98]. Das Maximum dieser Verteilung liegt typischerweise in der Nähe von $100 \mathrm{eV}$. Für die TRIM-Simulationen wurden diskrete Ionenenergien verwendet. ${ }^{11}$ Sie wurden durch die Berechnung der gewichteten Mittelwerte der Energieverteilungen von Fe- und Ag-Ionen, die an der PLD-Anlage unter ähnlichen Bedingungen gewonnen wurden, wie sie bei der Deposition herrschten [FÄHL98, KREB99], abgeschätzt. Für Eisen wurde eine mittlere kinetische Energie von $136 \mathrm{eV}$, für Silber von $144 \mathrm{eV}$ berechnet. Auf Basis dieser Werte wurden dann mittels TRIM Sputterausbeuten $Y$ in Abhängigkeit des Depositionswinkels ${ }^{12} \varphi$ für die Deposition von Eisen auf Eisen, Eisen auf Silber, Silber auf Silber und Silber auf Eisen bestimmt. Als Sputterausbeute wurde dabei das Verhälnis der Anzahlen von aus der Oberfläche gesputterten Atomen zu ankommenden Ionen definiert. Die Simulationen zeigten dabei, dass der Anteil an rückgestreuten Ionen $\left(\mathrm{Y}_{\mathrm{bs}}\right)$, die demzufolge nicht auf dem Substrat deponiert wurden, zum Teil erheblich war (mehr als 50\%). Um diesen Effekt zu berücksichtigen wurde zusätzlich eine effektive Sputterausbeute $Y_{\text {eff }}$ bestimmt. Sie wurde definiert als Verhältnis der Anzahlen aus der Oberfläche gesputterter Atome $\mathrm{zu}$ deponierten (nicht rückgestreuten) Ionen $\left(\mathrm{Y}_{\mathrm{eff}}=\mathrm{Y} /\left(1-\mathrm{Y}_{\mathrm{bs}}\right)\right)$. In Abb. 3.8 sind die effektiven Sputterausbeuten in Abhängigkeit der Depositionswinkel graphisch dargestellt. Die Daten sind auch in tabellarischer Form in Anhang A aufgelistet.

Die berechnete effektive Sputterausbeute bei senkrechtem Ionenstrahleinfall für die Deposition von Silber auf Silber beträgt $Y_{\text {eff,Ag-Ag }}=0,49$ und ist damit doppelt so groß, wie die von Eisen auf Eisen $\mathrm{Y}_{\text {eff,Fe-Fe }}=0,25$. Interessant ist auch, dass die effektive Sputterausbeute von Eisen auf Silber erheblich größer $\left(\mathrm{Y}_{\text {eff,Fe-Ag }}=0,91\right)$ und umgekehrt die von Silber auf Eisen erheblich kleiner $\left(\mathrm{Y}_{\text {eff,Ag-Fe }}=0,07\right)$ ist, als die jeweils zuvor genannten Größen. Jedes deponierte Fe-Ion sputtert also 0,91 Ag-Atome aus Silber wieder heraus,

10 Als Maß für die Leichtigkeit ein Atom aus einer Oberfläche herauszuschlagen kann die Sublimationsenthalpie dienen. Sie beträgt für Eisen 414 kJ/mol und für Silber $285 \mathrm{~kJ} / \mathrm{mol}$.

11 TRIM-Simulationen mit variierten Ionenenergien zeigten, dass die resultierende, mit der Energieverteilung gewichtete mittlere Sputterausbeute sich kaum unterschied von der, die mit Hilfe des gewichteten Mittelwertes der kinetischen Energien bestimmt wurde.

${ }^{12}$ Winkel zwischen Oberflächennormale des Substrates und der Richtung des Ionenstrahls. 
während ein deponiertes Ag-Ion quasi kein $(0,07)$ Eisen zu sputtern vermag. Die kinetischen Energien von Eisen und Silber waren dabei nahezu identisch (136 eV und $144 \mathrm{eV}$ ). Hier kam offensichtlich ein ballistischer Effekt zum Tragen. Ein leichtes Element (Fe) kann durch einen (oder mehrere) Stoßprozess(e) mit einem schwereren Element (Ag) leicht einen rückwärtig, in Richtung Oberfläche gerichteten Impuls erhalten, der für das Sputtern des Targetelements (Ag) notwendig ist. Für den umgekehrten Fall ist dies erheblich schwieriger.

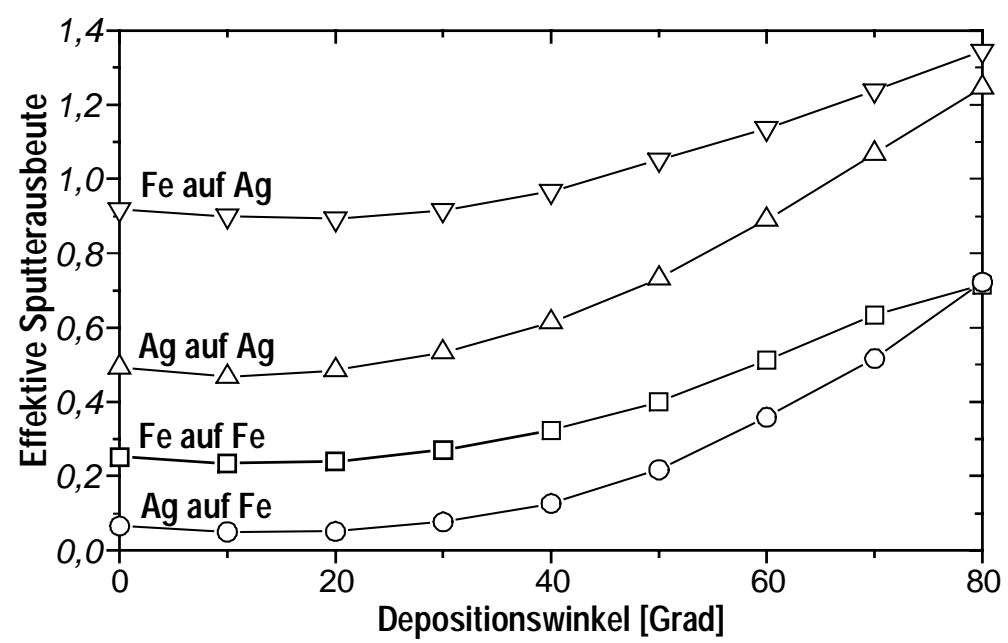

Abb. 3.8 Effektive Sputterausbeuten (s. Text) in Abhängigkeit von Depositionswinkeln, bestimmt mittels TRIM-Simulationen.

Abb. 3.8 zeigt ferner, dass die effektiven Sputterausbeuten mit zunehmendem Depositionswinkel auch deutlich ansteigen. Beispielsweise nimmt bei Silber auf Silber $Y_{\text {eff }}$ bis $\varphi=60^{\circ}$ um 0,4 auf 0,89 zu. Bei Eisen auf Eisen beträgt in demselben Fall die Zunahme 0,26 auf 0,51. Die Änderungen der effektien Sputterausbeuten haben einen erheblichen Einfluss auf die Depositionsraten. Dies umso mehr je größer die Ausbeute bereits bei $\varphi=0^{\circ}$ ist. Denn die Menge an deponierten Atomen ist maßgeblich bestimmt durch den Faktor (1-Y $\left.Y_{\text {eff }}\right)$ - einem deponierten Ion stehen $Y_{\text {eff }}$ gesputterte entgegen. Berücksichtigt man noch den Anteil rückgestreuter Ionen $Y_{b s}$ - für Eisen auf Eisen und Silber auf Silber sind die Werte nahezu identisch -, so ergibt sich für die Depositionsrate:

$$
R(\varphi)=1-Y-Y_{b s}=\left(1-Y_{e f f}\right) \cdot\left(1-Y_{b s}\right) .
$$

Für die Deposition von Eisen auf Eisen und Silber auf Silber ergeben sich so auf Basis der TRIM-Daten und Gleichung 3.1 die Depositionsraten in Abhängigkeit vom Depositionswinkel. In Abb. 3.9 ist dieser Zusammenhang graphisch dargestellt. $R(\varphi=0)$ ist dabei normiert auf den Wert 1. Deutlich sichtbar ist, dass die Rate für Silber schneller abnimmt als die von Eisen. Bei $\varphi=60^{\circ}$ ist die Silber-Rate bereits auf 0,18 abgefallen um kurz dahinter sogar negativ zu werden, während die Eisenrate bei $\varphi=60^{\circ}$ noch 0,54 beträgt. 


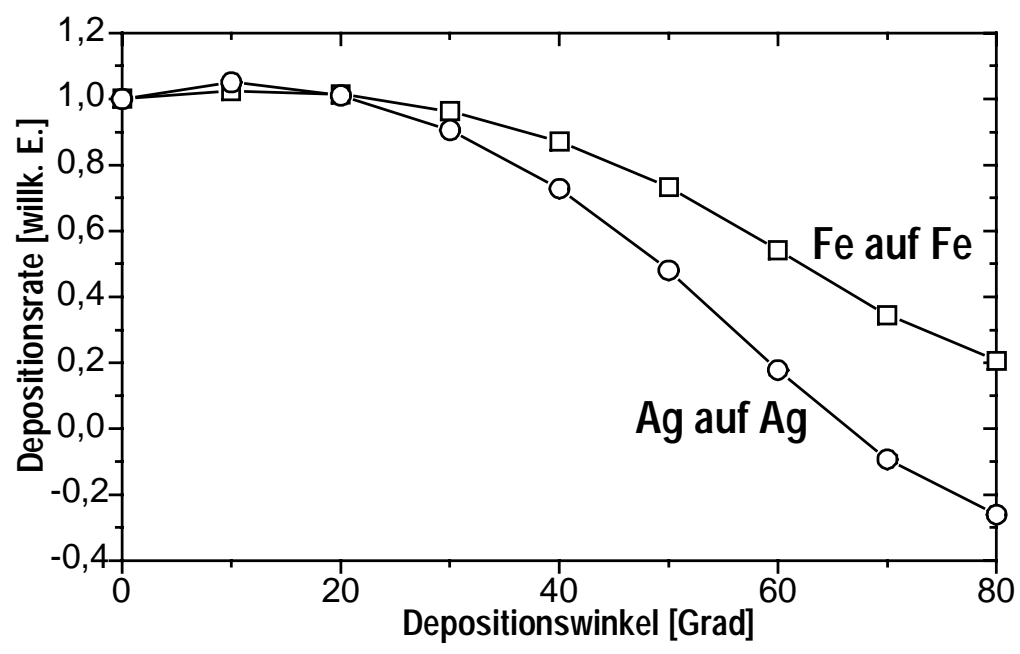

Abb. 3.9 Depositionsraten berechnet auf Basis von TRIM-Simulationen für die Deposition von $136 \mathrm{eV}$ Eisen auf Eisen und $144 \mathrm{eV}$ Silber auf Silber.

Die Sputterausbeuten von Eisen und Silber sind wesentlich von den OberflächenBindungsenergien $\mathrm{E}_{\mathrm{s}}$ abhängig. Diese wurden mit $\mathrm{E}_{\mathrm{s}}(\mathrm{Fe})=4,34 \mathrm{eV}$ und $\mathrm{E}_{\mathrm{s}}(\mathrm{Ag})=2,97$ eV (s. Anhang A), was den Sublimationsenthalpien entspricht, angesetzt. Für Fe-AgLegierungen sind die entsprechenden Werte nicht bekannt. Als erste Näherung sei hier deshalb grob abgeschätzt, dass sich die Oberflächen-Bindungsenergien in einer Legierung aus den genannten Randwerten der reinen Komponenten abzüglich des jeweiligen chemischen Potentials ergeben. Auf der Eisen-reichen Seite wird für Silber nach [BOER88] eine partielle Mischungsenthalpie von $123 \mathrm{~kJ} / \mathrm{mol}$ angegeben. Gemäß dem in [BOER88-Kapitel 2] beschriebenen Modell und der Annahme einer idealen Mischungsentropie kann das chemische Potential von Silber in Eisen bei Temperaturen von $\mathrm{T}=300 \mathrm{~K}$ mit $80 \mathrm{~kJ} / \mathrm{mol}$ abgeschätzt werden. Das entspricht ca. 0,8 eV pro Atom Silber. Unter der Annahme, dass für in Eisen gelöstes Silber eine OberflächenBindungsenergie von abgeschätzt $\mathrm{E}_{\mathrm{s}}=2,97-0,8=2,17 \mathrm{eV}$ vorliegt und der Wert für Eisen unverändert bleibt, ergibt sich aus TRIM-Simulationen der Deposition von Eisen auf Fe-Ag-Legierungen mit 1, 5 und 10 At.\% Ag eine effektive Sputterausbeute $\mathrm{Y}_{\mathrm{eff}}\left(\varphi=0^{\circ}\right)$ von 0,61 bzw. 0,67 und 0,68. Für Eisen ergeben sich Werte von 0,25 bzw. 0,27 und 0,29. Es wird deutlich, dass auch im Fall einer Eisen-reichen Legierung bei der Deposition von Eisen eine große Differenz zwischen den Sputterausbeuten von EisenAtomen und Silber-Atomen vorliegt.

\subsection{Diskussion der Ergebnisse}

In dieser Arbeit wurden Schichten von nanokristallinen Fe-Ag-Legierungen auf WSubstratspitzen mittels PLD hergestellt. Es sollten ihre Strukturen und chemischen Zusammensetzungen auf kleinster möglicher Längenskala mit der Atomsondentechnik untersucht werden. Dabei sollten die ,wie hergestellten“ Zustände sowie Zustände nach thermischer Behandlung charakterisiert werden, um ihre Mikrostruktur und ihre Entwicklung bei der Entmischung zu verfolgen und die Ergebnisse mit den XRD und TEM-Untersuchungen an planaren Schichten, die parallel in einer Arbeit von M. Störmer durchgeführt wurden [STÖR98], in einen gemeinsamen Zusammenhang zu stellen. Die geringen möglichen Schichtdicken bei der PLD verhinderten die Präparation der Atomsonden-Spitzen direkt aus den planaren Schichten heraus. Sie wurden vielmehr separat auf Wolfram-Substratspitzen aufgebracht. Um einen unmittelbaren Vergleich zu 
planaren Schichten zu ermöglichen wurden parallel jeweils planare Referenzsubstrate unter denselben Bedingungen wie die Spitzen beschichtet.

In den vorhergehenden Teilkapiteln wurden die experimentellen Ergebnisse der Untersuchungen an Fe-Ag-Schichten auf W-Spitzen vorgestellt. Dabei zeigen bereits die TEM-Untersuchungen, dass die auf Spitzen deponierten Schichten sich deutlich von den auf planaren Schichten unterscheiden. Die Schichtdicken fallen erheblich geringer aus, als im Vergleich zu den planaren Referenzen. Nur die Hälfte bis ein Drittel wird deponiert. Dies deutet auf einen zusätzlichen Abtrag durch Sputtern hin. Nach [NAST96-Kap.9] können die Schwellwerte der kinetischen Energien $E_{\text {th }}$, bei denen Sputtern einsetzt, gemäß folgendem Ansatz berechnet werden:

$$
E_{t h}= \begin{cases}\left(\frac{4}{3}\right)^{6} \frac{\left(M_{1}+M_{2}\right)^{2}}{4 M_{1} M_{2}} \cdot E_{s} & , M_{1} \geq M_{2} \\ \left(\frac{2 M_{1}+2 M_{2}}{M_{1}+2 M_{2}}\right)^{6} \frac{\left(M_{1}+M_{2}\right)^{2}}{4 M_{1} M_{2}} \cdot E_{s} & , M_{1}<M_{2}\end{cases}
$$

Dabei ist $\mathrm{E}_{\mathrm{s}}$ die Oberflächen-Bindungsenergie, s. Kap. 3.4, $\mathrm{M}_{1}$ die Masse der Ionen und $\mathrm{M}_{2}$ die Masse der Atome in der Schicht. Daraus ergeben sich Schwellwerte von 10 bis $27 \mathrm{eV}\left(\mathrm{E}_{\text {th }, \mathrm{Fe} \rightarrow \mathrm{Fe}}=27 \mathrm{eV} ; \mathrm{E}_{\text {th }, \mathrm{Ag} \rightarrow \mathrm{Fe}}=27 \mathrm{eV} ; \mathrm{E}_{\text {th }, \mathrm{Ag} \rightarrow \mathrm{Ag}}=19 \mathrm{eV} ; \mathrm{E}_{\text {th }, \mathrm{Ag} \rightarrow \mathrm{Fe}}=10 \mathrm{eV}\right)$. Die kinetischen Energien der ablatierten Ionen sind mit mehr als $100 \mathrm{eV}$ also ausreichend groß, um das Sputtern von der Schicht hervorzurufen.

Ähnliche Effekte sind auch bei anderen Autoren bereits beobachtet worden, wenn auch in etwas geringerem Umfang. Von Al-Kassab et al. wird berichtet, dass auch bei der Argon-Sputter-Deposition (von Palladium und Kupfer) auf W-Spitzen weniger als 60\% der Dicke der planaren Referenzschichten auf den Spitzen gefunden werden [ALKA96]. Hierbei liegen die kinetischen Energien nach Angabe der Autoren auch bei ca. $100 \mathrm{eV}$. Die Ausbildung einer charakteristischen Kappenform der Schichten, wie sie hier jedoch zusätzlich beobachtet wird, zeigt sich allerdings bei der Sputter-Deposition nicht. Bei der PLD wird sie aber auch bei anderen Materialien gefunden, beispielsweise bei der Depositon von Palladium-Niob-Schichtungen [KEST99]. Auch hier kann ein Zusammenhang mit Sputtereffekten vermutet werden. En détail verstanden ist dieser Effekt bisher jedoch nicht.

Die Korngrößen, die sich aus den TEM- und den FIM-Untersuchungen ergeben, überraschen zunächst sehr. Da sind einerseits die großen (auf der FIM-Längenskala) einkristallinen Kappen der Größenordnung $100 \mathrm{~nm}$ aus der ersten Versuchsreihe, andererseits Korngrößen von ca. $15 \mathrm{~nm}$ bei der zweiten. Die planaren Referenzschichten weisen dabei Ag-Gehalte von um 8 At.\% bzw. über 20 At.\% auf. Bei diesen hohen Konzentrationen werden nach [STÖR95] erheblich kleinere Korngrößen (10 bzw. 5 nm) in planaren Schichten gefunden, als auf den Spitzen beobachtet. Dieser Umstand deutet bereits darauf hin, dass die mittleren Ag-Konzentrationen der Schichten auf den Spitzen von denen auf planaren Substraten abweichen. Dennoch sind die Korngrößen in jedem Fall ungewöhnlich groß im Vergleich zu dem was allgemein bei PLD-Schichten gefunden wird. Selbst bei reinen Elementen sind diese typischerweise nicht größer als 10 bis $20 \mathrm{~nm}$ [KREB95].

Die im FIM beobachteten gut entwickelten und klar strukturierten Polfiguren - bei der ersten Versuchsreihe - zeigen ihrerseits, dass der Ag-Gehalt in den Schichten nicht allzu groß sein kann. Es ist allgemein bekannt, dass Legierungen mit substitutioneller Lösung bei größeren Konzentrationen der Legierung - ausgenommen sind hier Ordnungsphasen 
- keine oder lediglich nur schwache Strukturen im FIM-Bild zeigen [MILL96-Kap. 3.5]. Die Ursache dafür ist darin begrünet, dass die Feldverdampfung an der Spitzenoberfläche durch die unterschiedlichen Legierungskomponenten nicht mehr sehr gleichmäßig geordnet verläuft, und dadurch die ansonsten regelmäßigen Terassenstrukturen unregelmäßiger und folglich die FIM-Abbildungen mehr und mehr unstrukturierter werden. Die FIM-Bilder aus der zweiten Versuchsreihe mit nominell höheren AgGehalten, die deutlich unstrukturierter sind als letztere, deuten deshalb auch eindeutig darauf hin, dass die Ag-Gehalte in diesem Fall größer sind.

Eine geringere Ag-Konzentration als nominell angenommen erklärte auch die Epitaxie zum Wolfram-Substrat, die bei den Schichten der ersten Versuchsreihe nachgewiesen wurde. Für Schichten reiner Elemente ist das epitaktisches Wachstum auf Spitzen ein bekanntes Phänomen [MILL96]. FIM-Untersuchungen an auf W-Substratspitzen thermisch aufgedampften Fe-Schichten von Morikawa et al. [MORI78] zeigten auch ein epitaktisches Wachstum von Eisen auf Wolfram. Die Substrattemperaturen ${ }^{13}$ lagen hierbei mit $100{ }^{\circ} \mathrm{C}$ nicht weit entfernt von Raumtemperatur. Sie sollten daher mit den Substrattemperaturen bei der PLD vergleichbar sein.

Das von den TEM- und FIM-Untersuchungen gezeichnete Bild wird klar bestätigt durch die AP- und TAP-Analysen. Für die Schichten der ersten Versuchsreihe wird eine mittlere Ag-Konzentration von 1,1 At.\% gemessen. Dieser Wert steht in deutlicher Diskrepanz zu den nominellen Konzentrationen der planaren Schichten von ca. 8 At.\%. Bei den Schichten der zweiten Versuchsreihe, die mit der TAP untersucht wurden, ist das Missverhältnis in etwa dasselbe. Die mittleren Ag-Konzentrationen von ca. 3 At.\% liegen um einen Faktor 7 bis 9 unterhalb der nominellen Referenz. Die Fe-Ag-Schichten auf den W-Spitzen weisen deshalb, verständlicherweise, deutlich andere Strukturen und Korngrößen auf, als dies im Vergleich mit den planaren Schichten zu erwarten gewesen wäre.

Die mit AP und TAP gemessenen Ag-Konzentrationen von bis zu 3 At.\% erreichen zwar nicht die Übersättigung, die bei den planaren Schichten deponiert werden konnte, liegen aber dennoch weit oberhalb der Löslichkeitsgrenze für Silber in Eisen im thermodynamischen Gleichgewicht, die selbst bei hohen Temperaturen verschwindend gering ist - 0,022 At. $\%$ bei $\mathrm{T}=1398{ }^{\circ} \mathrm{C}$ [WRIE73, MASS90]. Die statistische Auswertung der AP- und TAP-Analysen zeigt eindeutig, dass das Silber bis zu 3 At.\% homogen in der Eisen-Matrix gelöst ist. Damit ist es erstmals gelungen, unmittelbar nachzuweisen, dass mit Hilfe der PLD eine stark entmischende Legierung mit einer großen positiven Mischungsenthalpie weit über ihre Löslichkeitsgrenze hinaus übersättigt werden kann. Mit der Einschränkung auf Konzentrationen von bis zu 3 At.\% $\mathrm{Ag}$ können die Interpretationen der XRD-Ergebnisse von Störmer und Krebs [STÖR95] hinsichtlich der homogenen Übersättigung von Fe-Ag-Legierungen damit bestätigt werden. Die Autoren konnten zeigen, dass bis zu Ag-Gehalten von 14 At.\% keine Peaks der reinen Elemente, sondern ausschließlich Legierungspeaks auftreten, woraus auf die Homogenität geschossen wurde.

Die Unterschiede in den Ag-Gehalten zwischen planaren Schichten und Schichten auf Spitzen zeigen, dass während des Depositionsprozesses bei der PLD ein erheblicher präferentieller Abtrag von Silber stattgefunden haben muss. Da die planaren Referenzproben parallel zu den Spitzen hergestellt wurden, kann dies lediglich in dem

\footnotetext{
${ }^{13}$ Von Morikawa et al. wurden Untersuchungen bei Substrattemperaturen von $-198{ }^{\circ} \mathrm{C}$ und zwischen 100 und $800{ }^{\circ} \mathrm{C}$ durchgeführt.
} 
Unterschied der Substratgeometrien begründet sein. Bei der Herstellung planarer Schichten erfolgt die Deposition unter einem wohldefinierten Winkel. Bei den Spitzen hingegen überstreichen die Depositionswinkel, bedingt durch die halbsphärische Form der Spitzenapexes, große Raumwinkelbereiche. Winkelabhängige präferentielle Sputtereffekte könnten daher die Ursache für den vermehrten Abtrag von Silber sein. Neue Untersuchungen von Fähler an planaren Fe-Ag-Schichten [FÄHL98] zeigen, dass präferentielles Sputtern bei der PLD existiert. Auch die hier vorgestellten TRIMSimulationen geben einen Hinweis darauf, wie groß diese Effekte sein können. Die aus den Simulationen bestimmten (effektiven) Sputterausbeuten bei senkrechtem Einfallswinkel sind in guter Übereinstimmung mit neuen experimentellen Ergebnissen von Sturm et al. [STUR99]. Bei Silber auf Silber wird dort eine Sputterausbeute von 0,55 bestimmt. Die TRIM-Simulationen ergeben einen Wert von 0,49. Die aus den TRIMDaten abgeleiteten winkelabhängigen Depositionsraten zeigen ferner, dass ab Winkeln von $40^{\circ}$ deutlich weniger Silber deponiert wird als Eisen. Desweiteren ist die effektive Sputterausbeute bei der Deposition von Eisen auf Silber noch erheblich größer $(0,92)$ als bei Silber auf Silber, während die von Silber auf Eisen deutlich kleiner ist $(0,07)$ als die von Eisen auf Eisen. Dieser qualitative Zusammenhang wurde auch experimentell von Sturm et al. [STUR99] an Grenzflächen zwischen Eisen und Silber bei der Deposition von Fe-Ag-Schichtungen beobachtet. ${ }^{14}$ Als Ursache dieses Zusammenhanges wird ein rein ballistischer Effekt vermutet. Bei insgesamt nur wenigen Stoßprozessen pro Ion treten rückwärtig gerichtete Impulse, die das Sputtern erst ermöglichen, abhängig von den Massenverhältnissen mit unterschiedlichen Wahrscheinlichkeiten auf.

Die TRIM-Simulationen mit reinen Elementen liefern natürlich nur Hinweise bezüglich der Größe von Sputtereffekten und deren Winkelabhängigkeiten in übersättigten Legierungen. Dennoch wird deutlich, dass die unterschiedlichen (partiellen) Sputterausbeuten eine entscheidende Rolle spielen. Sie sind wesentlich abhängig von den Oberflächen-Bindungsenergien der einzelnen Komponenten. In Kap. 3.4 wurde anhand der Freien Energien des Legierungssystems Eisen-Silber der Einfluss der Mischung von Eisen und Silber auf die Oberflächen-Bindungsenergien abgeschätzt. Die auf der Basis dieser Werte durchgeführten TRIM-Simulationen zeigen, dass auch bei übersättigten Legierungen eine große Differenz zwischen den partiellen Sputterausbeuten vorliegen kann. Ein weiterer Aspekt, der bisher nicht berücksichtigt wurde, betrifft den Einfluss von neutralen niederenergetischen Atomen. Bei der PLD besteht ein nicht genau bestimmter Anteil des deponierten Materials nicht aus hochenergetischen Ionen sondern aus neutralen Atomen, deren kinetische Energie schätzungsweise 5-10 eV beträgt. Ihr Anteil wird auf 50 bis 100\% [KREB97] geschätzt. Diese Atome tragen nicht zum Sputtern bei. Sie deponieren jedoch, aufgrund der geringen kinetischen Energie, direkt auf der Oberfläche. Da die Elemente Eisen und Silber abwechselnd deponiert werden, kann dies auch einen Einfluss auf die partiellen Sputterausbeuten haben. Die gesamte Problematik kann jedoch in ihrer Komplexität hier nicht aufgelöst werden. Es bleibt festzustellen, dass die TRIM-Simulationen Hinweise dafür geben, dass vermutlich präferentielles Sputtern für den zusätzlichen Ag-Abtrag auf Spitzen im Vergleich zu planaren Schichten verantwortlich ist.

Die Untersuchungen an den Schichten auf Spitzen zeigen, dass bei der PLD ein Eins-zuEins-Übertrag von Target-Material auf das Substrat, wie er als allgemein charakteristisch für die Methode gilt [KREB97], in diesem Fall für Eisen-Silber keine Gültigkeit

\footnotetext{
${ }^{14}$ Ein quantitativer Vergleich der experimentellen Daten mit den TRIM-Daten kann prinzipiell auch erfolgen, soll hier aber nicht weiter behandelt werden.
} 
hat. Es ist anzunehmen, dass dies auch für andere Legierungssysteme, wenn diese auf gekrümmten Oberflächen deponiert werden sollen, gilt. Die deponierten Strukturen sind dann über die Oberläche hinweg variierend und relativ undefiniert. Eine weitere Untersuchung der Mikrostruktur, sowie thermische Behandlungen der Schichten auf Spitzen wurden deshalb nicht durchgeführt.

Für weitere Untersuchungen an mit PLD deponierten Fe-Ag-Schichten könnte eventuell versucht werden, insbesondere den hochenergetischen Anteil der Ionen bei der PLD zu verringern, um die Sputtereffekte gering zu halten. Allerdings könnte dadurch auch die Homogenität der Legierungen verloren gehen, da die Ionen gerade auch für die Durchmischung sorgen. Entsprechende PLD-Experimente unter Ar-Gas-Atmosphäre [STUR99] zeigten, dass bei Ar-Partialdrücken von $4 \times 10^{-2} \mathrm{hPa}$ die Energien der deponierten Ionen reduziert werden konnten und auch die Sputtereffekte deutlich verringert waren. Allerdings zeigte sich bei der Deposition auf W-Spitzen ein neuer unerwarteter Effekt: es war ein extremes Schichtwachstum zu beobachten, dass weit über das der planaren Schichten hinausging. Die resultierenden Spitzen waren daher für weitere Untersuchungen ungeeignet. Die Ergebnisse zur Deposition unter Ar-Gas sind für den interessierten Leser in Anhang B zusammengefasst.

Die AP- und TAP-Untersuchungen zeigen evident, dass eine homogene starke Übersättigung der Legierungen möglich ist. Hinsichtlich der Entwicklung der Mikrostruktur der (nanokristallinen) übersättigten Fe-Ag-Legierungen können die Untersuchungen an planaren Schichten von Störmer [STÖR98], aufgrund der fehlenden Vergleichbarkeit, durch TAP-Analysen aber nicht erweitert werden. Die planaren Schichten zeigen eine höchst interessante thermische Stabilität. Voraussetzung dafür ist, dass im „wie hergestellten“ Zustand keine Ag-Anreicherungen vorliegen, die Proben also homogen sind. Dies ist nach den Ergebnissen dieser Arbeit offensichtlich möglich. Störmer konnte zeigen, dass die Korngröße durch Auslagerung bei $400{ }^{\circ} \mathrm{C}$ über ca. $24 \mathrm{~h}$ von anfangs 8,5 $\mathrm{nm}$ auf $15 \mathrm{~nm}$ ansteigt und sich dann stabilisiert. Als Ursache wird eine Segregation des Silbers in den Korngrenzen der nanokristallinen Struktur vermutet. Im Einklang damit wird in XRD-Messungen von Fe-9 At.\% Ag-Legierungen bei Temperung bis zu $620{ }^{\circ} \mathrm{C}$ kein Silber-Reflex beobachtet, obwohl sich die Gitterkonstante in Richtung der des reinen Eisens verschiebt. Die entsprechenden Diffraktogramme sind in Abb. 3.10 dargestellt. Störmer geht davon aus, dass das Silber in die Korngrenzen diffundiert ist. Bei einem Kornwachstum von 8,5 auf $15 \mathrm{~nm}$ kann aber auch allein durch die Bewegung der Korngrenze das Silber aus den „überlaufenen“ Körnern darin „,aufgesammelt" worden sein. Bei einem Korndurchmesser von $\mathrm{D}=15 \mathrm{~nm}$ beträgt die Korngrenzfläche pro Mol Fe-9 At.\% Ag-Legierung nach Gleichung 4.2 ca. $1500 \mathrm{~m}^{2}$. Unter der Annahme, dass alles Silber in den Korngrenzen segregiert wäre, ergibt sich damit ein maximaler Korngrenzen-Exzess von $\Gamma_{\max }=3,7 \times 10^{19} \mathrm{~m}^{-2}$, was ca. 1,3 Monolagen einer (111)-fcc-Silber-Ebene entspricht. Es ist davon auszugehen, dass AgAtome in einer Fe-Korngrenze, aufgrund der gelockerten Struktur derselben, deutlich geringere chemische Potentiale aufweisen, als Ag-Atome innerhalb einer bcc-Fe-Matrix im Korninneren. Mit einer Segregation in der genannten Größenordnung wäre daher eine erhebliche Absenkung der Korngrenzenenergie verbunden. Da mit der berechneten Ag-Belegung der Korngrenzen diese vermutlich gesättigt sind, müsste bei weiterem Kornwachstum Silber wieder im Korn gelöst werden, was energetisch ungünstig ist. Allein aus Sicht der verringerten thermodynamisch treibenden Kraft für das Kornwachstum (s. auch Gl. 4.3) wäre so die thermische Stabilität begründbar. Da über die Beweglichkeit von Silber in Eisen nichts bekannt ist, kann über den zusätzlichen Einfluss einer eventuellen kinetischen Hemmung des Kornwachstums nichts ausgesagt 
werden. Unabhängig davon kann die durch die Segregation hervorgerufene thermische Stabilität der Kornstruktur erst dadurch aufgebrochen werden, dass Silber aus den Korngrenzen entfernt wird. Dies wäre durch die Bildung von Ag-Ausscheidungen möglich. Die Experimente von Störmer [STÖR98] liefern dazu allerdings keinen Anhaltspunkt. Glühungen bei ausreichend höheren Temperaturen wurden nicht durchgeführt.

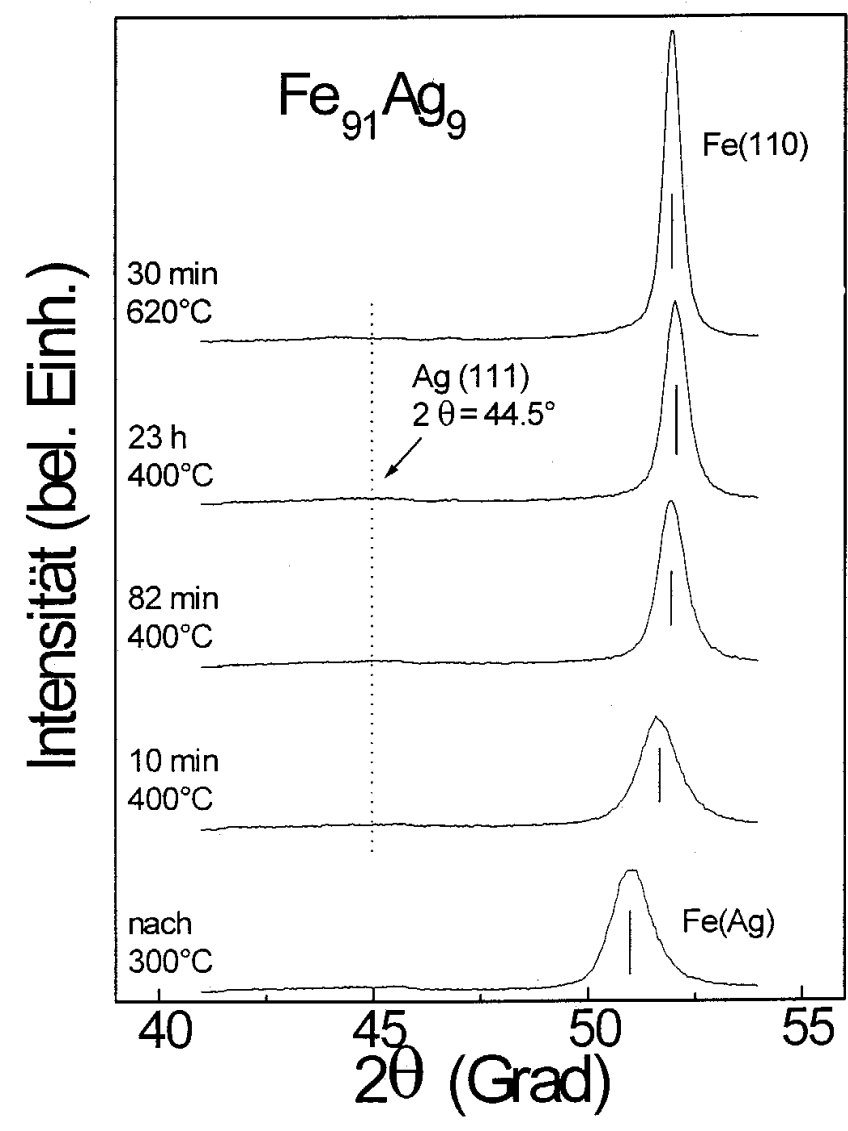

Abb. 3.10 Diffraktogramme einer als planare Schicht laserdeponierten Fe-9 At.\% Ag-Legierung, nach unterschiedlichen Wärmebehandlungen [STÖR98]. Bis zu $620{ }^{\circ} \mathrm{C}$ ist keine Ag-Peak zu beobachten.

Weitere Untersuchungen sind notwendig, um den genauen Mechanismus der Kornstruktur-Stabilisierung aufzuklären. 3D-Atomsonden-Analysen könnten prinzipiell wesentlich zur Auflösung der Zusammenhänge beitragen. Die Untersuchungen können jedoch nur dann erfolgreich sein, wenn die Proben unmittelbar aus den planaren Schichten heraus präpariert werden. In neuester Zeit sind Methoden entwickelt worden, die auf Elektronenstrahl-Lithographie und anschließendem Nachdünnen mit Hilfe von fokussierten Ionenstrahlen beruhen [LARS99], die es ermöglichen könnten auch planare FeAg-Schichten mit 3D-Atomsonden in Zukunft zu untersuchen. 


\section{Untersuchungen an Ni-P-Legierungen}

Das Verständnis der besonderen Eigenschaften von Ni-P-Legierungen nach der Herstellung und nach thermischer Behandlung setzt eine detaillierte Kenntnis der Mikrostrukturen und der P-Verteilungen voraus. Nach der Herstellung der Ni-P-Schichten, die - wie in Kap. 2.1 dargestellt - chemisch abgeschieden wurden, galt es daher zunächst die Mikrostruktur in diesem Abscheidezustand $\mathrm{zu}$ charakterisieren. Die wesentlichen Ergebnisse sind in Kap. 4.1 dargestellt. Es wurden dann gezielte Wärmebehandlungen an den Ni-P-Legierungen durchgeführt. Die verschiedenen Zustände, die sich dabei jeweils einstellten, wurden anschließend mit unterschiedlichen Methoden näher untersucht. Diese Untersuchungen werden in Kap. 4.2 vorgestellt. Die Diskussion der Ergebnisse erfolgt dann im Anschluss in Kap. 4.3.

\subsection{Mikrostruktur der Ni-P-Legierungen im Abscheidezustand}

\subsubsection{Mittlerer Phosphor-Gehalt}

Es wurde zunächst nach der Herstellung der Ni-P-Schichten der mittlere P-Gehalt bestimmt. Diese Analyse erfolgte mit Hilfe einer Elektronenstrahlmikrosonde (EMS). Die Schichten wurden dazu im Querschliff präpariert, s. Kap. 2.3. Dadurch war es möglich neben dem mittleren P-Gehalt auch den Verlauf der Konzentration über den Schichtquerschnitt zu kontrollieren. Bei allen Proben wurden keine nennenswerten Variationen der P-Konzentration auf der $\mu \mathrm{m}$-Skala beobachtet. Die mit der EMS bestimmten mittleren P-Gehalte für die vier unterschiedlichen Probenmaterialien sind in Tab. 4.1 aufgeführt. Diese Werte dienten in der gesamten Arbeit als nominelle Konzentrationen und wurden zur Bezeichnung der Probenmaterialien verwendet.

\begin{tabular}{|c|c|}
\hline Probenmaterial & $\begin{array}{c}\text { Mittlere P-Konzentration } \\
\text { [At. } \% \text { ] }\end{array}$ \\
\hline A & 3,6 \\
\hline B & 5,9 \\
\hline C & 6,4 \\
\hline D & 11,8 \\
\hline
\end{tabular}

Tab. 4.1 Mittlere Phosphor-Gehalte der Probenmaterialien aus einer Analyse mit der Elektronenstrahlmikrosonde.

\subsubsection{Korngrößen und Phasenzusammensetzung}

Zur Bestimmung der im Abscheidezustand in den Ni-P-Legierungen vorliegenden Phasen wurde die Röntgendiffraktometrie (XRD) eingesetzt. Die Diffraktogramme der vier Probenmaterialien sind in Abb. 4.1 zusammengefasst. Es wurden ausschließlich fcc-Nickel-Reflexe beobachtet. Peaks, die auf die Existenz einer, im thermodynamischen Gleichgewicht zu erwartenden, $\mathrm{Ni}_{3} \mathrm{P}$-Phase hingewiesen hätten, gab es keine. Die größte Intensität lieferten die (111)-Reflexe. Die korrespondierenden (200)-Reflexe waren dagegen relativ schwach ausgeprägt. Dies deutet darauf hin, dass eine Texturierung vorliegt. 


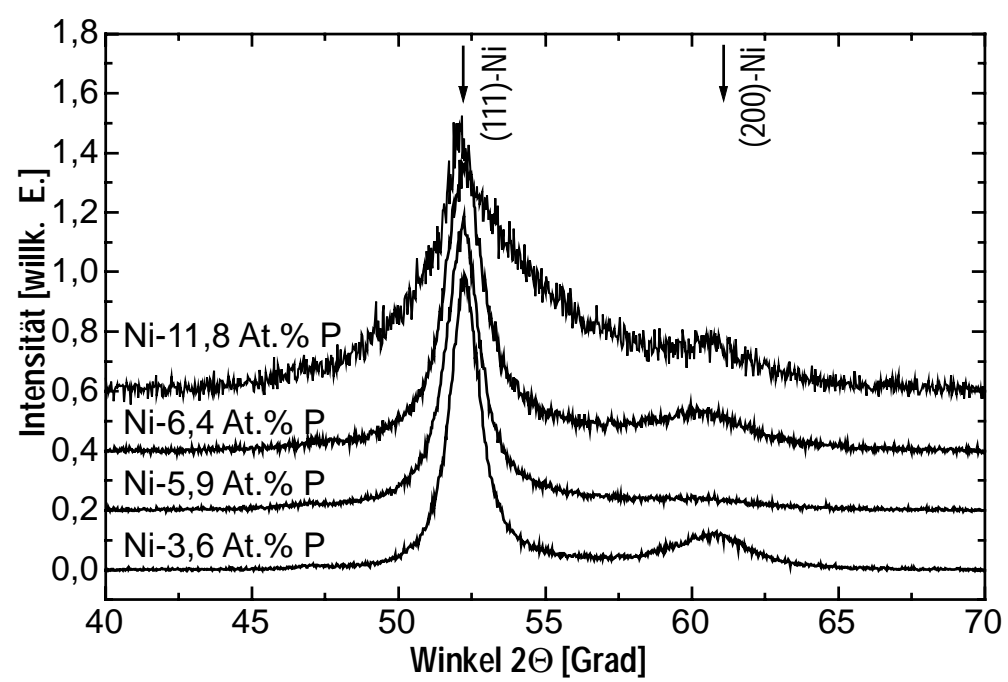

Abb. 4.1 Röntgendiffraktogramme der Ni-P-Legierungen im Abscheidezustand. Es sind ausschließlich fcc-Ni(P)-Reflexe zu beobachten.

Die Röntgenreflexe sind aufgrund der nanokristallinen Struktur der Legierungen erwartungsgemäß stark verbreitert. Anhand der Breite der (111)-Reflexe in den Diffraktogrammen wurden die mittleren Korndurchmesser mit Hilfe der ScherrerFormel, s. Gl. 2.6, bestimmt. Dabei ist anzumerken, dass der ohnehin schwache Reflex der Probe mit 11,8 At.\% P leicht asymmetrisch verläuft. Die Breite des Reflexes wurde aber auch hier anhand der Halbwertsbreite bestimmt. Die resultierenden Korngrößen in Abhängigkeit der mittleren P-Gehalte sind in Tab. 4.2 zusammengefasst. Die Lage der Reflexe lag zwischen 52,21 und 52,27 Grad und war damit im Rahmen der Messgenauigkeit konstant auf der Position des reinen (111)-Nickel-Reflexes.

\begin{tabular}{|c|c|}
\hline P-Gehalt [At.\%] & Korngröße [nm] \\
\hline 3,6 & 7,0 \\
\hline 5,9 & 6,1 \\
\hline 6,4 & 5,1 \\
\hline 11,8 & 2,9 \\
\hline
\end{tabular}

Tab. 4.2 Mittlere Korngrößen der NiP-Schichten im Abscheidezustand.

Die nanokristalline Struktur der Proben wurde auch mittels TEM überprüft. Die relativ geringe Auflösung des verwendeten Mikroskopes erlaubte allerdings keine Hochauflösung und keine dezidierte Abbildung einzelner Körner. Die Untersuchungen beschränkten sich daher auf die Überprüfung der Nanokristallinität. Die Proben zeigten alle erwartungsgemäß die nanokristalline Struktur. In Abb. 4.2 ist beispielhaft eine TEM-Hellfeldaufnahme (A) und eine TEM-Dunkelfeldaufnahme, (111)-Reflexe, (B) einer Ni-5,9 At.\% P-Legierung abgebildet. Einzelne hell abbildende Körner sind in Teil (B) sichtbar. Die Abbildung ist apparativ bedingt leicht verzerrt. 

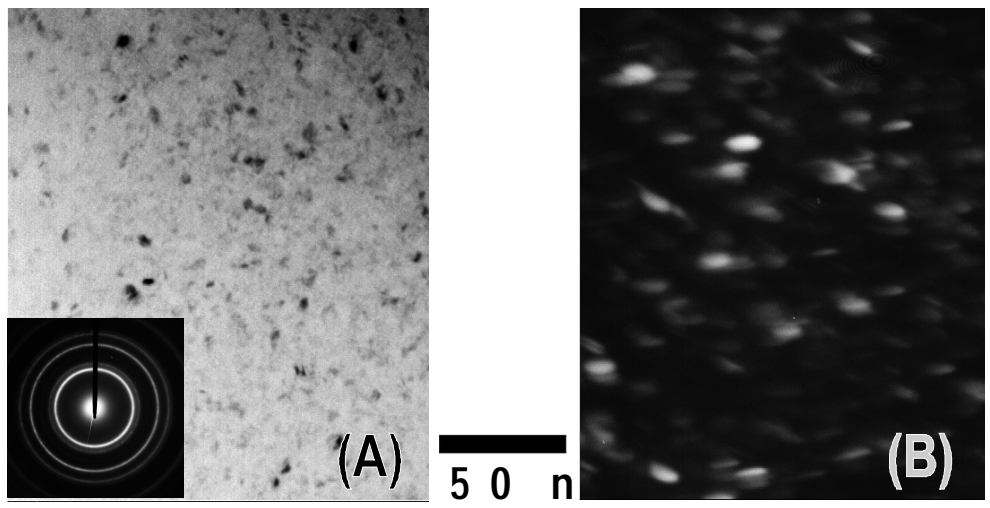

Abb. 4.2 TEM-Aufnahmen einer Ni-5,9 At.\% P-Legierung im Abscheidezustand. Im Teil (A) ist eine Hellfeldaufnahme mit Beugungsbild abgebildet. Im Teil (B) ist eine Dunkelfeldaufnahme dargestellt. Einzelne Körner sind hier sichtbar. Die Abbildung ist apparativ bedingt leicht verzerrt.

\subsubsection{Kristallographische Texturen}

Die in Kap. 4.1.2 vorgestellten röntgenographischen Untersuchungen zeigten, dass die kristallographischen Orientierungen der $\mathrm{Ni}(\mathrm{P})$-Körner nicht statistisch regellos sein konnten. Es wurden deshalb die (111)-Texturen der Ni-P-Schichten mit Hilfe des Röntgendiffraktometers untersucht. Die Ergebnisse sind in Abb. 4.3 in Form von Höhenliniendiagrammen (Polfiguren) dargestellt.

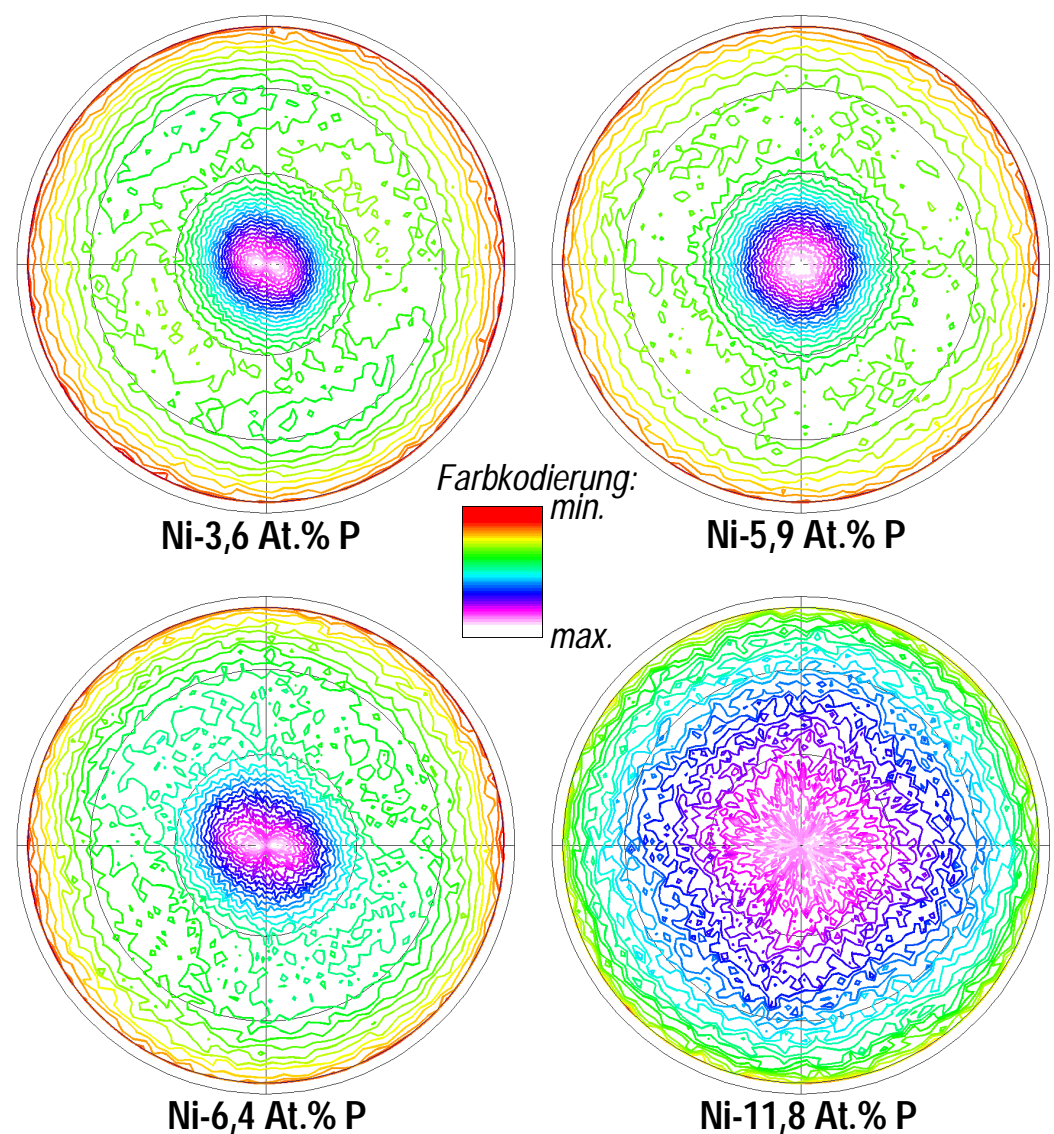

Abb. 4.3 (111)-Polfiguren der Texturanalysen der Ni-P-Legierungen im Abscheidezustand. Alle Proben weisen deutliche (111)-Texturen in Richtung der Probennormalen auf.

Es ist die gemessene Intensiät der (111)-Reflexe in Abhängigkeit der Dreh- und Kippwinkel zum Röntgenstrahl gezeigt. Kippwinkel zwischen 0 und 90 Grad entsprechen konzentrischen Kreisen um den Mittelpunkt, auf die wiederum die Drehwinkel 
zwischen 0 und 360 Grad projiziert sind. Die Farbkodierung der Intensitäten ist, wie üblicherweise dargestellt, nicht linear, sondern sie verläuft zwischen Minimum und Maximum proportional mit der Wurzel der Intensität. Gut zu erkennen ist, dass alle Proben eine bevorzugte (111)-Orientierung in Richtung der Probennormalen aufweisen. Mit Ausnahme der Ni-11,8 At.\% P-Probe sind die Texturen ausgesprochen ausgeprägt.

\subsubsection{Chemische Zusammensetzung}

Die P-Verteilungen in den nanokristallinen Strukturen der Ni-P-Legierungen im Abscheidezustand wurden mit der Tomographischen Atomsonde (TAP) bestimmt. Es wurden die Proben mit 3,6 At.\%, 5,9 At.\% und 11,8 At.\% Phosphor untersucht. In Abb. 4.4 sind entsprechend drei Konzentrationsprofile aus TAP-Analysen abgebildet. Die lokalen P-Konzentrationen wurden hier jeweils ermittelt mit Hilfe eines Analysezylinders mit einem Durchmesser von $3 \mathrm{~nm}$ und einer Höhe von 0,4 nm. Es ist zu erkennen, dass die lokalen P-Konzentrationen über die Messtiefe hinweg um die jeweiligen Mittelwerte herum schwanken. Die Abweichungen liegen größtenteils innerhalb der $2 \sigma$-Grenzen. Einzelne Peaks ragen jedoch teilweise über das $2 \sigma$-Intervall hinaus.
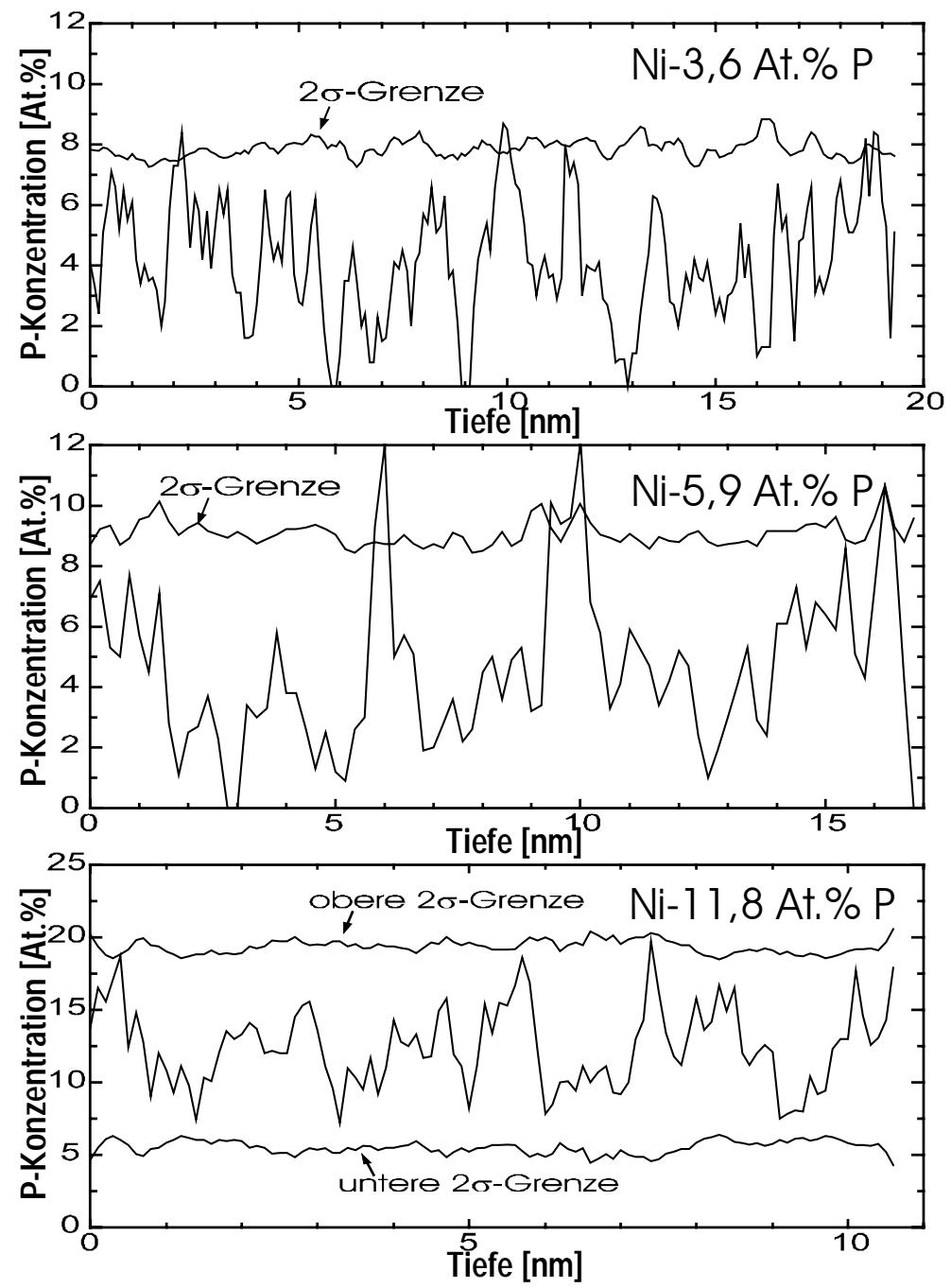

Abb. 4.4 Konzentrationsprofile aus TAP-Analysen von Ni-P-Legierungen im Abscheidezustand. 
Die TAP-Analysen wurden durchgeführt bei verhältnismäßig tiefen Proben-, respektive Kryostattemperaturen ( 25 bis $30 \mathrm{~K}$ ). Dadurch war gewährleistet, dass im Mittel ungefähr die nominelle P-Konzentration als mittlerer P-Gehalt bestimmt werden konnte, s. a. Kap. 2.4.4. Die Proben wurden dadurch allerdings auch sensibler gegenüber den mechanischen Belastungen während der Messungen, so dass sie i. d. R. nach 10 bis 20 nm Messtiefe abrissen. Längere Messungen konnten nur bei höheren Temperaturen $(50 \mathrm{~K})$ durchgeführt werden. Bedingt durch den in Kap. 2.4.4 beschriebenen Zusammenhang zwischen Kryostattemperatur und gemessener mittlerer Konzentration wurden dabei einerseits geringere mittlere P-Gehalte bestimmt als nominell zu erwarten. Andererseits konnte damit der Verlauf der P-Gehalte über größere Messtiefen hinweg beobachtet werden. In Abb. 4.5 ist das Konzentrationsprofil aus einer solchen längeren (160 nm Messtiefe) TAP-Messung einer Ni-5,9 At.\% P-Legierung abgebildet. Für die Bestimmung der lokalen P-Konzentration wurde hier, wie zuvor, ein Analysezylinder mit einem Durchmesser von $3 \mathrm{~nm}$ und einer Höhe von $0,4 \mathrm{~nm}$ verwendet. Deutlich zu erkennen ist, dass der Konzentrationsverlauf in seiner um den Mittelwert fluktuierenden Form über den gesamten Messtiefebereich gleich bleibt. Einzelne Peaks überragen wiederum das $2 \sigma$-Intervall.

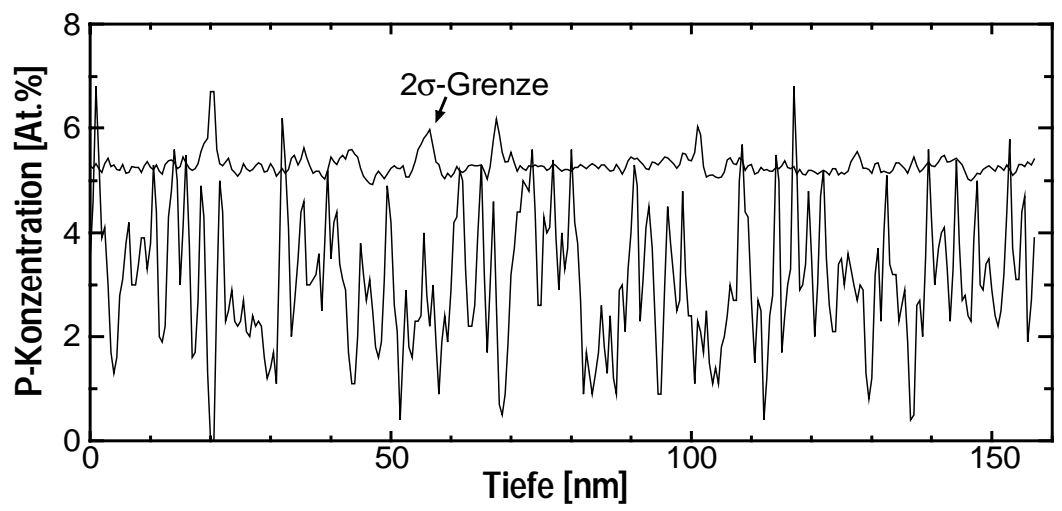

Abb. 4.5 Konzentrationsprofil aus einer längeren TAP-Analyse einer Ni-5,9 At.\% P-Legierung im Abscheidezustand. Die Kryostattemperatur während der Messung betrug $\mathrm{T}_{\mathrm{KK}}=50 \mathrm{~K}$, und war damit relativ hoch. Die mittlere P-Konzentration ist daher mit 3,1 At.\% geringer als der nominelle P-Gehalt (s. Kap. 2.4.4).

Aus den Konzentrationsprofilen heraus, ließ sich vermuten, dass die Ni-P-Legierungen keine homogenen P-Verteilungen aufwiesen. Um diese Hypothese zu überprüfen wurden $\chi^{2}$-Anpassungstests durchgeführt, wie sie in Kap. 2.4.3 beschrieben sind. Es wurden Stichproben á 50 bzw. 100 Atomen genommen und die Häufigkeitsverteilung des Phosphors in den Stichproben bestimmt. In Abb. 4.6 sind die Ergebnisse abgebildet. Im Falle der Proben mit 3,6 At.\% P und 5,9 At.\% P zeigen die gemessenen Verteilungen signifikante Abweichungen von einer korrespondierenden Binomialverteilung. Die gefundenen $\chi^{2}$-Werte (121 bzw. 206) liegen deutlich oberhalb der $\chi^{2}(\alpha=0,05)$ Vergleichswerte (16,9 bzw. 25). Die Legierungen sind also bereits signifikant inhomogen. Im Falle der Ni-11,8 At.\% P-Probe sind die Abweichungen der $\chi^{2}$-Wert nicht so groß $(26,0 \mathrm{zu} 21,0)$. Zwar überschreitet der $\chi^{2}$-Wert den Vergleichswert, eine eindeutige Schlussfolgerung bezüglich der Homogenität der P-Verteilung ist aber nicht möglich. 

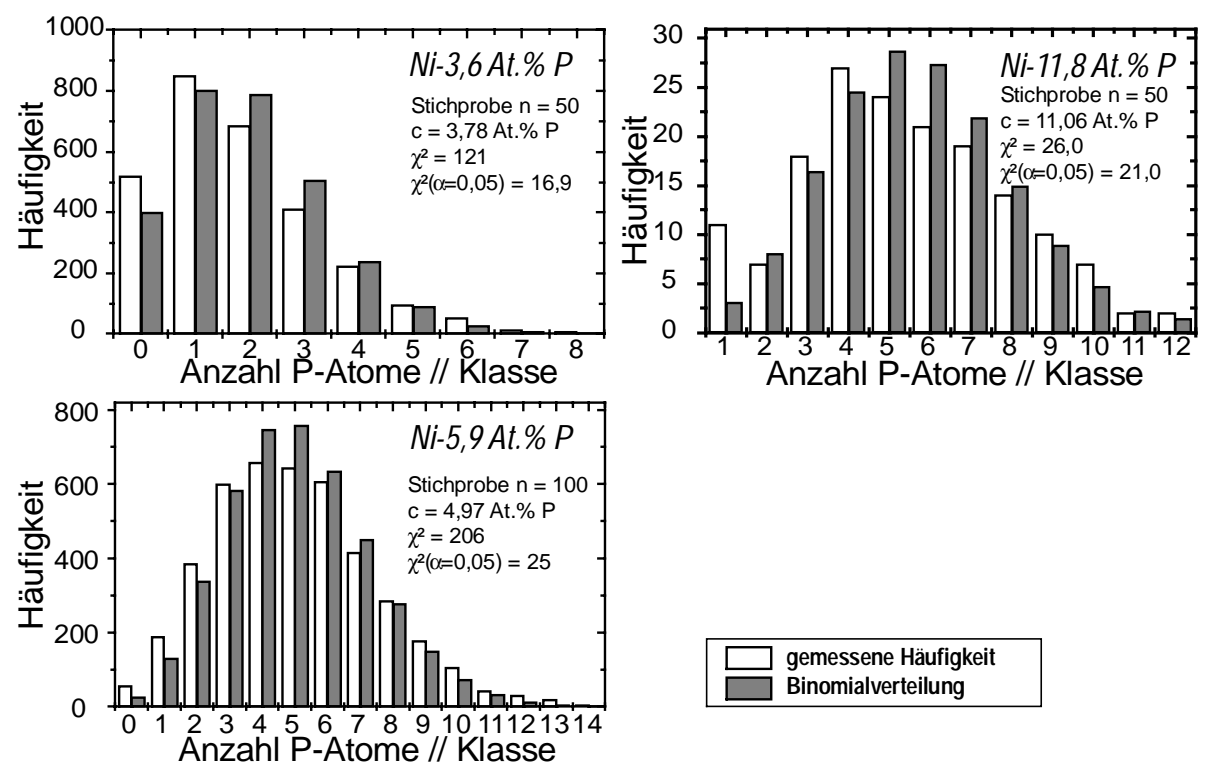

Abb. 4.6 Ergebnisse der $\chi^{2}$-Anpassungstests an Ni-P-Legierungen im Abscheidezustand. Dargestellt sind die gemessenen Häufigkeitsverteilungen des Phosphors in den Stichproben, sowie zum Vergleich die korrespondierenden Binomialverteilungen homogener Legierungen gleicher mittlerer Zusammensetzung. Die $\chi^{2}$-Werte und die dazugehörigen Vergleichswerte, $\chi^{2}(\alpha=0,05)$, sind in die Graphiken eingefügt.

\subsection{Entwicklung der Mikrostruktur durch Wärmebehandlung}

Die Ni-P-Legierungen wurden zunächst mit Hilfe der DSC-Anlage kalorimetrisch untersucht. Die Resultate sind im anschließenden Teilkapitel dargestellt. Anhand der Ergebnisse der kalorimetrischen Untersuchungen wurden dann gezielte Auslagerungen in derselben Anlage vorgenommen. Es wurden charakteristische Zustände vor und nach den in den Thermogrammen sichtbaren Umwandlungen präpariert. Diese Zustände wurden dann mittels XRD, FIM und TAP detailliert untersucht.

\subsubsection{Kalorimetrische Untersuchungen}

In isochron geführten Experimenten wurden von den Ni-P-Legierungen mit Hilfe einer DSC-Anlage Thermogramme erstellt, s. auch Kap. 2.5. Die Proben wurden mit einer Heizrate von $5{ }^{\circ} \mathrm{C}$ pro Minute bis auf $600{ }^{\circ} \mathrm{C}$ geheizt. Die Thermogramme zeigten alle einen ähnlichen charakteristischen Kurvenverlauf. Sie sind in Abb. 4.7 dargestellt. Bei ca. $120{ }^{\circ} \mathrm{C}$ setzt eine erste exotherme Reaktion ein, die sich über einen weiten Temperaturbereich bis ca. $300{ }^{\circ} \mathrm{C}$ erstreckt. Außerdem weisen die Thermogramme einen zweiten vergleichsweise scharfen exothermen Peak auf, der - abhängig von der Legierung - bei Temperaturen zwischen 350 bis $400{ }^{\circ} \mathrm{C}$ auftritt. Durch Auswertung der Kurven wurden die Reaktionswärmen der beiden Reaktionen sowie die StartTemperaturen für die zweite Reaktion bestimmt. In Tab. 4.3 sind die Ergebnisse zusammengefasst. Dabei ist anzumerken, dass die Unsicherheit bei der Bestimmung der Reaktionswärmen relativ groß ist. Bei den recht scharfen Peaks der 2. Umwandlung wird sie auf $20 \%$ des Messwertes geschätzt. Für die sehr breiten ersten Peaks muss eine mindestens doppelt so große Unsicherheit angenommen werden. Die Temperaturwerte für die ersten Reaktionen konnten aufgrund der flachen Kurvenverläufe lediglich auf ca. $120{ }^{\circ} \mathrm{C}$ abgeschätzt werden. Zusätzlich zu den beiden großen Peaks ist in den Thermogrammen auch noch ein kleiner Peak bei ca. $345{ }^{\circ} \mathrm{C}$ zu erkennen. Der Peak wurde bei den jeweils ersten Durchläufen nicht beobachtet. Er ist vermutlich auf die magnetische Entordnung der spontanen Magnetisierung der (ferromagnetischen) Proben beim 
zweiten Referenzdurchlauf zurückzuführen (zum Vergleich: die Curie-Temperatur reinen Nickels beträgt ca. $354{ }^{\circ} \mathrm{C}$ [KITT93]). Aufgrund der Differenzbildung der Referenzkurve mit der Kurve aus dem ersten Durchlauf erscheint der Peak exotherm, obwohl die zugrundeliegende Umwandlung endotherm ist. Dieser Peak wird nicht weiter berücksichtigt.

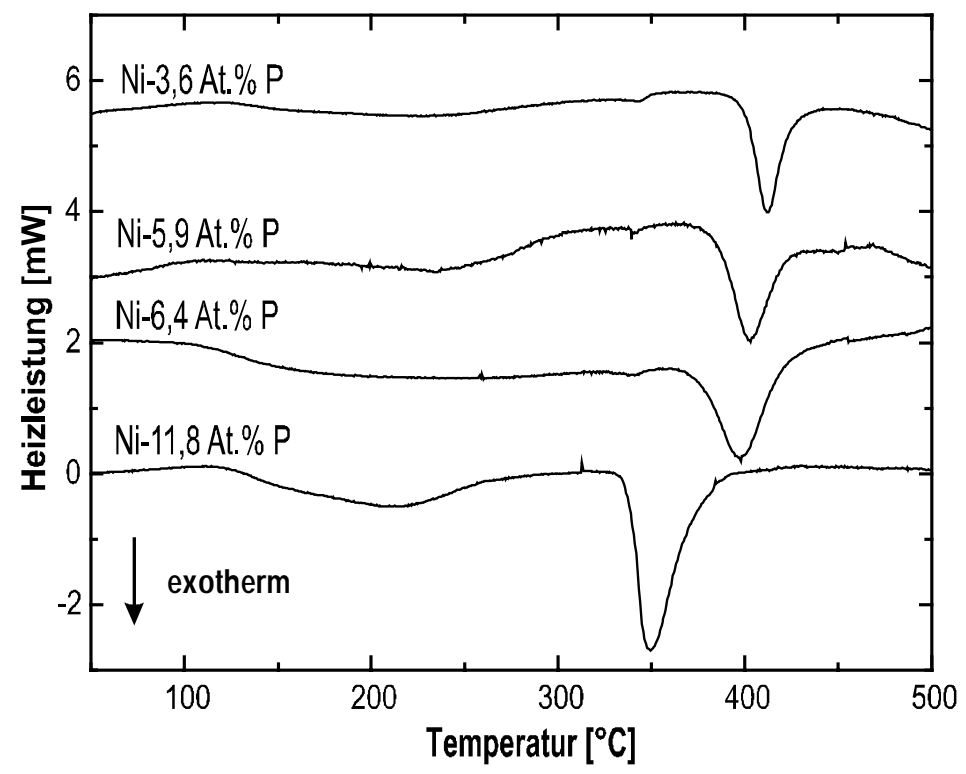

Abb. 4.7 Thermogramme der Ni-P-Legierungen. Die Proben wurden mit einer Heizrate von $5{ }^{\circ} \mathrm{C} / \mathrm{min}$ bis auf $600{ }^{\circ} \mathrm{C}$ geheizt.

\begin{tabular}{|c|c|c|c|c|}
\hline \multirow{2}{*}{$\begin{array}{c}\text { Probe / } \\
\text { P-Gehalt [At. \% }]\end{array}$} & \multicolumn{2}{|c|}{ Reaktion 1 } & \multicolumn{2}{c|}{ Reaktion 2 } \\
\cline { 2 - 5 } & $\mathbf{T}_{\mathbf{1}}\left[{ }^{\circ} \mathbf{C}\right]$ & $\Delta_{\mathbf{H}}[\mathbf{~ k J / m o l}]$ & $\mathbf{T}_{\mathbf{2}}\left[{ }^{\circ} \mathbf{C}\right]$ & $\boldsymbol{\Delta H}_{\mathbf{2}}[\mathbf{k J} / \mathbf{m o l}]$ \\
\hline 3,6 & ca. 120 & $-1,3$ & 400 & $-1,1$ \\
\hline 5,9 & ca. 120 & $-1,5$ & 390 & $-1,4$ \\
\hline 6,4 & ca. 120 & $-1,6$ & 381 & $-1,6$ \\
\hline 11,8 & ca. 120 & $-1,9$ & 338 & $-2,5$ \\
\hline
\end{tabular}

Tab. 4.3 Reaktionswärmen und Start-Temperaturen der exothermen Reaktionen in der thermischen Analyse der Ni-P-Legierungen.

\subsubsection{Entwicklung der Korngrößen und Phasenbildung}

Die Untersuchungen der Korngrößen und Phasenzusammensetzung im Abscheidezustand wurden bereits im Kap. 4.1.2 beschrieben. Darüber hinaus wurden die Ni-PProben in der DSC-Anlage ausgelagert. Um die Entwicklung der Korngrößen und die Bildung neuer Phasen zu untersuchen wurde die XRD eingesetzt. Mit Hilfe von FIMUntersuchungen wurde das gewonnene Bild vervollständigt. Die Auslagerungen erfolgten, wie bei der thermischen Analyse, isochron mit konstanten Heizraten von $5{ }^{\circ} \mathrm{C} / \mathrm{min}$. Die Proben wurden ausgelagert bis $\mathrm{zu}$ Temperaturen, die anhand der Thermogramme ausgewählt wurden. Dies waren die Zustände nach der ersten Umwandlung bei $320{ }^{\circ} \mathrm{C}$, kurz vor der zweiten Umwandlung nahe der jeweiligen StartTemperatur und nach der zweiten Umwandlung bei $450{ }^{\circ} \mathrm{C}$. Eine 6,4 At.\% P-Probe 
wurde auch bis zur zweiten Peaktemperatur $\left(397{ }^{\circ} \mathrm{C}\right)$ ausgelagert, um den Zustand während der zweiten Umwandlung untersuchen zu können. Außerdem wurden Proben für die Dauer einer Stunde bei $320^{\circ} \mathrm{C}$ ausgelagert.

Bei den isochron ausgelagerten Proben wurden in den Röntgendiffraktogrammen bis zur Start-Temperatur der zweiten Umwandlung bei allen Proben ausschließlich verbreiterte fcc-Nickel Reflexe beobachtet. Bei $450{ }^{\circ} \mathrm{C}$ waren dann zusätzliche Peaks zu finden, die einer $\mathrm{Ni}_{3} \mathrm{P}$-Phase zuzuordnen sind. Diese Reflexe traten auch bereits im Falle der Ni6,4 At.\% P-Probe auf, die bis wenig oberhalb der Start-Temperatur (bis $397{ }^{\circ} \mathrm{C}$ ) ausgelagert wurde. In Abb. 4.8 ist beispielhaft, die Sequenz von Diffraktogrammen einer Ni-5,9 At.\% P-Legierung dargestellt. Um auch Reflexe geringer Intensität zu visualisieren wurden die Intensitäten logarithmisch aufgetragen. Es ist zu erkennen, dass bei $450{ }^{\circ} \mathrm{C}$ deutlich sichtbar erste $\mathrm{Ni}_{3}$ P-Reflexe auftreten und die fcc-Nickel-Reflexe sichtbar schärfer sind als bei tieferen Auslagerungstemperaturen. Es bestätigt sich damit, dass der zweite Umwandlungspeak in den Thermogrammen der Bildung der $\mathrm{Ni}_{3} \mathrm{P}$-Phase entspricht. Zusätzlich nimmt offensichtlich die mittlere Korngröße zu.
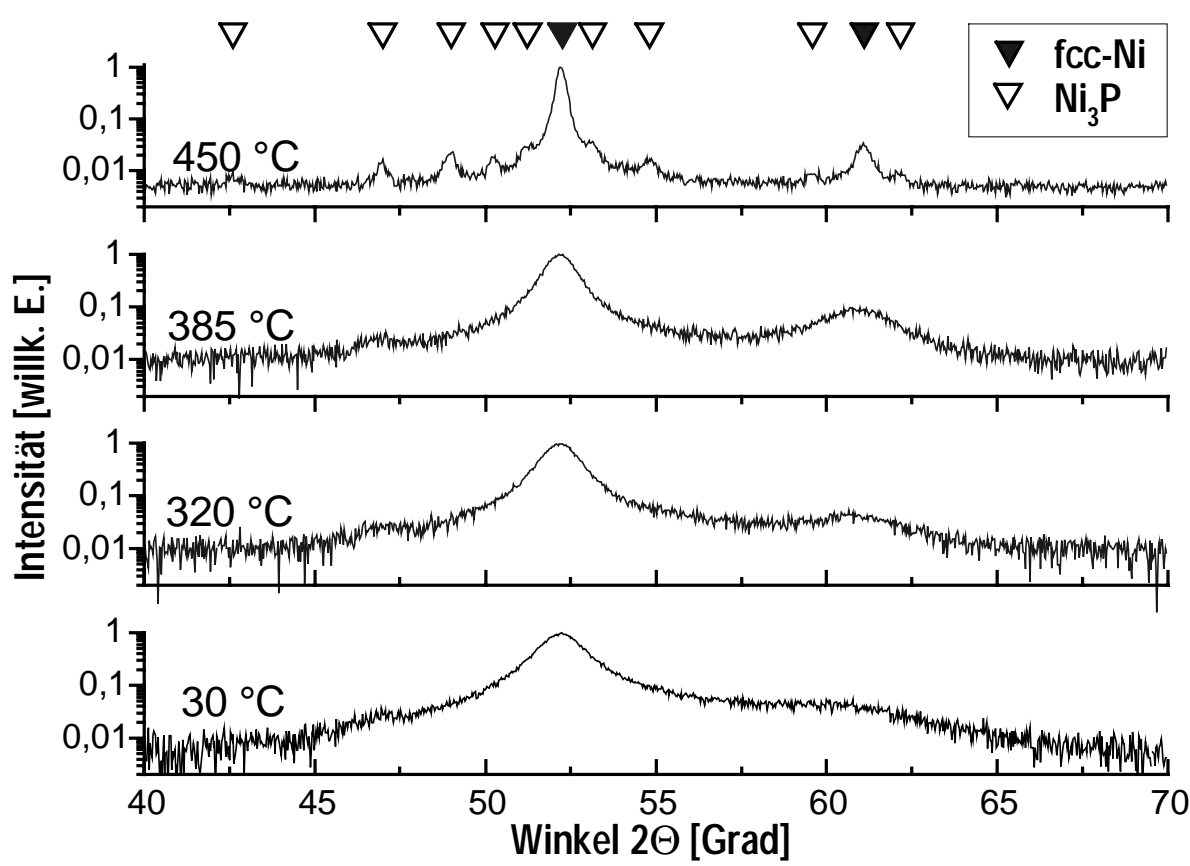

Abb. 4.8 Röntgendiffraktogramme einer Ni-5,9 At.\% P-Legierung nach verschiedenen isochronen Auslagerungen. Die Positionen der fcc-Ni(P)-Reflexe sind am oberen Bildrand mit ausgefüllten Dreiecken markiert, die der $\mathrm{Ni}_{3} \mathrm{P}$-Reflexe mit offenen Dreiecken.

Mittels der Scherrer-Formel, Gl. 2.6, konnten die mittleren Korndurchmesser der jeweiligen Zustände aus den Röntgendiffraktogrammen genauer bestimmt werden. Sie sind in Tab. 4.4 zusammengefasst. Zur Veranschaulichung der Entwicklung der Korngrößen sind die Daten des Abscheidezustandes und der isochronen Auslagerungen auch in Abb. 4.9 noch einmal in graphischer Form visualisiert. Es wird deutlich, dass die Korngrößen nahezu stabil bleiben und nur geringfügig zunehmen bis die $\mathrm{Ni}_{3} \mathrm{P}-$ Bildung einsetzt. Danach vergröbern sich die Strukturen erheblich, die Korngrößen nehmen zu um das drei- bis fünffache. 


\begin{tabular}{|c|c|c|c|c|c|c|c|c|}
\hline \multirow{2}{*}{$\begin{array}{c}\text { Auslagerung } \\
\text { isochron: } \\
5^{\circ} \mathrm{C} / \mathrm{min} \\
\end{array}$} & \multicolumn{2}{|c|}{ Ni-3,6 At. \% P } & \multicolumn{2}{|c|}{ Ni-5,9 At.\% $P$} & \multicolumn{2}{|c|}{ Ni-6,4 At. \% P } & \multicolumn{2}{|c|}{$\mathrm{Ni}-11,8$ At. $\% \mathrm{P}$} \\
\hline & D [nm] & $\mathbf{N i}_{3} \mathbf{P}$ & D [nm] & $\mathbf{N i}_{3} \mathbf{P}$ & $D$ [nm] & $\mathbf{N i}_{3} \mathbf{P}$ & $D[\mathbf{n m}]$ & $\mathbf{N i}_{3} \mathbf{P}$ \\
\hline $320^{\circ} \mathrm{C}$ & 9,1 & nein & 7,5 & nein & 7,2 & nein & 3,2 & nein \\
\hline $\begin{array}{l}\text { Start-Temper. } \\
\text { 2. Umwdlg. }\end{array}$ & $\begin{array}{c}12,2 \\
\left(400^{\circ} \mathrm{C}\right) \\
\end{array}$ & nein & $\begin{array}{c}9,2 \\
\left(385^{\circ} \mathrm{C}\right) \\
\end{array}$ & nein & $\begin{array}{c}8,4 \\
\left(375^{\circ} \mathrm{C}\right) \\
\end{array}$ & nein & $\begin{array}{c}3,9 \\
\left(337^{\circ} \mathrm{C}\right) \\
\end{array}$ & nein \\
\hline $\begin{array}{l}\text { Peak-Temper. } \\
\text { 2. Umwdlg. }\end{array}$ & - & - & - & - & $\begin{array}{c}13,5 \\
397^{\circ} \mathrm{C} \\
\end{array}$ & ja & - & - \\
\hline $450^{\circ} \mathrm{C}$ & 37,3 & ja & 32,7 & ja & 29,9 & $\mathrm{ja}$ & 23,4 & ja \\
\hline $\begin{array}{l}\text { isotherm: } \\
320{ }^{\circ} \mathrm{C}, 1 \mathrm{~h}\end{array}$ & 11,4 & nein & 8,4 & nein & 6,5 & nein & 11,7 & ja \\
\hline
\end{tabular}

Tab. 4.4 Korngrößen der Ni-P-Legierungen nach Auslagerung bei unterschiedlichen Temperaturen.

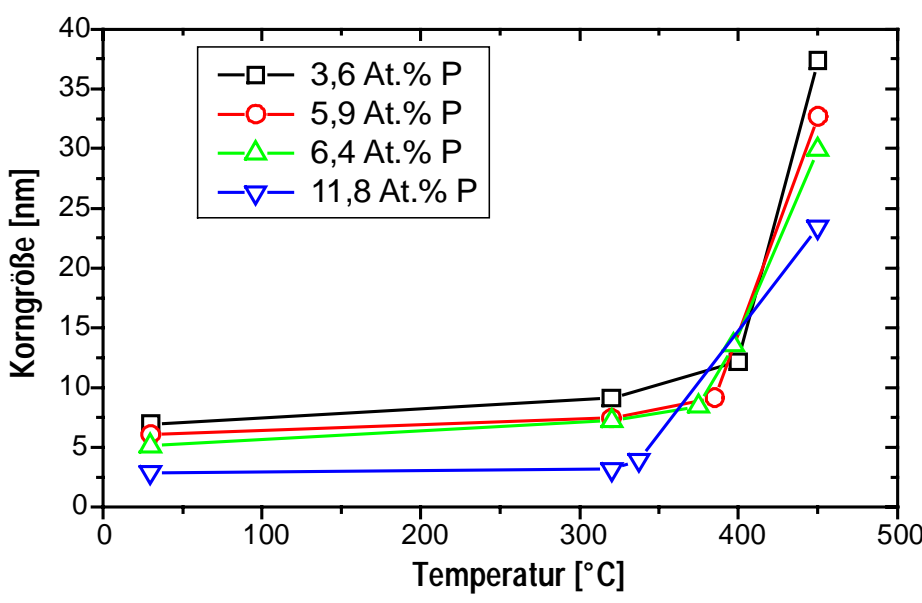

Abb. 4.9 Entwicklung der Korngrößen der Ni-P-Legierungen bei isochroner Auslagerung. Die Heizrate betrug $5{ }^{\circ} \mathrm{C} / \mathrm{min}$.

Die thermische Stabilität der Kornstruktur wird auch durch die Ergebnisse der isothermen Auslagerungen bei $320^{\circ} \mathrm{C}$ für $1 \mathrm{~h}$ bestätigt, die auch in Tab. 4.4 aufgeführt sind. Die Korngrößen nehmen nicht oder nur 1 bis $2 \mathrm{~nm}$ zu im Vergleich zu der isochronen Auslagerung bis $320{ }^{\circ} \mathrm{C}$. Eine Ausnahme bildet hier die Ni-11,8 At.\% P-Probe, bei der bereits die Keimbildung von $\mathrm{Ni}_{3} \mathrm{P}$-Ausscheidungen eingesetzt hat. In den - hier nicht abgebildeten - Diffraktogrammen konnten sehr deutlich größere $\mathrm{Ni}_{3} \mathrm{P}$-Reflexe beobachtet werden.

Aus den XRD-Untersuchungen konnten, wie oben gezeigt, die mittleren Korngrößen ermittelt werden. Korrespondierend $\mathrm{zu}$ diesen Ergebnissen zeigten auch FIMUntersuchungen Kontraste auf den gleichen Längenskalen. Sichtbar ausgeprägt waren die Kontraste jedoch nur bei ausgelagerten Zuständen. Die besten Abbildungen lieferten Spitzen unmittelbar nach Atomsonden-Messungen - sofern sie nicht abgerissen waren. Die Entwicklung durch Hochspannungs-Pulsen schien in diesem Fall vorteilhaft zu sein. In Abb. 4.10 sind FIM-Aufnahmen von drei Zuständen - (A) 3,6 At. \% P, $320^{\circ} \mathrm{C}, 1 \mathrm{~h}$, (B) 5,9 At. $\%$ P, $385{ }^{\circ} \mathrm{C}$, (C) 11,8 At. $\%$ P, $320{ }^{\circ} \mathrm{C}$ - abgebildet. In Bild (A) sind hell abbildende, ca. 10-15 nm große Bereiche - die Körner - mit den sie umgebenden dunklen Säumen zu erkennen. In Bild (B) ist eine ähnliche Struktur wie bei (A) zu erkennen auf einer Längenskala von 5-10 nm. Die Kontraste sind hier aber geringer 
ausgeprägt. In Bild (C) können Strukturen wie in (A) und (B) nicht mehr oder nur sehr vage aufgelöst werden. Es kann vermutet werden, dass es sich hierbei um einen chemischen Kontrast handelt. D. h., dass helle und dunkle Bereiche unterschiedliche chemische Zusammensetzungen aufweisen.

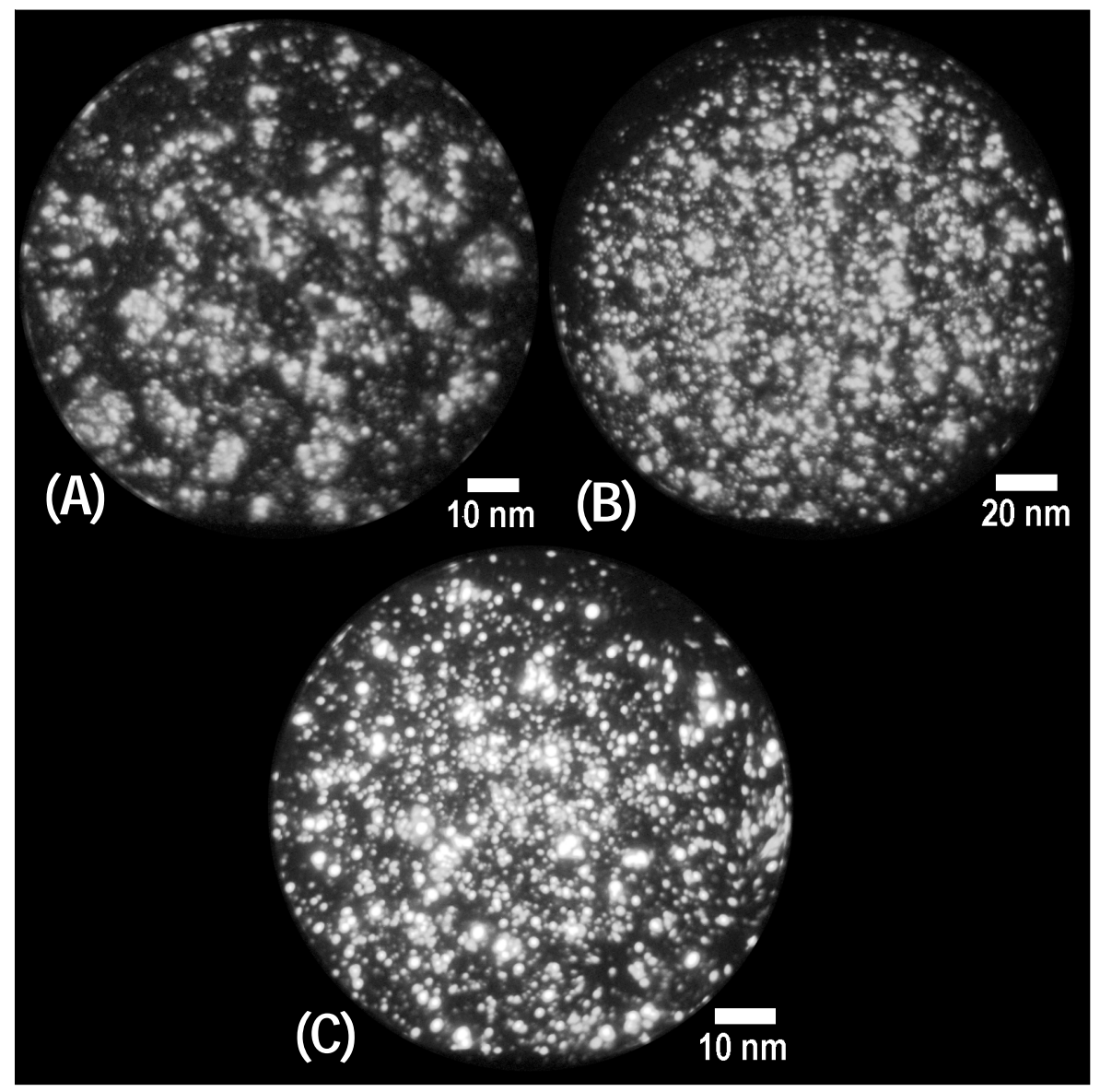

Abb. 4.10 FIM-Aufnahmen einzelner ausgelagerter Zustände von Ni-P-Legierungen: (A) 3,6 At.\% P, $320^{\circ} \mathrm{C}, 1 \mathrm{~h}$, (B) 5,9 At.\% P, $385{ }^{\circ} \mathrm{C}$, (C) 11,8 At.\% P, $320^{\circ} \mathrm{C}$. Hell abbildende Körner sind von dunklen Säumen umgeben. Der Kontrast deutet auf unterschiedliche chemische Zusammensetzung der Bereiche hin.

\subsubsection{Nanostrukturelle Veränderungen}

Die in den vorhergehenden Kapiteln dargestellten XRD-Untersuchungen hatten bereits gezeigt, dass die zweite Umwandlung, die in den Thermogrammen beobachtet wurde, auf die Bildung der $\mathrm{Ni}_{3} \mathrm{P}$-Phase zurückzuführen ist. Bis hin zu diesem Zustand zeigten die Proben eine ausgesprochene thermische Stabilität der nanokristallinen Struktur. Von besonderem Interesse war es daher, den Zustand nach der ersten Umwandlung genauer $\mathrm{zu}$ untersuchen. Es wurden TAP-Untersuchungen durchgeführt an ausgelagerten Ni-PLegierungen mit 3,6 At.\%, 5,9 At.\% und 11,8 At.\% P-Gehalt, um die PhosphorVerteilung in den nanokristallinen Strukuren zu analysieren. Die Ergebnisse der Untersuchungen bei den verschiedenen P-Konzentrationen sind in den folgenden Teilkapiteln im einzelnen dargelegt.

Außerdem wurden bei den TAP-Untersuchungen die analysierten Probenvolumina auch hinsichtlich der Existenz von in der 3D-Rekonstruktion nachweisbaren Gitterebenen einzelner Körner untersucht. Es wurden Teilvolumina selektiert und durch Drehen und 
Kippen ,per Auge“ begutachtet und es wurden 3D-Fourier-Analysen durchgeführt, um Periodizitäten im Realraum aufzuspüren. In keinem Fall konnten Gitterebenen in einer 3D-Rekonstruktion beobachtet werden. Sofern Ebenen hätten aufgelöst werden können, hätten diese eventuell mit den beobachteten P-Verteilung korreliert werden können. Die Grenzen der Auflösbarkeit waren bei den Ni-P-Legierungen aber offensichtlich überschritten.

\subsubsection{Untersuchungen an Nickel-3,6 At.\% Phosphor}

Zur Charakterisierung der P-Verteilung nach Wärmebehandlung in dieser Legierung sollen hier die TAP-Untersuchungen an zwei Zuständen dienen: (1.) isotherm ausgelagert bei $320{ }^{\circ} \mathrm{C}$ für $1 \mathrm{~h}$ und (2.) isochron ausgelagert bis $400{ }^{\circ} \mathrm{C}$ bei einer Heizrate von $5{ }^{\circ} \mathrm{C} / \mathrm{min}$.

Die ausgelagerten Proben mit 3,6 At.\% P erwiesen sich als verhältnismäßig stabil für TAP-Analysen. Bei dem Auslagerungszustand $320^{\circ} \mathrm{C}, 1 \mathrm{~h}$, gelang so eine Analyse mit einer Messtiefe von über $180 \mathrm{~nm}$. In Abb. 4.11 ist ein Konzentrationsprofil durch das Probenvolumen dargestellt. Das Test-Analysevolumen war in diesem Fall ein Zylinder mit 3,6 nm Durchmesser und 0,4 nm Höhe. Die mittlere Konzentration in dieser Messung wurde mit 3,51 At.\% P bestimmt und entsprach somit (nahezu) der nominellen Konzentration. Auffällig ist hier, dass eine Reihe von Peaks auftreten, die jeweils signifikante, die $2 \sigma$-Grenze deutlich überschreitende P-Anreicherungen repräsentieren. In der Graphik sind die Positionen dieser Peaks mit ausgefüllten Dreiecken gekennzeichnet. Die mittlere P-Konzentration an diesen Stellen beträgt ca. 10-11 At.\%. Auf einer Länge von $180 \mathrm{~nm}$ können 16 signifikante Peaks gezählt werden. Ihr mittlerer Abstand beträgt somit ca. $11 \mathrm{~nm}$.

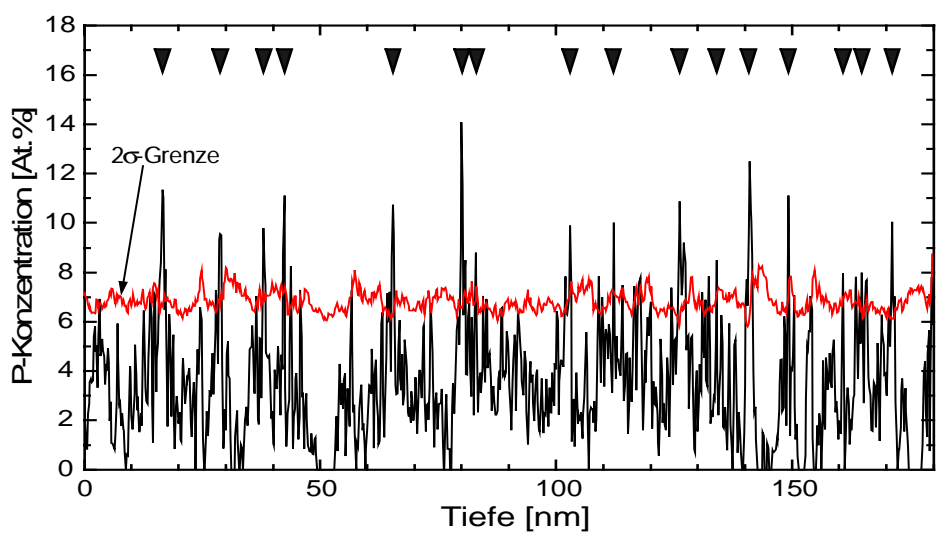

Abb. 4.11 Konzentrationsprofil aus der TAP-Analyse einer Ni-3,6 At.\% P-Legierung, ausgelagert bei $320{ }^{\circ} \mathrm{C}$ für $1 \mathrm{~h}$. Die ausgefüllten Dreiecke markieren signifikante P-Anreicherungen.

Diese Abstandsgröße steht in guter Übereinstimmung zu der mittleren Korngröße der Proben. Es ist daher naheliegend, dass die Phosphor-Anreicherungen entlang der Korngrenzen lokalisiert sind. Eine weitergehende Untersuchung der Morphologie der Anreicherungsbereiche zeigte unmittelbar deren 3-dimensionale umhüllende Gestalt. Dazu wurden in diskreten Abständen $(0,4 \mathrm{~nm})$ Konzentrationen innerhalb des gesamten Probenvolumens bestimmt. An jedem dieser Punkte wurde als Test-Analysevolumen eine würfelförmige Box der Größe $1,6 \times 1,6 \times 1,6 \mathrm{~nm}^{3}$ definiert, um die lokale Konzentration an dieser Stelle zu bestimmen. Zur Visualisierung der Konzentrationen im 3D-Raum wurden dann 2D-Querschnitte angefertigt auf denen die lokalen Konzentrationen als Farbpixel kodiert dargestellt sind. 


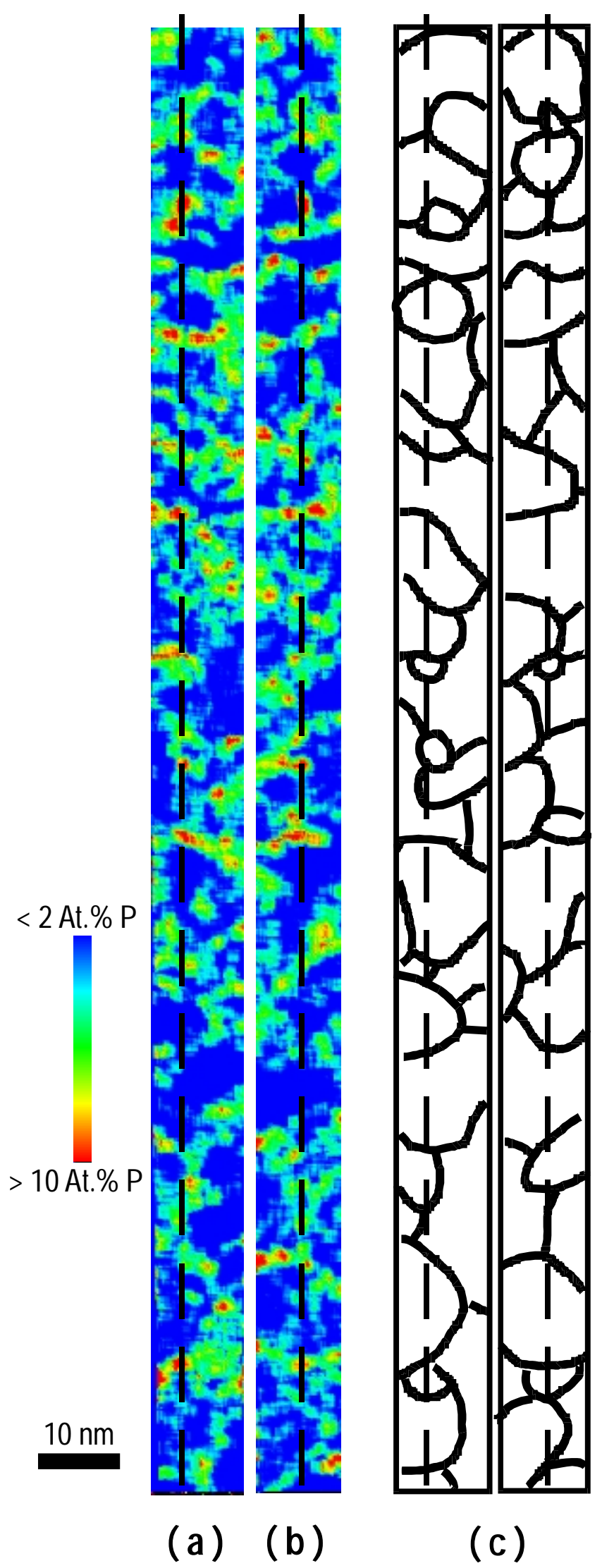

Abb. 4.12 Konzentrationsquerschnitte durch das Probevolumen einer mit TAP analysierten Ni-3,6 At.\% P-Legierung, die bei $320{ }^{\circ} \mathrm{C}$ für $1 \mathrm{~h}$ angelassen wurde. Auf der linken Seite sind zwei senkrecht zueinander stehende Querschnitte, deren gemeinsame Schnittlinie gestrichelt eingezeichnet ist, dargestellt. Auf der rechten Seite ist die Morphologie der mit Phosphor angereicherten Bereiche skizziert. Es ist naheliegend, dass diese mit der Korngrenzen-Morphologie identisch ist.

Diese Konzentrationsquerschnitte zeigen unmittelbar die Morphologie der mit Phosphor angereicherten Zonen. In Abb. 4.12 sind zwei solche, $11 \times 180 \mathrm{~nm}^{2}$ große Konzen- 
trationsquerschnitte dargestellt. Blaue Punkte repräsentieren niedrige Konzentrationen (geringer als 2 At.\% P) und rote hohe (größer als 10 At.\% P). Entsprechend der abgebildeten Farbskala sind dazwischenliegende Konzentrationen kodiert. Auf der rechten Seite der Abbildung wurde versucht, die Anordnung der Bereiche erhöhter PKonzentration nachzuzeichnen. Die skizzierte Morphologie entspricht offensichtlich der einer Korngrenzen-Morphologie. Die Dreidimensionalität der P-Verteilungen wird dadurch erkennbar, dass die beiden gezeigten Konzentrationsquerschnitte senkrecht zueinander stehen. Ihre gemeinsame Schnittlinie ist sowohl in den Querschnitten als auch in den korrespondierenden Skizzen als gestrichelte Linie eingezeichnet. Deutlich $\mathrm{zu}$ erkennen ist, dass die von erhöhten P-Konzentrationen umhüllten Bereiche sich in der dritten Dimension fortsetzen.

Zusammenfassend kann für die bei $320{ }^{\circ} \mathrm{C} \backslash 1 \mathrm{~h}$ angelassene Ni-3,6 At.\% P-Legierung festgestellt werden, dass der Phosphor sich im Bereich der Korngrenzen der nanokristallinen Struktur gleichförmig verteilt angereichert hat. Die lokalen Konzentrationen in diesen Bereichen liegen bei ca. 10 bis 11 At.\% P.

Bei höheren Temperaturen von $400{ }^{\circ} \mathrm{C}$ - gemäß der DSC- und XRD-Untersuchungen also kurz vor Beginn der $\mathrm{Ni}_{3} \mathrm{P}$-Ausscheidung - konnte eine ähnliche Struktur beobachtet werden. Für die Visualisierung der P-Verteilung in einer isochron bis $\mathrm{zu} 400{ }^{\circ} \mathrm{C}$ ausgelagerten Ni-3,6 At.\% P-Legierungen ist in Abb. 4.13 Teil (A) die 3D-Rekonstruktion des Probenvolumens einer TAP-Analyse gezeigt. Lediglich die P-Atome sind dargestellt. Ni-Atome wurden hingegen ausgeblendet, um die P-Verteilung sichtbar zu machen. Das Messvolumen beinhaltet zwei Bereiche - offensichtlich Körner -, die von einer P-angereicherten flächenhaften Zone umhüllt sind. Die Körner sind nicht vollständig innerhalb des analysierten Probenvolumens situiert, so dass man durch die angeschnittenen Seitenflächen hindurchschauen kann. Die Dreidimensionalität der PVerteilung ist in Teil (B) der Abbildung veranschaulicht. Auch hier wurden die lokalen P-Konzentrationen innerhalb des gesamten Probenvolumens ermittelt, wie bereits weiter oben im Text erläutert. In dieses Volumen wurden dann blau/gelb gestaltete Isokonzentrationsflächen, d. h. Flächen gleicher Konzentration (hier 2,5 At.\% P), eingezeichnet. Die gelbe Seite zeigt dabei in die Richtung höherer Konzentrationen und die blaue entgegengesetzt.

Zusätzlich konnte bei dieser Messung eine außergewöhnlich hohe P-Anreicherung beobachtet werden. Diese ist durch eine rote Isokonzentrationsfläche veranschaulicht, die eine Konzentration von 15 At.\% P repräsentiert. Bei der implizierten Kornstruktur liegt die markierte hohe Anreicherung in dem Teil der Korngrenze, der einem Tripelpunkt entspräche.

Die genauen P-Konzentrationen in der auffälligen P-Anreicherung, sowie innerhalb der Körner und in den Korngrenzen lassen sich aus den gezeigten Darstellungen nicht ablesen. Erst mit Hilfe gezielter Konzentrationsprofile konnten diese bestimmt werden. In Abb. 4.14 Teil (A) ist der Konzentrationsverlauf entlang der Volumenlängsachse dargestellt. Als Test-Analysevolumen wurde hier ein Quader der Länge und Breite $2 \times 2 \mathrm{~nm}^{2}$ und einer Höhe von $0,4 \mathrm{~nm}$ verwendet. Deutlich $\mathrm{zu}$ erkennen sind die drei Korngrenzen mit P-Anreicherungen von im Mittel 14 At.\%. Zwischen diesen Peaks, im Korninneren, geht der P-Gehalt nahezu auf Null zurück. Nach außen, in Richtung Korngrenzen steigt er etwas an. Im Mittel beträgt der P-Gehalt innerhalb des Kornes etwa 1 At.\%. Im zweiten Teil (B) der Abb. 4.14 ist eine Konzentrationsanalyse durch die genannte auffällige P-Anreicherung gezeigt. Die P-Konzentration erreicht Werte von 25 
At.\%. Dies ist insofern interessant, da diese Konzentration der einer $\mathrm{Ni}_{3} \mathrm{P}-\mathrm{Phase}$ entspricht.

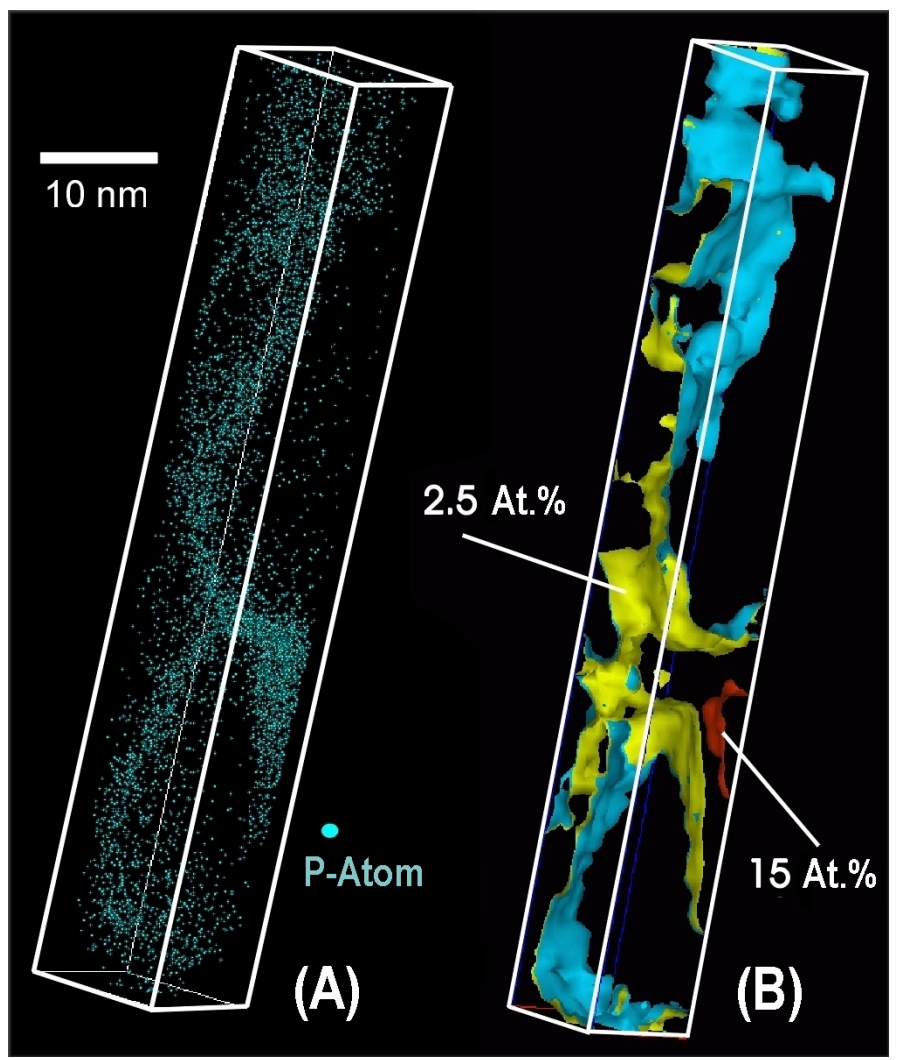

Abb. 4.13 (A) Rekonstruiertes, $10 \times 10 \times 60 \mathrm{~nm}^{3}$ großes Probenvolumen der TAP-Analyse einer Ni3,6 At.\% P-Legierung, die isochron bis $400{ }^{\circ} \mathrm{C}$ ausgelagert wurde. Jeder (hellblaue) Punkt repräsentiert ein $\mathrm{P}$ Atom. (B) Korrespondierende Isokonzentrationsflächen der P-Verteilung innerhalb desselben 3D-Volumens. Die blau/gelbe Fläche repräsentiert eine lokale Konzentration von 2,5 At.\% P. Die gelbe Seite zeigt in Richtung höherer, die blaue in Richtung geringerer Konzentrationen. Die rote Fläche repräsentiert 15 At. \% $\mathrm{P}$. Sie markiert eine auffällig hohe $\mathrm{P}-$ Anreicherung.
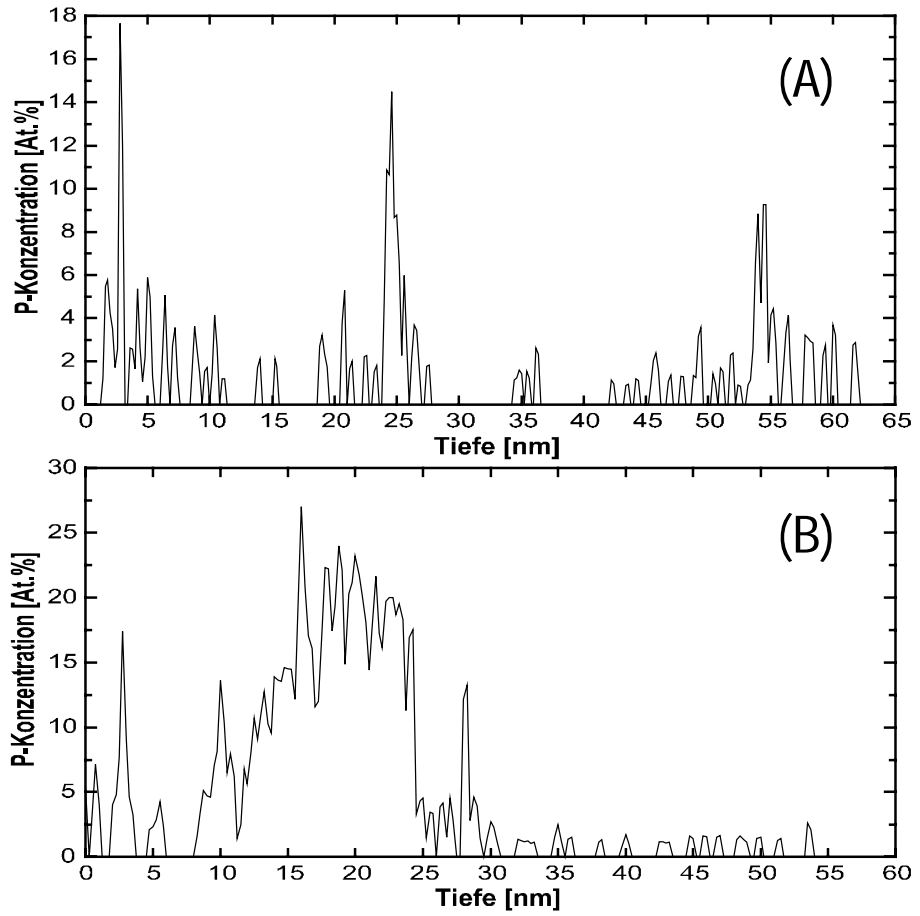

Abb. 4.14 Konzentrationsprofile aus der TAP-Analyse einer Ni3,6 At.\% P-Legierung, isochron ausgelagert bis $400{ }^{\circ} \mathrm{C}$ : (A) Konzentrationsverlauf durch zwei Körner und die umgebenden drei Korngrenzen mit P-Anreicherungen von ca. 14 At.\%. (B) Profil durch eine auffällige P-Anreicherung, s. Abb. 4.13. Der PGehalt beträgt bis zu 25 At.\%. 


\subsubsection{Untersuchungen an Nickel-5,9 At.\% Phosphor}

Die Entwicklung der P-Verteilung in den 5,9 At.\% P Proben bei Wärmebehandlung kann anhand von TAP-Untersuchungen an zwei Zuständen demonstriert werden: Ni5,9 At.\% P, isochron ausgelagert bis (1.) $320{ }^{\circ} \mathrm{C}$ und bis (2.) $385^{\circ} \mathrm{C}$ bei Heizraten von $5{ }^{\circ} \mathrm{C}$ pro Minute. Auch diese Proben verhielten sich in den TAP-Messungen relativ stabil, so dass bezogen auf die Größe der Kornstruktur ausreichend große Volumina analysiert werden konnten. Allerdings wurden bei den bis $320^{\circ} \mathrm{C}$ angelassenen Proben die Messungen bei Kühlkopftemperaturen von $40 \mathrm{~K}$ durchgeführt, so dass die gemessenen mittleren P-Gehalte mit ca. 4 bis 4,5 At.\% etwas niedriger bestimmt wurden als das nominell zu erwarten war, s. a. Kap. 2.4.4.

Die Ergebnisse von TAP-Analysen an einer bis $320 \mathrm{C}$ geheizten Ni-5,9 At.\% P-Legierung sind in Abb. 4.15 und Abb. 4.16 dargestellt. Erstere Abbildung zeigt ein Konzentrationsprofil durch das analysierte Volumen. Das Test-Analysevolumen hatte hier die Form eines Zylinders mit einem Durchmesser von $3 \mathrm{~nm}$ und einer Höhe von 0,4 nm. Über die Messtiefe von $45 \mathrm{~nm}$ hinweg sind 11 Peaks zu erkennen, die über den $2 \sigma$ Bereich - zum Teil nur geringfügig - hinausragen. Jedoch nur 5 bis 6 P-Anreicherungen übertreffen die Grenze deutlich, wenn man als Kriterium eine ein-prozentige Überhöhung annimmt. Ihre P-Konzentration liegt im Mittel bei ca. 11 At.\% und ihre mittlere Breite beträgt ca. $1 \mathrm{~nm}$. In der Abbildung sind ihre Positionen durch ausgefüllte Dreiecke markiert. Diese hier gefundenen P-Anreicherungen verteilten sich im Volumen ein wenig ähnlich, wie es bei den Proben mit 3,6 At.\% P im vorigen Kapitel dargestellt wurde. Es sind Merkmale zu erkennen, wie abgereicherte Zonen und linienartig angeordnete Anreicherungen, die eine Korngrenzen-Morphologie andeuten. In Abb. 4.16 sind zwei $20 \times 45 \mathrm{~nm}^{2}$ große Konzentrationsquerschnitte durch das analysierte Volumen dargestellt, die wiederum senkrecht zueinander stehen. Die gemeinsame Schnittlinie ist durch eine gestrichelte Linie kenntlich gemacht. Jeder Punkt repräsentiert eine lokale Konzentration innerhalb eines würfelförmigen Testvolumens von $(1,6 \mathrm{~nm})^{3}$. Blaue Bereiche zeigen eine P-Armut und rote Konzentrationen über 10 At. $\%$.

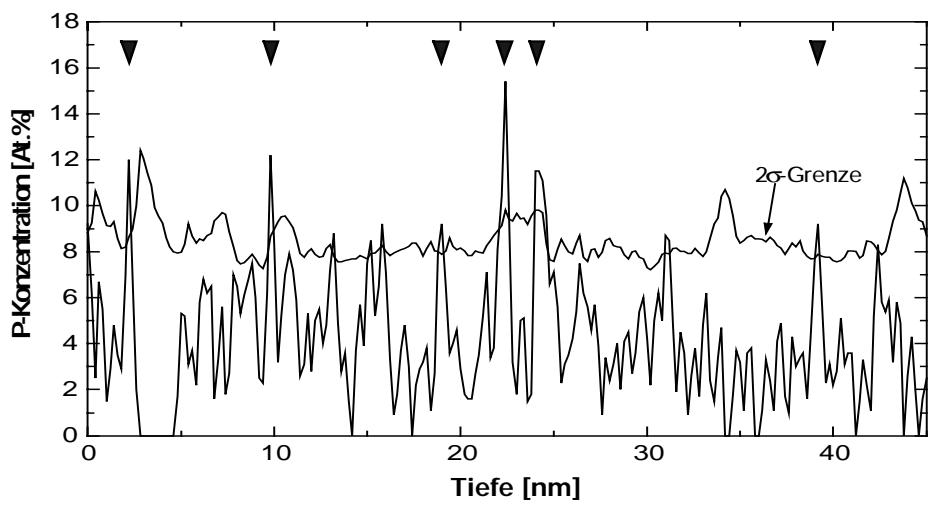

Abb. 4.15 Konzentrationsprofil aus der TAP-Analyse einer $\mathrm{Ni}$ 5,9 At.\% P-Legierung, die bis $320^{\circ} \mathrm{C}$ angelassen wurde. Die Positionen von deutlich die $2 \sigma-$ Grenze überragenden P-Anreicherungen sind mit schwarzen Dreiecken markiert. 

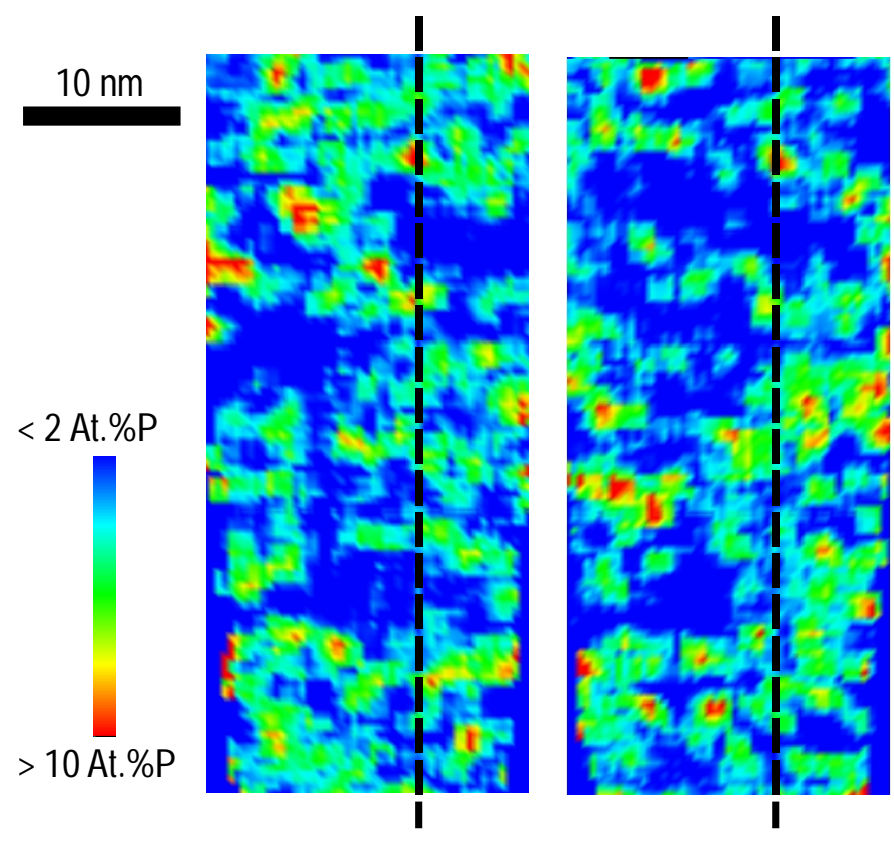

Abb. 4.16 Konzentrationsquerschnitte durch das Probenvolumen einer mit TAP analysierten $\mathrm{Ni}$ 5,9 At.\% P-Legierung, die isochron bis $320{ }^{\circ} \mathrm{C}$ ausgelagert wurde. Die gemeinsame Schnittlinie der senkrecht zueinander stehenden Querschnitte ist gestrichelt eingezeichnet. Eine Korngrenzen ähnliche Morphologie der P-Anreicherungen, wie sie Abb. 4.12 zu erkennen ist, kann bei diesem Auslagerungszustand nur erahnt werden.

Bei höheren Temperaturen, nach isochroner Auslagerung bis $385{ }^{\circ} \mathrm{C}$, also bis kurz unterhalb der kritischen Temperatur, bei der - gemäß der XRD-Untersuchungen - die $\mathrm{Ni}_{3} \mathrm{P}$-Phase sich ausscheidet, sind die Strukturen deutlich ausgeprägter. In Abb. 4.17 ist ein Konzentrationsprofil gezeigt, dass diesen Zustand repräsentiert. Das Test-Analysevolumen ist hier dasselbe wie im letzten Beispiel. Die P-Anreicherungen, deren Konzentrationen den $2 \sigma$-Bereich überschreiten, sind hier deutlich erkennbar. Sie haben einen mittleren Abstand von 8,5 nm und eine Breite von ca. $1 \mathrm{~nm}$. Die mittlere P-Konzentration dieser Peaks liegt etwas höher als im vorherigen Fall. Sie beträgt ca. 14 At.\% P. Zwischen den Peaks ist eine Abreicherung des Phosphors auf Werte von ca. 1 At. $\%$ zu beobachten.

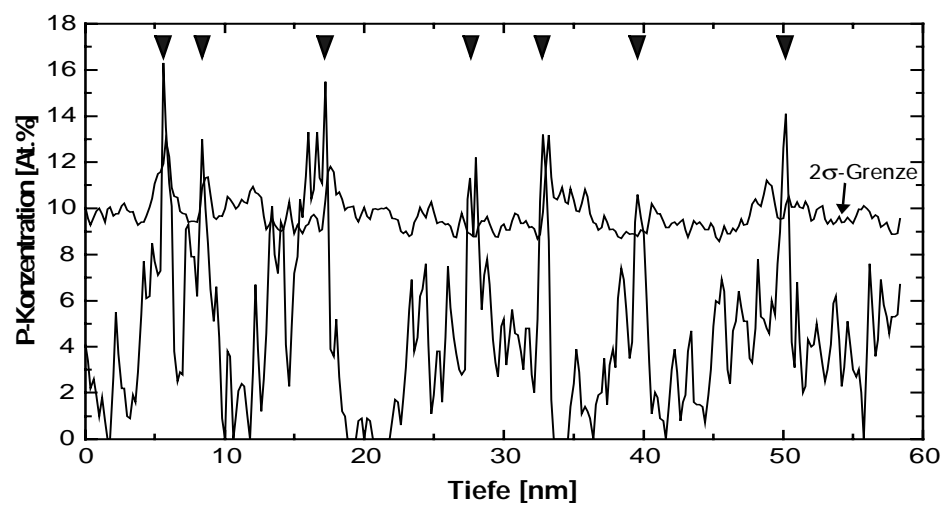

\begin{abstract}
Abb. 4.17 Konzentrationsprofil durch das Probenvolumen einer mit TAP analysierten, bis $385^{\circ} \mathrm{C}$ angelassenen Ni-5,9 At.\% P-Legierung. Sieben Peaks überragen deutlich die 2 $\sigma$-Grenze. Die PKonzentration zwischen den Peaks fällt auf Werte zwischen 0 und 2 At. $\%$ ab.
\end{abstract}

Die zu dieser Messung korrespondierenden Konzentrationsquerschnitte zeigen ein klares Bild der Morphologie der P-Anreicherungen. In Abb. 4.18 Teile (A) und (B) sind zwei zueinander senkrecht stehende Querschnitte abgebildet. Wiederum ist die gemeinsame Schnittlinie gestrichelt eingezeichnet. Das Test-Analysevolumen zur Bestimmung der lokalen Konzentrationen war hier, analog $\mathrm{zu}$ den vorherigen Beispielen, ein würfelförmiges Volumen der Größe $(1,6 \mathrm{~nm})^{3}$. Konzentrationen unterhalb von 3 At.\% sind durch die Farbe Blau repräsentiert. Größere lokale P-Gehalte 
von über 12 At.\% sind rot dargestellt. Dazwischenliegende Konzentrationen sind wiederum entsprechend der abgebildeten Farbskala zuzuordnen. Unschwer zu erkennen ist die Morphologie der mit Phosphor angereicherten Bereiche. Analog $\mathrm{zu}$ den Beobachtungen bei den Ni-3,6 At.\% P-Legierungen, die bei $320{ }^{\circ} \mathrm{C}$ für eine Stunde ausgelagert wurden, zeichnen auch hier die P-angereicherten Bereiche die charakteristische dreidimensionale umhüllende Morphologie der Korngrenzen eines nanokristallinen Gefüges nach. Die Dreidimensionalität des Gefüges wird anhand der senkrecht zueinander angeordneten Querschnitte sichtbar. In Teil (C) der Graphik sind die Verläufe der P-reichen Bereiche in den Querschnitten zur besseren Veranschaulichung der Struktur noch einmal als Linien skizziert. Der mittlere Durchmesser der implizierten Kornstruktur beträgt hier abgeschätzt ca. $10 \mathrm{~nm}$. Der Zusammenhang zwischen dem Konzentrationsprofil in Abb. 4.17 und den Konzentrationsquerschnitten wird anhand der gestrichelten Schnittlinie und der Dreicksmarkierungen am rechten Rand der Graphiken deutlich. Der Konzentrationsverlauf wurde entlang der Schnittlinie der beiden Querschnitte ermittelt. Die Markierungen am Rand korrespondieren unmittelbar mit denen in Abb. 4.17. Es kann unmittelbar nachvollzogen werden, dass die signifikanten Konzentrationspeaks an den Positionen der designierten Korngrenzen lokalisiert sind.

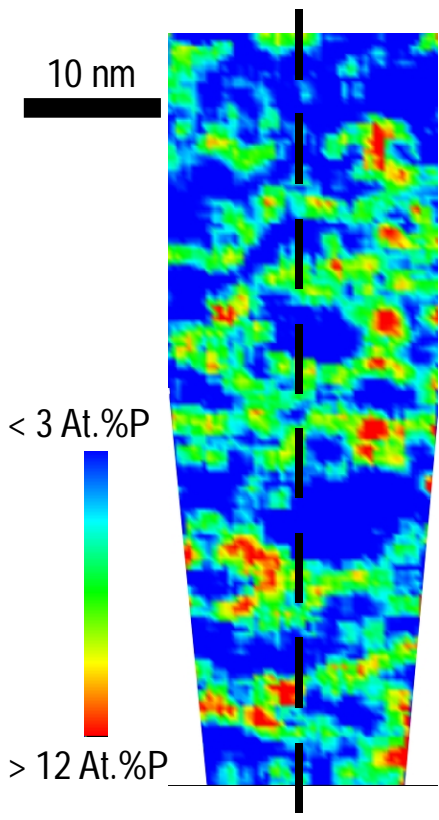

(A)

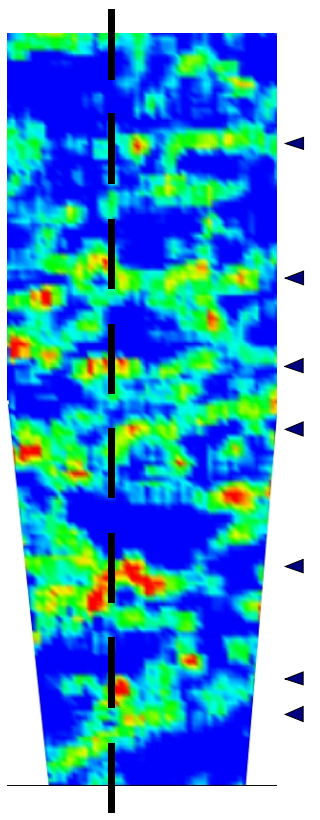

( B )

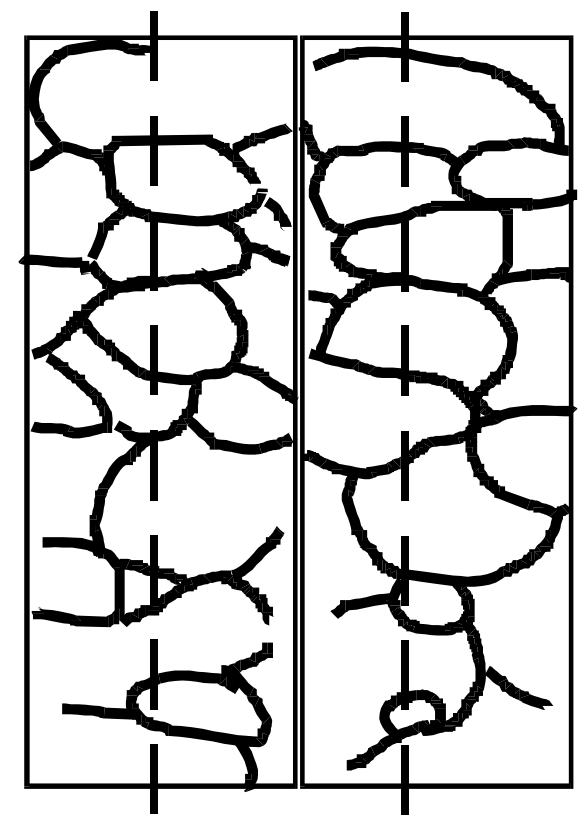

(C)

Abb. 4.18 Konzentrationsquerschnitte durch das Probenvolumen einer mittels TAP analysierten, isochron bis $385^{\circ} \mathrm{C}$ ausgelagerten Ni-5,9 At.\% P-Legierung. Teil (A) und (B) zeigen zwei senkrecht zueinander stehende Querschnitte, deren gemeinsame Schnittlinie gestrichelt eingezeichnet ist. Die Farbkodierung der lokalen Konzentrationen ist am linken Bildrand wiedergegeben. Die dreieckigen Markierungen am rechten Rand sind die korrespondierende Positionen von P-Anreicherungen des Konzentrationsprofiles aus Abb. 4.17. In Teil (C) ist die designierte Kornstruktur skizziert, entlang derer Phosphor angereichert ist.

\subsubsection{Untersuchungen an Nickel-11,8 At.\% Phosphor}

Die Untersuchungen an der Ni-11,8 At.\% P-Legierung gestalteten sich schwieriger als die zuvor beschriebenen. Auch im angelassenen Zustand waren die Proben in der TAP noch verhältnismäßig instabil, so dass die Messungen nach einigen Nanometern Tiefe 
durch Abriss der Spitzen terminiert waren. TAP-Analysen dieser Art wurden bei folgendem Auslagerungszustand durchgeführt: isochron geheizt bis $320{ }^{\circ} \mathrm{C}$ mit einer Heizrate von $5{ }^{\circ} \mathrm{C}$ pro Minute.

In Abb. 4.19 ist ein Konzentrationsprofil durch ein analysiertes Volumen dargestellt. Aufgrund der äußerst kleinen Kornstruktur der Legierung wurde, im Unterschied zu vorhergehenden Profilen, hier das Test-Analysevolumen schmaler gewählt. Es wurde ein Zylinder mit Durchmesser 1,4 nm und einer Höhe von $1 \mathrm{~nm}$ verwendet. Das Profil zeigt größere Konzentrationsschwankungen. An drei Stellen wird das $2 \sigma$-Intervall leicht über- bzw. unterschritten. Der erste sichtbare Peak zeigt eine lokale Konzentration von mehr als 25 At.\% P.

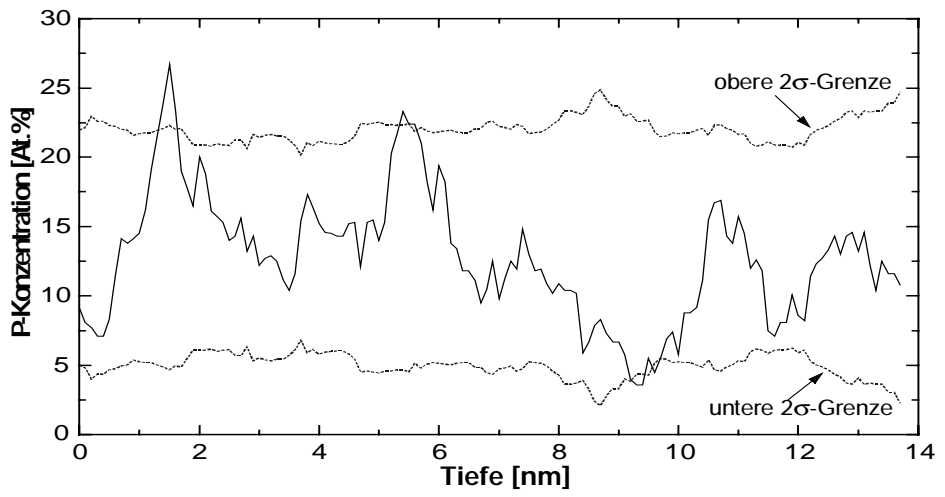

Abb. 4.19 Konzentrationsprofil aus einer TAP-Analyse einer isochron bis $320{ }^{\circ} \mathrm{C}$ getemperten Ni-11,8 At.\% P-Legierung.

Das Konzentrationsprofil deutet darauf hin, dass die P-Verteilung heterogen ist. Es werden bereits Konzentrationen über 20 At.\% beobachtet. Um zu kontrollieren, dass dies nicht nur auf die ohnehin hohe nominelle P-Konzentration mit den zu erwartenden relativ großen statistischen Schwankungen zurückzuführen ist wurde ein $\chi^{2}$ Anpassungstest, s. Kap. 2.4.3.1, durchgeführt. In Abb. 4.20 ist die Häufigkeitsverteilung von Phosphoratomen in Stichproben á 100 Atomen in einer TAP-Messung mit einem mittleren P-Gehalt von 11,7 At.\% dargestellt. Deutlich $\mathrm{zu}$ erkennen ist, dass die Verteilung stark von der korrespondierenden Binomialverteilung abweicht. Eine merkliche Anzahl an Stichproben weisst bereits mehr als 20 P-Atome, d. h. mehr als 20 At.\% P-Gehalt auf. Der $\chi^{2}$-Wert ist entsprechend sehr groß und dokumentiert die Abweichungen von der Binomialverteilung.

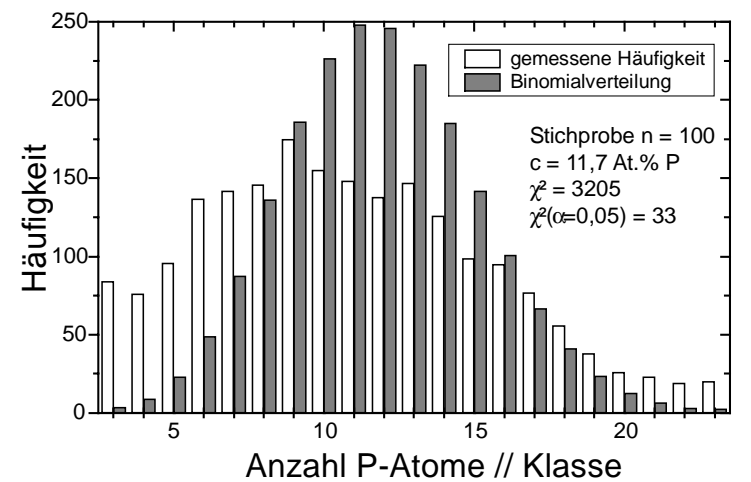

Abb. 4.20 Häufigkeitsverteilung des Phosphors in Stichproben á 100 Atomen in einer isochron bis $320{ }^{\circ} \mathrm{C}$ ausgelagerten Ni-11,8 At.\% P-Legierung. Zum Vergleich ist die korrespondierende Binomialverteilung einer (hypothetischen) homogenen Legierung eingezeichnet. Hohe Konzentrationen von 20 und mehr in den 100er Stichproben sind in merklicher Zahl vorhanden.

Ein Konzentrationsquerschnitt durch das analysierte Volumen zeigt Abb. 4.21. Das Test-Analysevolumen für die Ermittlung der lokalen Konzentration hatte eine Größe 
von $(1,4 \mathrm{~nm})^{3}$. Blau symbolisiert erneut eine niedrige P-Konzentration unterhalb 6 At.\% und Rot eine hohe von über 20 At.\%. Eine vergleichbar charakteristische Morphologie, wie sie in den vorangegangenen Untersuchungen bei niedrigeren P-Gehalten aufgezeigt wurde, ließ sich hier nicht erkennen. Zu erkennen ist aber, dass bereits eine Reihe von Bereichen existieren mit P-Anreicherungen von mehr als 20 At.\%.

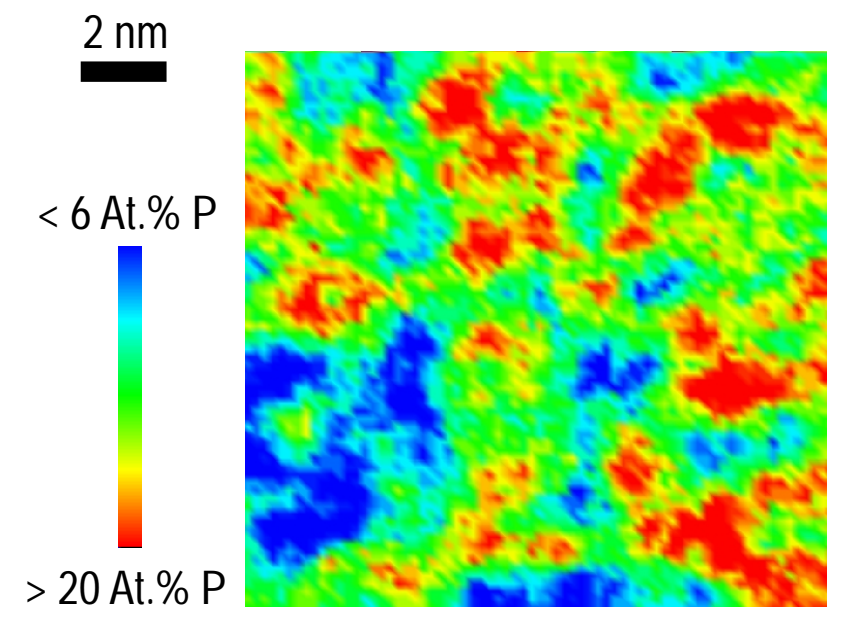

\begin{abstract}
Abb. 4.21 Konzentrationsquerschnitt durch das Probenvolumen einer mittels TAP analysierten, isochron bis $320^{\circ} \mathrm{C}$ ausgelagerten Ni-11,8 At.\% P-Legierung. Die Farbkodierung der Konzentration ist in der Farbskala auf der linken Seit wiedergegeben. Auffällig ist, dass mehrfach Konzentrationen über 20 At.\% P vorkommen.
\end{abstract}

\title{
4.2.4 Bestimmung des Korngrenzen-Exzess
}

Die in den vorangegangenen Kapiteln vorgestellten Untersuchungen haben gezeigt, dass Phosphor sich in den Korngrenzen anreichert, dort segregiert. Für die Beschreibung von Segregationsprozessen ist von besonderem Interesse die absolute Menge an Phosphor, die sich in oder in der Umgebung der Korngrenze anreichert. Die Anzahl der PhosphorAtome in der Grenzfläche bzw. der angereicherten Zone, $N^{P}{ }_{\text {seg }}$, bezogen auf die Größe der Korngrenzfläche, $A$, definiert den Korngrenzen-Exzess $\Gamma_{0}$. Es ist

$$
\Gamma_{0}=\frac{N_{s e g}^{P}}{A} .
$$

Die TAP-Untersuchungen ermöglichten es die Anzahl der segregierten P-Atome in den Korngrenzen unmittelbar auszuzählen und so den Korngrenzen-Exzess zu bestimmen. Es wurden die Zustände bei höheren Temperaturen und längeren Auslagerungszeiten gezielt untersucht, bei denen noch keine $\mathrm{Ni}_{3} \mathrm{P}$-Bildung zu beobachten war und bei denen die Segregation weit fortgeschritten und in ihrer Morphologie deutlich erkennbar war. Dies waren die beiden Legierungen mit 3,6 und 5,9 At.\% P bei den Auslagerungszuständen $320{ }^{\circ} \mathrm{C}$, $1 \mathrm{~h}$ (isotherm getempert) bzw. $385{ }^{\circ} \mathrm{C}$ (isochron geheizt mit $5{ }^{\circ} \mathrm{C} / \mathrm{min}$ ). Für die Untersuchungen wurden anhand der Konzentrationsquerschnitte gezielt geeignete Grenzflächen ausgewählt. Zylinderförmige Test-Analysevolumina, die den Bereich der Korngrenze überstreichen, wurden dann senkrecht zu den Grenzflächen orientiert. Die Zylinder hatten einen Radius von 2,0 nm. In Abb. 4.22 ist das Beispiel einer solchen Anordnung abgebildet. Dargestellt ist ein Ausschnitt aus einem Konzentrationsquerschnitt durch das analysierte Volumen einer getemperten Ni3,6 At.\% P-Legierung. In der Mitte des Bildes ist ein rechteckiger Bereich gestrichelt markiert, der die Position des zylinderförmigen Test-Analysevolumens wiedergibt. Auf halber Länge des Zylinders verläuft quer dazu die zu untersuchende mit Phosphor angereicherte Korngrenze. Die Lage des Zylinders senkrecht zur Bildebene wurde in 
gleicher Weise ausgerichtet anhand des senkrecht zum ersteren angeordneten Konzentrationsquerschnittes.

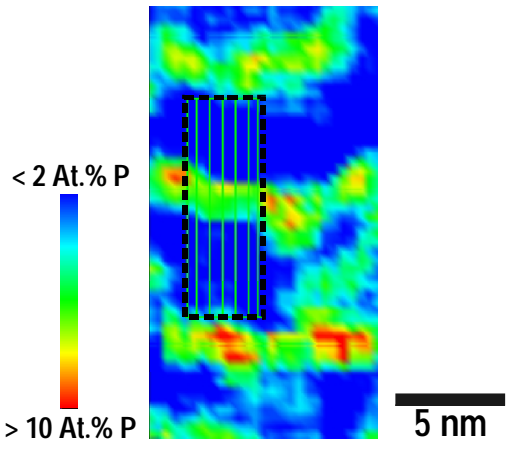

Abb. 4.22 Beispiel für die Anordnung des Test-Analysezylinders bei der Bestimmung des Korngrenzen-Exzesses. Dargestellt ist ein Konzentrationsquerschnitt durch das analysierte Volumen einer bei $320{ }^{\circ} \mathrm{C}$ ausgelagerten Ni-3,6 At.\% P-Legierung sowie, gestrichelt markiert, die Position des Zylinders.

Die Atome innerhalb des Test-Analysezylinders wurden dann bezüglich der Richtung der Zylinderachse in ihrer Reihenfolge des Erscheinens aufgelistet. Das Ergebnis wurde dann als Leiterdiagramm graphisch umgesetzt. Bei dieser Art der Darstellung wird ihrer Reihenfolge entsprechend - die kumulierte Anzahl der Phosphor-Atome als Funktion der kumulierten Anzahl der Nickel- und Phosphor-Atome dargestellt. Die lokale Steigung der resultierenden Kurve entspricht dann der lokalen Konzentration an dieser Stelle. In Abb. 4.23 sind beispielhaft für die beiden Legierungen zwei Leiterdiagramme dargestellt.
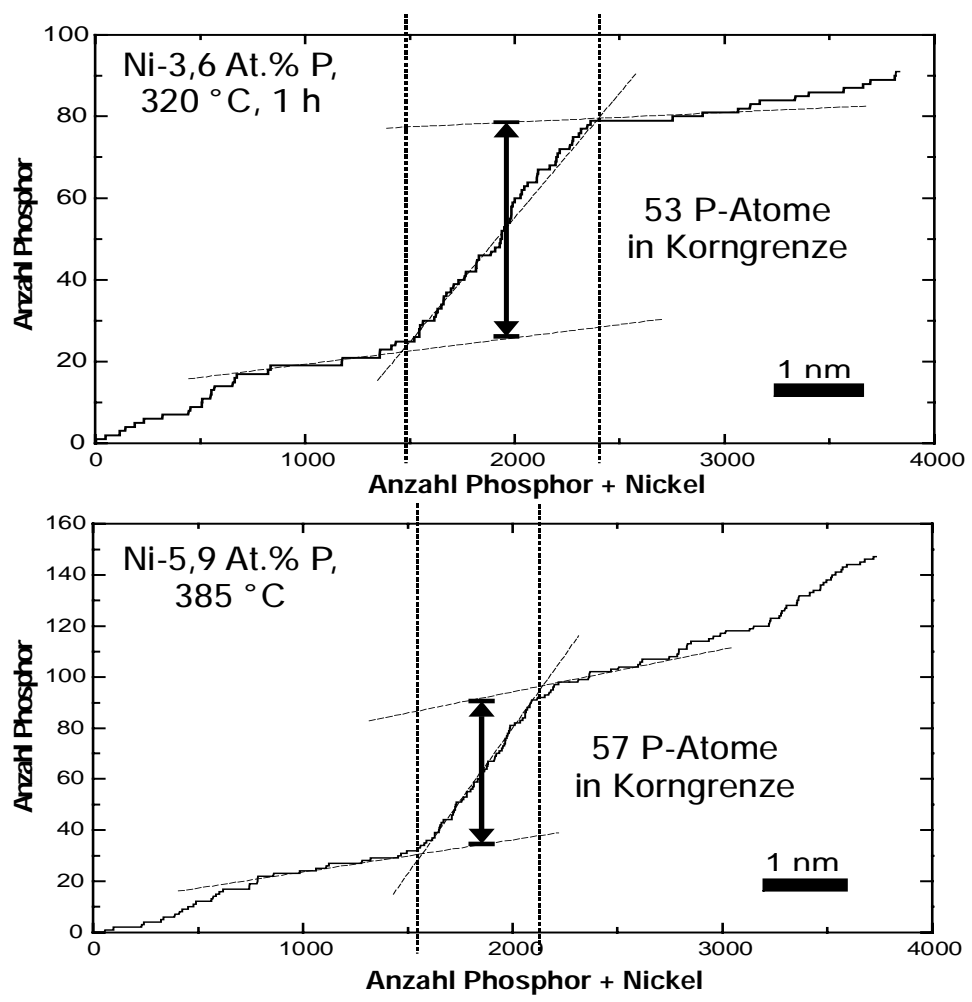

Abb. 4.23 Leiterdiagramme zur Ermittlung des KorngrenzenExzesses in getemperten Ni-PLegierungen mit 3,6 At.\% und 5,9 At.\% P. Die Grenzen der mit Phosphor angereicherten Zone sind durch gestrichelte senkrechte Linien markiert. Die Zahl der PAtome in der (Korngrenzen)-Zone entspricht der Länge des Doppelpfeils.

Es wurden dann in den Leiterdiagrammen die Konzentrationen links und rechts von, sowie in der Grenzfläche durch Einzeichnen der lokalen Tangenten graphisch ermittelt. Die Schnittpunkte definieren den Bereich der Segregationszone. In der Mitte dieser Zone liefert dann die Differenz zwischen der linken und rechten Tangente die Anzahl P- 
Atome, die sich zusätzlich in der Grenzfläche befinden. In Abb. 4.23 sind diese Größen als Doppelpfeile eingezeichnet. Daraus ließ sich unmittelbar der Korngrenzen-Exzess bestimmen. Die Fläche des Analysezylinders betrug $A=\pi \cdot(2 \mathrm{~nm})^{2}$. Zusätzlich musste außerdem noch die Detektoreffizienz bei den TAP-Analysen berücksichtigt werden. Sie betrug 0,5. Die reale Zahl von Atomen in der Grenzfläche ist also doppelt so groß anzusetzen wie die gemessene. In Tab. 4.5 sind die Ergebnisse von je vier Untersuchungen zusammengefasst. Es wurden mittlere Korngrenzen-Exzesse der PAtome von $9,2 \times 10^{18} \mathrm{~m}^{-2}$ und $8,5 \times 10^{18} \mathrm{~m}^{-2}$ für die Ni-3,6 At.\% P- bzw. Ni-5,9 At.\% PLegierung bestimmt. Im Rahmen der Schwankungsbreite der Messergebnisse sind die Exzess-Größen in beiden Legierungen offensichtlich gleich groß.

\begin{tabular}{|c|c|c|}
\hline Nr. & $\begin{array}{c}\text { Ni-3,6 At. \% P } \\
320^{\circ} \mathrm{C} \backslash 1 \mathrm{~h}\end{array}$ & $\begin{array}{c}\text { Ni-5,9 At.\% P } \\
385{ }^{\circ} \mathrm{C}\end{array}$ \\
\hline 1 & 42 & 50 \\
2 & 60 & 54 \\
3 & 75 & 53 \\
4 & 53 & 57 \\
\hline Mittelwert & 57,5 & 53,5 \\
\hline P-Exzess & $9,2 \times 10^{18}$ & $8,5 \times 10^{18}$ \\
$\Gamma_{0}[$ Atome/m & \\
\hline
\end{tabular}

Tab. 4.5 Ergebnisse aus der Ermittlung der Korngrenzen-Exzesse für getemperte Ni-3,6 At.\% P- und Ni-5,9 At.\% P-Legierungen. Gemessen wurde die Anzahl P-Atome in einer Korngrenzenfläche, die sich aus dem Querschnitt eines Test-Analysezylinders mit Radius $r=2 \mathrm{~nm}$ ergibt, s. Text. Bei der Berechnung des Exzesses wurde eine TAP-Detektoreffizienz von 0,5 berücksichtigt.

\subsection{Diskussion der Ergebnisse}

\subsubsection{Der Abscheidezustand}

Die in Kap. 4.1 dargelegten Untersuchungen zeigen, dass die chemisch abgeschiedenen Ni-P-Legierungen im Zustand nach der Herstellung nanokristalline Struktur aufweisen. Die mittels XRD bestimmten mittleren Korngrößen betragen zwischen 3 und $7 \mathrm{~nm}$, wobei mit zunehmendem P-Gehalt die Körner kleiner sind. Der gleiche Zusammenhang wird auch von anderen Autoren berichtet [ALLE82, KREY95]. Dabei ist dies sogar unabhängig von der Art der Herstellung. Auch die elektrolytisch abgeschiedenen Ni-PLegierungen verhalten sich ähnlich [VAFA78, BOYL91].

Alle untersuchten Ni-P-Schichten zeigen ferner deutliche bis starke kristallographische Texturen. Die (111)-Ebenen liegen bevorzugt parallel zur Schichtebene. Ihre Ebenennormalen zeigen in die Wachstumsrichtung der Schicht. Die Ausbildung dieser Art von Textur ist ein bekanntes Phänomen [GRAH65]. Auch bei elektrolytisch abgeschiedenem Nickel-Phosphor wird es beobachtet [MAED70], teilweise nur schwach ausgeprägt [OSMO92]. Die Textur erklärt auch, dass in den Diffraktogrammen die (200)-Reflexe stark bis vollständig unterdrückt sind. Und für die aus den Schichten präparierten TAPSpitzen bedeutet dies, dass es kaum Körner gibt, deren [111]-Richtung in etwa parallel zur Spiztenachse orientiert ist. Bei TAP-Analysen sind aber gerade die dichtest- 
gepackten Ebenen mit größten Abständen diejenigen, die in der 3D-Rekonstruktion noch am besten aufgelöst werden können. Dies könnte eine Ursache dafür sein, dass in den TAP-Analysen keine Ebenen in den 3D-Rekonstruktionen beobachtet wurden. Es ist allerdings auch möglich, dass allein durch den hohen Phosphor-Anteil in der Legierung die Auflösung reduziert ist. ${ }^{15}$

Als Phase wird mittels der XRD bei allen P-Gehalten im Abscheidezustand ausschließlich eine fcc-Nickel-Phase beobachtet. Die bei den hohen P-Gehalten im thermodynamischen Gleichgewicht vorliegende $\mathrm{Ni}_{3} \mathrm{P}$-Phase ist nicht vorhanden. Weder in den XRD-Untersuchungen gibt es Hinweise darauf, noch wurden in den TAP-Analysen entsprechend hohe P-Konzentrationen gefunden. Die Abwesenheit von $\mathrm{Ni}_{3} \mathrm{P}$ im Abscheidezustand wurde bisher auch von anderen Autoren mit XRD und TEM bestätigt. Sowohl in chemisch abgeschiedenen [KREY95, MÜLL96, ZHAN98], als auch in elektrolytisch abgeschiedenen Ni-P-Schichten [MAED70, VAFA78, MEHT95].

Über die Verteilung des Phosphors im Abscheidezustand ist bisher nur wenig bekannt. In fast allen Veröffentlichungen wird eine übersättigte Lösung des Phosphors in den Nickel-Körnern angenommen [MAED70, VAFA78, BOYL91, OSMO92, ZHAN98]. Allen und VanderSande sowie Mehta et al. [ALLE82, MEHT95] halten daneben auch eine Segregation des Phosphors in den Korngrenzen für möglich. Erstere vermuten, dass die Segregation des Phosphors bei der Herstellung der bestimmende Faktor für die resultierenden Korngrößen ist. Die Atomsonden-Analysen von Kreye et al. und Hentschel et al. [KREY95, HENT00] an einer chemisch abgeschiedenen Ni-3,6 At.\% PLegierung dokumentierten erstmals unmittelbar deren P-Verteilung im Abscheidezustand. Der Phosphor ist größtenteils in übersättigter Form in den Nickel-Körnern gelöst. Die Legierungen sind aber nicht homogen. Es werden einzelne P-Anreicherungen bis zu 10 At.\% beobachtet, die an den Korngrenzen vermutet werden. Die Ergebnisse in dieser Arbeit bestätigen dies. Darüberhinaus zeigen die TAP-Analysen, dass die Übersättigung der fcc-Ni-Phase mit Phosphor auch bei höheren Konzentrationen vorliegt. Die Proben mit 11,8 At.\% P sind dabei fast homogen, was die $\chi^{2}-$ Anpassungstests dokumentieren. Die Legierung mit 5,9 At.\% P ist auch stark übersättigt, weist aber zusätzlich signifikante Anreicherungen an Phosphor auf. Dass diese an den Korngrenzen lokalisiert sind, ist anzunehmen. Der Abscheidezustand ist also sowohl durch eine starke Überstättigung als auch eine schwache Korngrenzensegregation charakterisiert mit einer Kornstruktur, deren Größe mit zunehmendem P-Gehalt abnimmt. Dabei deutet alles darauf hin, dass diese Eigenschaften unabhängig von der Herstellungsmethode (chemische oder elektrolytische Abscheidung) sind. Der Ausgangszustand für folgende Wärmebehandlungen ist in etwa derselbe.

\subsubsection{Der Einfluss von Wärmebehandlungen}

Durch Wärmebehandlungen, d. h. Glühungen mit Temperaturen bis $\mathrm{zu} 320^{\circ} \mathrm{C}$ verändern sich die mittleren Korngrößen der Legierungen nur geringfügig und stabilisieren sich. ${ }^{16}$ Bei höheren, charakteristischen Temperaturen beginnt ein rasches Kornwachstum. Mit dieser Entwicklung verbunden sind deutliche Veränderungen in den Mikrostrukturen der Legierungen. Die in Kap. 4.2 vorgestellten Ergebnisse zeichnen ein klares Bild dieser Veränderungen. Zwei Prozesse dominieren dabei die Umwandlungen:

\footnotetext{
${ }^{15}$ Die Ursache liegt in den (geringfügigen) Unterschieden in der lokalen Feldverdampfung begründet, die je nach Phosphor-Anteil etwas variieren.

${ }^{16}$ Ausgenommen die 11,8 At.\%-ige Legierung bei der bereits nach $1 \mathrm{~h} \mathrm{Ni}{ }_{3} \mathrm{P}$-Bildung einsetzte.
} 
die Segregation des Phosphors in den Korngrenzen des nanokristallinen Gefüges und die Ausscheidung der $\mathrm{Ni}_{3} \mathrm{P}$-Phase. In den Thermogrammen werden entsprechend zwei exotherme Wärmetönungen gefunden.

Die zweite Umwandlung tritt bei (Start)-Temperaturen zwischen 337 und $400{ }^{\circ} \mathrm{C}$ auf ${ }^{17}$, wobei diese höher sind bei geringeren P-Gehalten. Die Umwandlung ist eindeutig der Ausscheidung der $\mathrm{Ni}_{3} \mathrm{P}-\mathrm{Phase}$ zuzuordnen. Die Röntgendiffraktogramme zeigen bis zu den Starttemperaturen keine $\mathrm{Ni}_{3} \mathrm{P}$-Reflexe. Erst bei der Peak-Temperatur bzw. oberhalb davon treten diese hervor. Die gefundenen Werte sind in guter Übereinstimmung mit Daten, die von anderen Autoren berichtet wurden [ALLE82, MEHT95, KREY95, ZHAN98, HENT00]. Eine Ausnahme bilden Daten, die von Boylan et al. und Osmola et al. veröffentlicht wurden [BOYL91, OSMO92]. Die Autoren wollen bei einer Ni2,2 At.\% P-Legierung $\mathrm{Ni}_{3} \mathrm{P}$-Ausscheidungen bereits nach Glühung bei $300{ }^{\circ} \mathrm{C}$ für 10 min beobachtet haben. Widersprüchlich ist auch, dass einer derselben Autoren in [MEHT95] $360{ }^{\circ} \mathrm{C}$ als Starttemperatur bei derselben Legierung angibt - bei deutlich längeren Auslagerungszeiten. Selbst dann, wenn im TEM und mit XRD kleinste Keime nicht detektierbar wären, so zeigen die TAP-Analysen eindeutig, dass es bei den niedrig P-haltigen Legierungen (3,6 und 5,9 At.\%) bis $\mathrm{zu}$ den Starttemperaturen der Umwandlung keine ausreichend hohen $\mathrm{P}$-Anreicherungen vorliegen, die als $\mathrm{Ni}_{3} \mathrm{P}$-Keime gelten könnten. Die Keimbildung setzt erst ab der Starttemperatur, und zwar bevorzugt in den Korngrenzen und Tripelpunkten, ein. Bei der 3,6 At.\% P-Legierung liegt diese bei $400{ }^{\circ} \mathrm{C}$. In diesem Zustand wurde eine 25 At.\%-ige P-Anreicherung beobachtet, die in einem Tripelpunkt der designierten Kornstruktur lokalisiert war. Es ist anzunehmen, dass es sich hierbei um einen $\mathrm{Ni}_{3} \mathrm{P}$-Keim handelt. Diese Beobachtung wird bestärkt durch die Untersuchungen von Osmola et al. an weiter fortgeschrittenen Stadien der Entmischung, bei denen $\mathrm{Ni}_{3} \mathrm{P}-\mathrm{Teilchen}$ bevorzugt in Korngrenzen und Tripelpunkten gefunden wurden [OSMO92].

Die Untersuchungen an den hoch P-haltigen 11,8 At.\% P-Proben zeigen auch, dass Phosphor sich anreichert. Die Proben sind deutlich heterogen. Bei isochroner Temperung bis $320{ }^{\circ} \mathrm{C}$ sind bereits Konzentrationen über 20 At.\% zu beobachten. Dies könnten bereits Vorläufer von Keimen sein. Die Untersuchungen nach längerer (isothermer) Glühung bei $320{ }^{\circ} \mathrm{C}$, bei denen die $\mathrm{Ni}_{3} \mathrm{P}$-Bildung nachgewiesen wurde, verdeutlichen dies. Unabhängig davon ist bei dieser Legierung die Untersuchung einer Morphologie der Anreicherungen prinzipiell sehr schwierig. Die mittlere Korngröße beträgt lediglich $3 \mathrm{~nm}$. Ein Analysvolumen mit 100 detektierten Atomen, welches sinnvoll für eine Konzentrationsermittlung herangezogen werden kann, hat immerhin eine Größe von 2,2 $\mathrm{nm}^{3}$. Das entspricht einer Kugel mit einem Durchmesser von 1,6 nm. Korngrößen und sinnvoll zu definierende Analysevolumina sind also ähnlich groß. Die Auflösung einer Morphologie auf dieser Längenskala ist daher sehr schwierig, zumal auch der zu erwartende Konzentrationshub relativ gering ist.

Die bei der $\mathrm{Ni}_{3} \mathrm{P}$-Bildung freigesetzten Wärmemengen, die mit der DSC bestimmt wurden, stimmen gut mit Angaben von Müller überein [MÜLL96], der bei 3,2 bzw 10,4 At.\% P-Gehalt Reaktionswärmen von 1,0 bzw. 2,3 kJ/mol angibt. ${ }^{18}$ Die Unsicherheit der Messwerte ist dabei relativ groß (20\%). Außerdem muss berücksichtigt werden, dass

\footnotetext{
${ }^{17}$ Die Angaben gelten für die verwendete Heizrate von $5^{\circ} \mathrm{C} / \mathrm{min}$.

${ }_{18}$ Müller gibt in seiner Arbeit für die Reaktionswärmen 17,4 bzw. 40,4 J/g an. Die Angaben im Text wurden auf die entsprechenden Molmengen umgerechnet.
} 
der Reaktion auch das parallel einsetzende Kornwachstum überlagert ist. Dessen Anteil an der Reaktionswärme ist jedoch unbestimmt.

Die erste, wiederum exotherme Umwandlung, die in den Thermogrammen zu beobachten ist, weist eine vergleichsweise ähnliche Reaktionswärme auf, wie die der $\mathrm{Ni}_{3} \mathrm{P}$ Bildung. Die Starttemperatur beträgt bei allen Legierungen ca. $120^{\circ} \mathrm{C}$, wobei die Peaks sehr breit sind und sich bis ca. $300{ }^{\circ} \mathrm{C}$ erstrecken. Auch hier gilt, dass die Messwerte relativ unsicher sind (40\%) und in gewissem Umfang Prozesse wie Relaxationen, z. B. Relaxationen in den Korngrenzen und die Ausheilung von Defekten, sowie ein geringfügiges Kornwachstum die Umwandlung überlagern. Die Untersuchung der Nanostruktur der angelassenen Proben zeigen allerdings, dass ein Umstand wesentlich für die Entwicklung des Gefüges ist: die Veränderung der Verteilung des Phosphors.

Die TAP-Untersuchungen an den beiden Legierungen Ni-3,6 At.\% P und Ni-5,9 At.\% P verdeutlichen unmittelbar die Entwicklungen der P-Verteilung. Die im Abscheidezustand beobachtete Übersättigung verringert sich mit steigender Temperatur und mit der Länge der Auslagerung, in dem der Phosphor sich an exponierten Stellen des Gefüges (den Korngrenzen) anreichert. Die in den Teilkapiteln 4.2.3.1 und 4.2.3.2 vorgestellten Ergebnisse verdeutlichen dies. Die Bereiche in denen sich der Phosphor anreichert sind schmale 2-dimensionale Zonen, die eine Dicke von ca. $1 \mathrm{~nm}$ aufweisen. Sie weisen die charakteristische 3-dimensionale Morphologie der Korngrenzen eines nanokristallinen Gefüges auf. Die mittleren Korngrößen stehen in guter Übereinstimmung mit den gefundenen Morphologien. Für die bei $320^{\circ} \mathrm{C}$ getemperten Ni3,6 At.\% P-Legierung beispielsweise wurde mit XRD ein mittlerer Korndurchmesser von 11,4 nm bestimmt. Die in Abb. 4.12 gezeigte designierte Kornstruktur korrespondiert offensichtlich sehr gut mit dieser Größe. Gleiches gilt auch für die Ni-5,9 At.\% PLegierung - mit noch kleinerer Korngröße von 9,2 nm -, die bis $385{ }^{\circ} \mathrm{C}$ ausgelagert wurde, s. Abb. 4.18. Es gibt keinen ersichtlichen Grund, dass die P-Anreicherungen nicht an den Korngrenzen lokalisiert sein sollten. Die TAP-Analysen zeigen daher eindeutig, dass der Phosphor durch Temperung - bis zu den kritischen Temperaturen der $\mathrm{Ni}_{3} \mathrm{P}$-Bildung - in den Korngrenzen segregiert. Damit konnte erstmals die Segregation in einem nanokristallinen Gefüge mit Hilfe von 3D-Atomsondentechnik unmittelbar nachgewiesen werden. Die von Hentschel et al. auf Basis von konventionellen APUntersuchungen abgeleitete Schlussfolgerung hinsichtlich einer P-Segregation in einer Ni-3,6 At.\% P-Legierung [HENT00] kann bestätigt werden. Auch bei dem höheren PGehalt von 5,9 At.\% ist die Segregation evident. Für die Ni-11,8 At.\% P-Legierung kann ein vergleichbarer Separationsmechanismus vermutet werden. Die zu erwartenden Strukturen bei Temperaturen von $300{ }^{\circ} \mathrm{C}$ oder weniger sind jedoch bei $3 \mathrm{~nm}$ Korngröße bereits am Rande der Auflösbarkeit.

Die Konzentrationsprofile durch die mit TAP analysierten Proben zeigen, dass sich die P-Konzentrationen in den angereicherten Korngrenzen und in den abgereicherten Körnern bei längerer Auslagerung und höheren Temperaturen - unterhalb der kritischen Temperaturen der $\mathrm{Ni}_{3} \mathrm{P}$-Bildung - auf näherungsweise konstante Werte einstellen: 14-15 At.\% P bzw. ca. 1 At.\% P. Und zwar unabhängig von der mittleren P-Konzentration. Die Breite der Segregationszonen beträgt ca. $1 \mathrm{~nm}$. Gleiche Werte wurden auch mit konventioneller AP für eine Ni-3,6 At.\% P-Legierung gefunden [HENT00]. Diese Beobachtung impliziert, aufgrund der notwendigen Massenerhaltung, einen direkten Zusammenhang zwischen der Korngrenzenfläche und der mittleren P-Konzentration. Weiter unten im Text wird diese Frage noch einmal aufgegriffen werden. 
Bei der Ermittlung der lokalen P-Konzentration in den Korngrenzen und der Breite der Segregationszonen muss allerdings beachtet werden, dass die gemessenen Werte auch abhängig sind von der Größe und Form des Testanalysevolumens (bzw. der Apertur bei der AP) und der Orientierung der Korngrenzen zur Analyserichtung. Eine ausführlichere Darlegung dieser Zusammenhänge ist in Anhang $\mathrm{C}$ nachzulesen. Unter optimierten Bedingungen, das heißt, mit kleinem Testanalysevolumen und bei Korngrenzen, die möglichst senkrecht zur Richtung der TAP-Analyse orientiert sind, kann die Breite der Segregationszone auf ca. 0,5 bis $1 \mathrm{~nm}$ abgeschätzt werden. Ein mittlerer Wert von 0,8 $\mathrm{nm}$ wurde in [FÄRB00] verwendet, um die Menge an Phosphor zu bestimmen, die in den Korngrenzen segregiert ist. Unter der vereinfachenden Annahme, dass die Dichte in der Korngrenze der von reinem Nickel entspricht, kann die Anzahl segregierter PAtome pro Korngrenzenfläche, der Korngrenzen-Exzess $\Gamma$, bestimmt werden. Es ist $\Gamma=\mathrm{c}_{\mathrm{gb}} \cdot \rho \cdot \Delta$, wobei $\rho$ die Dichte, $\mathrm{c}_{\mathrm{gb}}$ die Konzentration und $\Delta$ die Breite der Korngrenze ist. Bei einer Korngrenzenkonzentration von 15 At.\% P ergibt sich so ein Exzess in Höhe von $1,1 \times 10^{19}$ Atome $/ \mathrm{m}^{2}$.

Aufgrund der oben genannten Sachverhalte sind die Angaben über Breite und Höhe der Konzentrationspeaks verhältnismäßig unsicher. Der Korngrenzen-Exzess ist aber von seiner Definition her nicht an eine Dicke gekoppelt. In Kap. 4.2.4 wurde gezeigt wie die Exzesse präziser bestimmt werden können. An ausgewählten, gut rekonstruierbaren Korngrenzen wurden Analysen durchgeführt über größere Korngrenzenflächen (Durchmesser des Analysezylinders: $4 \mathrm{~nm}$ ), als dies im oben genannten Verfahren der Fall war. Mit Hilfe von Leiterdiagrammen konnte dann die Anzahl Atome in den Grenzflächen einfach ausgezählt werden. Die ermittelten Exzess-Größen für die beiden untersuchten Zustände der 3,6 At.\%-igen bzw. 5,9 At.\%-igen Legierung sind bei einem Wert von $\Gamma=9 \times 10^{18}$ Atome $/ \mathrm{m}^{2}$ identisch. In Bezug auf eine dichtestgepackte Lage von P-Atomen entspricht der Exzess 0,38 Monolagen Phosphor. Dabei wurde ein Atomradius von $0,1105 \mathrm{~nm}$ angenommen. Es ist bemerkenswert, dass die KorngrenzenExzesse identisch sind, obwohl die mittleren P-Konzentrationen unterschiedlich groß sind. Es wird daher vermutet, dass die Segregation hier einen Sättigungswert erreicht hat. D. h., dass alle energetisch günstigen oder alle zur Verfügung stehenden Plätze in den Korngrenzen besetzt sind. Für Ni-P-Legierungen sind keine Daten bekannt, die zu einem Vergleich herangezogen werden könnten. Bei Fe-P-Legierungen sind vergleichbar große Exzesse von Phosphor bekannt [HOND65, CAHN96-Kap. 13.2]. Der Sättigungswert für die Segregation von Phosphor in $\delta$-Eisen im thermodynamischen Gleichgewicht wird mit $6,5 \times 10^{18}$ Atome $/ \mathrm{m}^{2}$ angegeben.

\subsubsection{Thermische Stabilisierung durch Phosphor-Segregation}

Die Ergebnisse aus Kapitel 4.2 zeigen, dass sich die Korngrößen in den Ni-PLegierungen durch Wärmebehandlungen kaum ändern. Bei längerer Auslagerung für $1 \mathrm{~h}$ bei $320{ }^{\circ} \mathrm{C}$ bleiben die Korngrößen nahezu stabil (ausgenommen ist hier die $\mathrm{Ni}$ 11,8 At.\% P-Legierung, bei der bereits die $\mathrm{Ni}_{3} \mathrm{P}$-Bildung eingesetzt hat). Eine solche thermische Stabilität von Ni-P-Legierungen wird auch von anderen Autoren berichtet. Zhang et al. zeigen beispielsweise, dass sich die mittlere Korngröße einer Ni2,8 At.\% P-Legierung bei der Glühung mit $300{ }^{\circ} \mathrm{C}$ nach ca. $30 \mathrm{~min}$ bei ca. $15 \mathrm{~nm}$ stabilisiert [ZHAN98]. Auch in [BOYL91] wird berichtet, dass die Korngröße einer Ni2,3 At.\% P-Legierung bei der Auslagerung mit $300{ }^{\circ} \mathrm{C}$ zunächst von 5 auf $14 \mathrm{~nm}$ zunahm und sich anschließend über den Beobachtungszeitraum von mehr als $6 \mathrm{~h}$ stabilisierte. Die in dieser Arbeit vorgestellten Ergebnisse zeigen ferner, dass die thermische Stabilität nur so lange vorhanden ist bis die Bildung der $\mathrm{Ni}_{3} \mathrm{P}$-Phase einsetzt. 
Danach beginnt ein rasches Kornwachstum. Es stellt sich die Frage, was die Ursache für die thermische Stabilität der Legierungen ist.

Bedingt durch die kleinen Korngrößen sind die Korngrenzenflächen in den nanokristallinen Legierungen sehr groß. Unter der Annahme, dass die Körner kugelförmige Gestalt haben mit einem mittleren Durchmesser D, beträgt das Verhältnis von Korngrenzenfläche ${ }^{19} \mathrm{zu}$ Kornvolumen:

$$
\frac{A_{g b}}{V}=\frac{3}{D}
$$

Die Bedeutung der Grenzfläche kann an einem Beispiel verdeutlicht werden: Das Molvolumen von Nickel beträgt $6,59 \mathrm{~cm}^{3} / \mathrm{mol}$. Bei einer mittleren Korngröße von beispielsweise $6 \mathrm{~nm}$ ergibt sich daraus eine Korngrenzenfläche von ca $3300 \mathrm{~m}^{2} / \mathrm{mol}$. Setzt man die spezifische (freie) Korngrenzenenergie für Nickel mit ca. $\gamma_{0}=500 \mathrm{~mJ} / \mathrm{m}^{2}$ $\mathrm{an}^{20}$, so resultiert daraus in diesem Beispiel eine molare Korngrenzenenergie von immerhin $\gamma=1650 \mathrm{~J} / \mathrm{mol}$. Die treibende Kraft für das Kornwachstum $P$ ist daher groß. Nach [ATKI88] ist

$$
P \approx \frac{4 \gamma}{D}
$$

Für das Kornwachstum wird im allgemeinen folgender grundsätzlicher Ansatz gewählt [ATKI88]:

$$
\frac{d D}{d t} \propto \mu^{*} \cdot \frac{\gamma}{D},
$$

wobei $\mu^{*}$ die Mobilität ist. Die Stabilisierung von Korngrößen kann also nur erfolgen, wenn entweder die Mobilität stark eingeschränkt oder die thermodynamisch treibende Kraft stark abgesenkt wird.

Die nanostrukturellen Untersuchungen, die in den vorangegangenen Kapiteln vorgestellt wurden, zeigen, dass die thermische Stabilität mit der Sättigungs-Segregation des Phosphors in den Korngrenzen gekoppelt sein muss. Der gefundene SättigungsKorngrenzen-Exzess ermöglicht es, mit Hilfe eines einfachen geometrischen Modells den Zusammenhang zwischen der Korngröße in (getemperten) Ni-P-Legierungen und der mittleren P-Konzentration der Legierungen vorherzusagen. Auf Basis der Ergebnisse in Kap. 4.2.4 sei angenommen, dass pro Korngrenzenfläche ein konstante Menge Phosphor, entsprechend einem Exzess von $\Gamma_{0}=9 \times 10^{18} \mathrm{~m}^{-2}$, segregiert. Die Anzahl der $\mathrm{P}$-Atome in der gesamten (molaren) Korngrenzenfläche $\mathrm{A}_{\mathrm{gb}}$ beträgt dann

$$
N_{g b}^{P}=\Gamma_{0} \cdot A_{g b} .
$$

Der verbleibende P-Gehalt in den Körnern sei als konstant angenommen. Mit Bezug auf die TAP-Messungen sei dieser mit $c_{g}=1$ At. $\%$ angesetzt. Die Anzahl der P-Atome in den Körnern pro Mol Legierung ergibt sich dann mittels

$$
N_{g}^{P}=c_{g}\left(N_{A}-N_{g b}^{P}\right),
$$

\footnotetext{
${ }^{19}$ Man beachte, dass die Korngrenze zu gleichen Teilen zwei Körnern zuzurechnen ist.

${ }^{20}$ Dieser Wert wurde anhand der DSC-Daten zum Kornwachstum von nanokristallinem reinem Nickel von U. Klement et al. [KLEM95] und Gl. 4.2 bestimmt.
} 
wobei $\mathrm{N}_{\mathrm{A}}$ die Avogadro-Konstante ist. Für die mittlere P-Konzentration in einem Mol Legierung folgt daher

$$
c_{0}=\frac{N_{g}^{P}+N_{g b}^{P}}{N_{A}} .
$$

Die Avogadro-Konstante lässt sich auch über das Molvolumen $\mathrm{V}_{\mathrm{M}}$ und die Atomdichte $\rho$ ausdrücken. Es ist $\mathrm{N}_{\mathrm{A}}=\mathrm{V}_{\mathrm{M}} \cdot \rho$. Dabei sei die Atomdichte als konstant, mit dem Wert reinen Nickels $\left(\rho=9,14 \times 10^{28}\right.$ Atome $\left./ \mathrm{m}^{3}\right)$ angenommen. Daraus folgt aus den Gleichungen 4.5, 4.6 und 4.7:

$$
\begin{aligned}
c_{0} & =\frac{c_{g}\left(V_{M} \rho-\Gamma_{0} A_{g b}\right)+\Gamma_{0} A_{g b}}{V_{M} \rho} \\
& =c_{g}+\left(1-c_{g}\right) \cdot \frac{\Gamma_{0} A_{g b}}{V_{M} \rho}
\end{aligned}
$$

Durch Einsetzen von Gleichung 4.2 ergibt sich dann für die mittlere Korngröße:

$$
D=\frac{3 \cdot \Gamma_{0}}{\rho} \cdot \frac{1-c_{g}}{c_{0}-c_{g}} .
$$

Für $\mathrm{c}_{\mathrm{g}}<<1$ lässt sich die Gleichung noch weiter vereinfachen zu

$$
D \approx \frac{3 \cdot \Gamma_{0}}{\rho} \cdot \frac{1}{c_{0}-c_{g}} .
$$

Gleichung 4.10 zeigt den zu erwartenden Zusammenhang zwischen mittlerem P-Gehalt $\mathrm{c}_{0}$ und der mittleren Korngröße D unter der Annahme, dass die Kornstruktur sich bei einer definierten Sättigungs-Segregation, die durch den konstanten Exzess $\Gamma_{0}$ repräsentiert ist, und bei einem konstanten verbleibenden P-Gehalt in den Körnern $\mathrm{c}_{\mathrm{g}}$ stabilisiert. Es sei bemerkt, dass diese Formel ausschließlich messbare physikalische Größen beinhaltet, also keine Fitparameter. In Abb. 4.24 ist der Verlauf der Kurve gemäß Gl. 4.10 zusammen mit den in dieser Arbeit ermittelten Korngrößen der Ni-P-Legierungen im Abscheidezustand und im angelassenen Zustand nach Auslagerung bei $320{ }^{\circ} \mathrm{C} \backslash 1 \mathrm{~h}$ dargestellt. Außerdem sind die Daten aus der Literatur mit eingezeichnet.

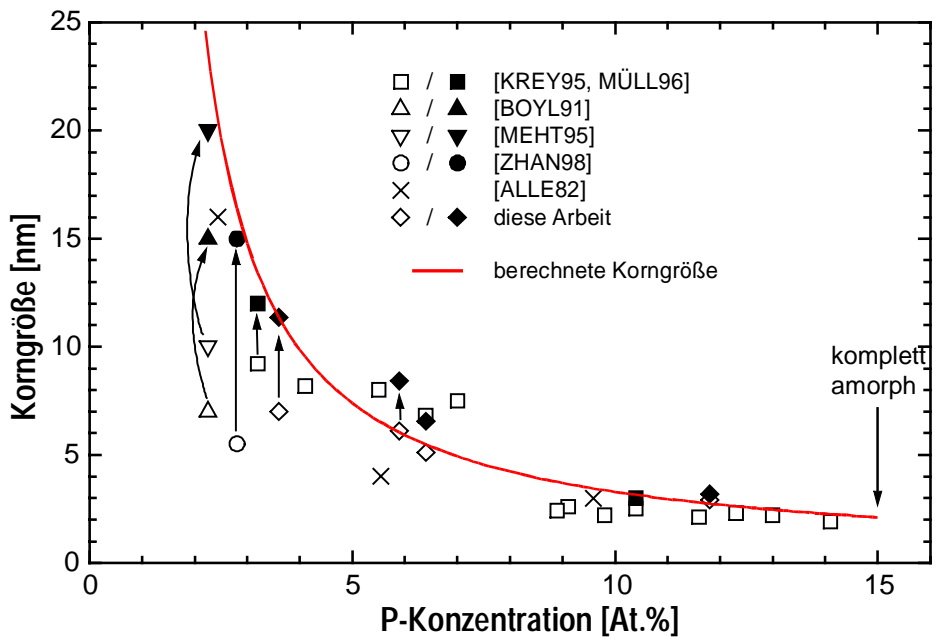

Abb. 4.24 Die Korngröße von NiP-Legierungen als Funktion des PGehalts. Offene Symbole repräsentieren den Abscheidezustand, ausgefüllte den Zustand nach Temperung bei 300 oder $320{ }^{\circ} \mathrm{C}$. Die eingezeichnete Kurve beschreibt die zu erwartenden Korngrößen bei einer Stabilisierung durch eine konstante Sättigungs-Segregation $\Gamma_{0}=9 \times 10^{18} \mathrm{~m}^{-2}$, s. Text und Gleichung 4.10 . 
Die gezeigte Kurve gibt recht gut die experimentell gefundenen Korngrößen wieder. Gut $\mathrm{zu}$ erkennen ist, dass für niedrige P-Gehalte die kleineren Korngrößen im Abscheidezustand nach Temperung bei 300 bis $320{ }^{\circ} \mathrm{C}$ in etwa auf die postulierte Größe anwachsen. Bei größeren P-Gehalten sind die Korngrößen von Anfang an klein und das anschließende Wachstum fällt auch entsprechend gering aus. In [FÄRB00] wurde anhand von konstanten Korngrenzenbreiten und Korngrenzenkonzentrationen eine in ihrem Verlauf ähnliche Kurve abgeleitet, die den Zusammenhang $\mathrm{D}\left(\mathrm{c}_{0}\right)$ auch gut beschreiben kann. Der Vorteil von Gl. 4.10 ist jedoch, dass die verwendeten Größen genauer bestimmt werden können. Insbesondere der Exzess, der von der Korngrenzenbreite unabhängig ist. Unabhängig davon bleibt festzuhalten, dass die Stabilisierung der Kornstrukturen unmittelbar mit der Sättigungs-Segregation verbunden ist.

Das Kornwachstum könnte, wie aus Gl. 4.4 abzulesen ist, durch starke Einschränkung der Kinetik verlangsamt und behindert werden. In der Literatur werden die Ursachen für die thermische Stabiliät von Ni-P-Legierungen teilweise kontrovers diskutiert und mehrere Mechanismen der Hemmung genannt. Boylan et al. [BOYL91] und Zhang et al. [ZHAN98] vermuten, dass im wesentlichen $\mathrm{Ni}_{3} \mathrm{P}$-Ausscheidungen die Korngrenzenbewegung durch den Zener-Effekt behindern, wobei die Korngrenzenfläche, und die damit verbundene Korngrenzenenergie, durch eine darin liegende Ausscheidung einer zweiten Phase verringert wird. Die Stabilisierung der Korngrößen wird jedoch bereits bei Temperaturen beobachtet, bei denen die Autoren konkret keine $\mathrm{Ni}_{3} \mathrm{P}$-Ausscheidungen nachweisen. Wie bereits in Kap. 4.3 diskutiert, zeigen die Ergebnisse dieser Arbeit keine Hinweise auf $\mathrm{Ni}_{3} \mathrm{P}$-Ausscheidungen in den Stadien der thermischen Stabilisierung. Im Gegenteil, die Stabilität verschwindet mit Bildung der $\mathrm{Ni}_{3} \mathrm{P}-\mathrm{Ph}$ ase. Ein Zener-Effekt als Ursache für die Stabilität kann also ausgeschlossen werden.

Ein weiterer Aspekt wird von mehreren Autoren [OSMO92, ZHAN98] angeführt: der Einfluss von Tripelpunkten. In [PALU90] wird eine einfache Abschätzung über den Volumenbruchteil von Tripelpunkten in nanokristallinen Strukturen vorgenommen. Dieser steigt merklich im Vergleich zum restlichen Korngrenzenvolumen bei Korngrößen kleiner $10 \mathrm{~nm}$. Der erwogene Einfluss von Tripelpunkten auf die Stabilität der Kornstruktur, Osmola et al. sprechen von einem ,triple junction drag“-Effekt [OSMO92], ist jedoch unbekannt und nicht näher spezifiziert.

Als mögliches kinetisches Hemmnis des Kornwachstums wird außerdem der SoluteDrag-Effekt, d. h. das Bremsen der Korngrenzbewegung durch eine gelöste Komponente (Phosphor) und ihre Wechselwirkung mit der Korngrenze, genannt [MEHT95]. Mehta et al. machen eine grobe Abschätzung der rücktreibenden Kraft nach [HAES78], $\mathrm{P}=4 \mathrm{c}_{0} \gamma / \mathrm{r}_{\text {Atom }}{ }^{21}$, in dem das einzelne P-Atom quasi als Ausscheidung (mit Radius gleich Atomradius $\mathrm{r}_{\text {Atom }}$ ) behandelt wird. Das Ergebnis dieser Überlegung ist, dass bereits Phosphor in homogen übersättigter Lösung (hier mit 2,3 At.\%) eine genügend große rücktreibende Kraft ausüben würde, die ausreichte, die Kornstuktur bei weniger als 10 nm Korngröße stabil zu halten. Daraus wird gefolgert, dass Phosphor vermutlich in homogen übersättigter Lösung vorläge. Die von Mehta et al. vorgenommenen quantitativen Abschätzungen sind jedoch nach Angabe des Autors, auf den Bezug genommen wird, explizit nicht zulässig, da die P-Atome im Unterschied zu Teilchen beweglich sind [HAES78-Kap. 2.3.2]. Der propagierte Solute-Drag-Effekt von homogen gelöstem Phosphor ist daher nicht nachvollziehbar. Dies gilt insbesondere auch aufgrund der

\footnotetext{
${ }^{21}$ Von Haessner [HAES78] wird im Original ein um den Faktor 2 kleinerer Wert für P angegeben.
} 
Ergebnisse dieser Arbeit, die eindeutig zeigen, dass Phosphor in den Korngrenzen segregiert.

Nichtsdestotrotz ist es denkbar, dass in einem gewissen Umfang durch einen SoluteDrag-Effekt das Kornwachstum behindert wird. Die theoretischen Grundlagen für die Beschreibung des Effektes wurden von Cahn gegeben [CAHN62]. Bei kleinen Geschwindigkeiten $(\mathrm{V})$ der Korngrenzenbewegung gilt für die rücktreibende Kraft $P_{i}$ :

$$
P_{i}=4 \rho \cdot c_{0} V k T \int_{-\infty}^{+\infty} \frac{\sinh ^{2}(E(x) / 2 k T)}{D^{*}(x)} d x,
$$

wobei $\rho$ die Atomdichte, $\mathrm{c}_{0}$ die P-Konzentration, $\mathrm{T}$ die Temperatur, $\mathrm{E}(\mathrm{x})$ die Wechselwirkung des Phosphors mit der Korngrenze und D*(x) der Diffusionskoeffizient des Phosphors ist. Die Koordinate $x$ beschreibt den Abstand von der Grenzfläche. Bei starker Tendenz zu Segregation an der Grenzfläche, d. h. E(x) ist negativ und groß, und bei als konstant angenommenem Diffusionskoeffizienten kann Gl. 4.11 für den stationären Fall, in dem treibende Kraft gleich rücktreibender Kraft ist, reduziert werden auf

$$
V=\frac{P D^{*}}{k T \Gamma}
$$

wobei $P$ die treibende Kraft, $D^{*}$ der Bulk-Diffusionkoeffizient und $\Gamma$ der PhosphorKorngrenzen-Exzess ist. Aus Gl. 4.12 werden die grundlegenden Zusammenhänge ersichtlich. Bei geringer treibender Kraft und geringer Beweglichkeit des Phosphors, sowie bei großem Exzess wäre das Kornwachstum stark gehemmt. Die treibenden Kräfte sind nach Gl. 4.3 groß. Über den Diffusionskoeffizienten von Phosphor in Nickel ist nichts bekannt. Allerdings kann aus den Experimenten geschlossen werden, dass Phosphor bei Temperaturen von $320{ }^{\circ} \mathrm{C}$ innerhalb der Zeitgrößenordnung $1 \mathrm{~h}$ auf einer 5-10 nm Längenskala bereits beweglich ist. Bei der Legierung mit 5,9 At.\% P änderte sich die mittlere Korngröße nach Auslagerung für $1 \mathrm{~h}$ bei $320^{\circ} \mathrm{C}$ von 6,1 auf $8,4 \mathrm{~nm}$. Der in den Korngrenzen segregierte Phosphor kann also nicht durch die fortschreitenden Korngrenzen ,,aufgesammelt“ worden sein, sondern muss größtenteils durch die eigene Beweglichkeit dahin gelangt sein. ${ }^{22}$ Daraus lässt sich grob ein Diffusionskoeffizient von $\mathrm{D}^{*} \approx 1 \times 10^{-20} \mathrm{~m}^{2} / \mathrm{s}$ abschätzen. Bei einer Korngröße von $10 \mathrm{~nm}$, einer Korngrenzenenergie von $500 \mathrm{~mJ} / \mathrm{m}^{2}$ und einem Korngrenzen-Exzess von $9 \times 10^{18} \mathrm{~m}^{-2}$ ergäbe sich also nach Gl. 4.3 und 4.12 eine Korngrenzengeschwindigkeit von ca. $\mathrm{V} \approx 100 \mathrm{~nm} / \mathrm{h}$. Das beobachtete Kornwachstum ist im Vergleich dazu allerdings zwei Größenordnungen kleiner. Die Berechnungen deuten also darauf hin, dass der Solute-Drag-Effekt alleine nicht für die thermische Stabilität der Ni-P-Legierungen verantwortlich sein kann. Eine rein kinetisch bedingte Hemmung des Kornwachstums scheint bei Ni-P-Legierungen nicht vorzuliegen.

Aus Gl. 4.4 war abzulesen, dass neben der Kinetik des Kornwachstums auch die thermodynamisch treibende Kraft von entscheidender Bedeutung ist. Durch die Absenkung der spezifischen Korngrenzenenergie würde die treibende Kraft verringert

\footnotetext{
22 Michels et al. [MICH99] stellen in einer neuen Veröffentlichung fest, dass in nanokristallinen Materialien das „Aufsammeln“ der gelösten Spezies durch die fortschreitenden Korngrenzen der Regelfall ist. Daraus wird dann ein Zusammenhang zwischen Korngröße und Segregation abgeleitet und für weitere Modellierungen verwendet. Die hier gemachte Beobachtung zeigt, dass diese Annahme für Ni-PLegierungen zumindest nicht gültig ist.
} 
und das Kornwachstum verlangsamt. Die Segregation des Phosphors in den Korngrenzen kann zu dieser Absenkung führen. In der grundlegenden Theorie der Segregation wird für die Beschreibung des Sachverhaltes von der Gibbs'schen Adsorbtionsisotherme ausgegangen. Sie besagt, dass jede gelöste Komponente in einer Flüssigkeit oder einem Festkörper, die die Oberflächenspannung absenkt, einen positiven Exzess, also eine höhere Konzentration an der Oberfläche, hat [CAHN96Kap. 5.9.1]. Es gilt:

$$
\Gamma_{B}=-\frac{1}{k T}\left(\frac{\partial \sigma^{*}}{\partial \ln a_{B}}\right)_{T},
$$

wobei $\Gamma_{B}$ der Exzess der Komponente B an der Oberfläche, T die Temperatur, $\sigma^{*}$ die Oberflächenspannung und $\mathrm{a}_{\mathrm{B}}$ die Aktivität der Komponente B ist. Dieses Konzept wird auf innere Grenzflächen übertragen, indem die Oberflächenspannung durch die Grenzflächenenergie ausgetauscht wird. Außerdem wird angenommen, dass die Aktivität proportional zur Konzentration $\mathrm{x}_{\mathrm{B}}$ ist $\left(\mathrm{a}_{\mathrm{B}} \propto \mathrm{x}_{\mathrm{B}}\right.$, Henry's Gesetz). Dies ist erfüllt für kleine Konzentrationen $\left(\mathrm{x}_{\mathrm{B}}<<1\right)$. Es folgt daraus das Gibbs'sche Adsorbtionstheorem [SUTT95, CAHN96-Kap. 13.2]:

$$
\left(\frac{\partial \gamma}{\partial \ln x_{B}}\right)_{T}=-k T \cdot \Gamma_{B}
$$

Die Gleichung zeigt, dass mit einem positiven Exzess eine Absenkung der Korngrenzenenergie bei zunehmenden mittleren Konzentrationen verbunden ist. Von Hondros et al. [HOND65] wurden beispielsweise im System Eisen-Phosphor durch Messung von Grenzflächenenergien Absenkungen von 800 auf 400 mJ/m² innerhalb eines Konzentrationsintervalls von 0,1 bis 1,0 At. $\% \mathrm{P}$ und bei Temperaturen von $1450{ }^{\circ} \mathrm{C}$ bestimmt. Über Gl. 4.14 ist damit ein Exzess von $6,5 \times 10^{18} \mathrm{~m}^{-2}$ verbunden.

Bezogen auf die Ni-P-Legierungen heißt dies, dass die Korngrenzenenergie bei einer Sättigungssegregation $\Gamma_{0}=9 \times 10^{18} \mathrm{~m}^{-2}$, wie sie in dieser Arbeit gefunden wurde, und bei Temperaturen von $320{ }^{\circ} \mathrm{C}$ über eine Zehnerpotenz in der Konzentration um ca. 170 $\mathrm{mJ} / \mathrm{m}^{2}$ abgesenkt wird. Wie beim Eisen-Phosphor kann also auch hier die Korngrenzenenergie deutlich abgesenkt werden. Wie groß dieser Effekt letztendlich ist bleibt noch unklar. Es ist jedoch nicht prinzipiell ausgeschlossen, dass ein Großteil der Korngrenzenenergie durch die Segregation abgebaut wird.

Zusammenfassend ergibt sich also folgendes Bild bezüglich der thermischen Stabilität der Ni-P-Legierungen: Die Stabilität ist verbunden mit der P-Segregation in den Korngrenzen. Die $\mathrm{Ni}_{3} \mathrm{P}$-Phasenbildung spielt dabei nur insofern eine Rolle, dass die Stabilität zusammenbricht, sobald sie einsetzt. Die Segregation stellt einen Sättigungswert dar, d. h. alle energetisch günstigen oder zur Verfügung stehenden Plätze in den Korngrenzen sind belegt. Durch die Segregation ist einerseits eine Einschränkung der Kinetik des Kornwachstums und andererseits eine deutliche Absenkung der treibenden Kraft, sprich der Korngrenzenenergie, verbunden. Die Stabilisierung von unterschiedlichen Korngrößen bei unterschiedlichen P-Gehalten und dennoch gleichem Exzess deutet darauf hin, dass die treibenden Kräfte, sprich die Korngrenzenergien, in diesen Zuständen sehr klein, wenn nicht gar verschwindend sind. In diesem Fall wäre die Stabilisierung das Ergebnis der Einstellung eines metastabilen Gleichgewichts. 


\section{Zusammenfassung}

Gegenstand dieser Arbeit war die Untersuchung der nanostrukturellen Entwicklung von nanokristallinen (mittlere Korngrößen kleiner $10 \mathrm{~nm}$ ), binären Fe-Ag- und Ni-P-Legierungen nach Wärmebehandlungen. Beide Materialien zeigen eine ungewöhnliche thermische Stabilität der nanokristallinen Kornstruktur. Bei Temperung stagniert das Kornwachstum nach einer anfänglichen geringfügigen Kornvergröberung. Die Verteilung der Legierungselemente in den Nanostrukturen der Materialien hat dabei einen wesentlichen Einfluss. Es wurden daher Untersuchungen zur Charakterisierung der Mikro- und Nanostrukturen vorgenommen. Entsprechend der feinen Struktur der Materialien wurde die höchstauflösende Analyse mit einer modernen 3D-Atomsonde, der Tomographische Atomsonde (TAP), durchgeführt.

Die Fe-Ag-Legierungen wurden mit Hilfe der gepulsten Laserdeposition (PLD) als dünne Schichten deponiert. Für die Untersuchung mit der TAP wurden diese auf speziell präparierte Wolfram-Substratspitzen aufgebracht. Die TAP-Analysen zeigten, dass die Fe-Ag-Legierungen auf der Fe-reichen Seite weit über ihre Löslichkeitsgrenze $(0,022$ At.\% Ag) hinaus übersättigt werden können. Es wurde der unmittelbare Nachweis erbracht, dass die Legierungen im ,wie hergestellt“ Zustand bis zu AgGehalten von 3 At.\% homogen sind. Im Vergleich zu Schichten, die parallel auf planaren Substraten deponiert wurden, wurden dabei große Diskrepanzen hinsichtlich der mittleren Ag-Gehalte gefunden. Während planar bis zu 28 At.\% Ag deponiert wurde, betrug der Ag-Gehalt der Schichten auf den Spitzen lediglich bis zu 3 At.\%. Auch die Kornstrukturen der Schichten wiesen z. T. drastische Unterschiede auf. Während die planaren Schichten nanokristallin sind (Korngrößen kleiner $10 \mathrm{~nm}$ ), wurden auf den Spitzen z. T. einkristalline, auf dem W-Substrat epitaktisch gewachsene Schichten beobachtet. Die wesentliche Ursache für die differierenden Schichteigenschaften ist ein winkelabhängiges präferentielles Sputtern des Silbers bei der PLD, wie es sich durch selbst durchgeführte TRIM-Simulationen nachweisen lässt. Die mit PLD hergestellten Fe-Ag-Schichten auf Spitzen sind also mit denen auf planaren Substraten nicht vergleichbar. Eine gezielte Untersuchung der nanostrukturellen Entwicklung der Legierungen (auf Spitzen) parallel zu planaren Schichten ist daher nicht sinnvoll. Wärmebehandlungen und röntgenographische Untersuchungen an planaren Schichten [STÖR98] deuten darauf hin, dass ein Großteil des Silbers in den Korngrenzen segregiert und damit die thermische Stabilität verursacht. Rein rechnerisch wurde daraus ein maximal möglicher Ag-Korngrenzen-Exzess von 3,7×10 ${ }^{19} \mathrm{~m}^{-2}$ ermittelt.

Die Ni-P-Legierungen wurden chemisch abgeschieden. In diesem Fall konnten alle Proben direkt aus den (dickeren) Schichten heraus präpariert werden. Es wurden Schichten mit P-Gehalten von 3,6, 5,9, 6,4 und 11,8 At.\% hergestellt, die Korngrößen zwischen 7 und $3 \mathrm{~nm}$ aufwiesen. Im Abscheidezustand wurde eine starke Übersättigung der Nickel-Körner mit Phosphor beobachtet. Wärmebehandlungen bis zu $320^{\circ} \mathrm{C}$ führen zu einer leichten Vergröberung der Kornstrukturen, die sich anschließend bei längerer Auslagerungsdauer stabilisiert. Bei höheren Temperaturen bis $\mathrm{zu} 400^{\circ} \mathrm{C}$ setzt die Bildung der $\mathrm{Ni}_{3} \mathrm{P}$-Phase ein. Dies geschieht bevorzugt an Korngrenzen und in Tripelpunkten. Damit verbunden ist dann der Beginn eines rapiden Kornwachstums. Es wurde gezeigt, dass die Ursache für die thermische Stabilisierung in der Segregation des Phosphors in den Korngrenzen besteht. Die Segregation in der nanokristallinen Struktur konnte unmittelbar nachgewiesen und genauer bei 3,6 und 5,9 At.\%-igen Legierungen untersucht werden. Es zeigt sich, dass der P-Gehalt in den Korngrenzen unabhängig von 
der mittleren Konzentration einen Sättigungswert anstrebt. Der Korngrenzen-Exzess konnte direkt durch das Auszählen der P-Atome in den Grenzflächen aus den Analysen bestimmt werden. Er beträgt $\Gamma_{0}=9 \times 10^{18} \mathrm{~m}^{-2}$. Auf Basis des konstanten Exzesses wurde ein parameterfreier Zusammenhang zwischen den mittleren P-Konzentrationen und den stabilisierten Korngrößen, der sehr gut mit experimentellen Ergebnissen übereinstimmt, abgeleitet. Als Ursachen für die thermische Stabilität werden kinetische und thermodynamische Hemmung diskutiert. Vermutungen anderer Autoren hinsichtlich eines mögliche Zener-Effektes durch $\mathrm{Ni}_{3} \mathrm{P}$-Ausscheidungen oder eines Solute-Drag-Effektes (verursacht durch homogen gelöste P-Atome) können eindeutig widerlegt werden. Die kinetische Hemmung der Korngrenzenbewegung liefert dennoch einen Beitrag zur Stabilisierung. Allerdings scheint der thermodynamische Einfluss, d. h. die deutliche Absenkung der Korngrenzenenergie durch die Segregation und damit verbunden die Absenkung der treibenden Kraft für das Kornwachstum, zu überwiegen. Die thermische Stabilisierung der Körner kann daher auch als Einstellung eines metastabilen Gleichgewichtes aufgefasst werden, sofern die treibende Kraft des Kornwachstums auf Null abgesenkt würde.

Nanokristalline Materialien allgemein sind durch den sehr großen Anteil an Korngrenzenfläche pro Volumeneinheit geprägt. Ihr Einfluß auf makroskopische Eigenschaften und physikalische Prozesse ist daher wesentlich. Bei nanokristallinen binären Legierungen wie Eisen-Silber und Nickel-Phosphor, die in ihren Ausgangszuständen stark übersättigt sind, spielt die Segregation in den Korngrenzen die dominierende Rolle bei der Entwicklung der Nanostrukturen unter Wärmeeinfluss. Erst die moderne hochauflösende Analytik erlaubt diese Vorgänge besser zu verstehen. 


\section{Symbolverzeichnis}

\section{Abkürzungen:}

3DAP 3D-Atomsonde

AP Atomsonde

EDX Energiedispersive Röntgenanalyse

EMS Elektronenstrahlmikrosonde

FIM Feldionenmikroskop

MCP Mikrokanalplatte

PLD gepulste Laserdeposition

TAP Tomographische Atomsonde

TEM Transmissionselektronenmikroskop

TRIM Transport of Ions in Matter, Software

UHV Ultrahochvakuum

XRD Röntgendiffraktometrie

\section{Mathematische Symbole, latein:}

a

$\mathrm{A}_{\mathrm{gb}} \quad$ Korngrenzenfläche

b Bildkompressionsfaktor

$\mathrm{c}_{0} \quad$ mittlere Konzentration

$c_{g} \quad$ Konzentration in einem Korn

D Korndurchmesser

D* Diffusionskoeffizient

$\mathrm{E}_{\mathrm{d}} \quad$ Kritische Verrückungsenergie (Displacement-Energy)

$\mathrm{E}_{1} \quad$ Gitterplatz-Bindungsenergie (Lattice-Binding-Energy)

$\mathrm{E}_{\mathrm{s}} \quad$ Oberflächen-Bindungsenergie (Suface-Binding-Energy)

F elektrische Feldstärke

$\mathrm{F}_{\mathrm{f}} \quad$ Feldverdampfungsfeldstärke

k Geometriefaktor

L Fluglänge

P thermodynamisch treibende Kraft

$\mathrm{r}_{\mathrm{t}} \quad$ Spitzenradius

$\mathrm{U}_{\mathrm{DC}} \quad$ Gleichspannung, Grundspannung

$\mathrm{U}_{\mathrm{P}} \quad$ Pulsspannung 


$\begin{array}{ll}\mathrm{V} & \text { Korngrenzengeschwindigkeit } \\ \mathrm{V}_{\mathrm{M}} & \text { Molvolumen } \\ \mathrm{Y} & \text { Sputterausbeute } \\ \mathrm{Y}_{\mathrm{bs}} & \text { Rückstreuanteil } \\ \mathrm{Y}_{\mathrm{eff}} & \text { effektive Sputterausbeute }\end{array}$

\section{Mathematische Symbole, griechisch:}

$\begin{array}{ll}\alpha & \text { Pulsverhältnis } \\ \gamma & \text { Korngrenzenenergie } \\ \Gamma_{0} & \text { Korngrenzen-Exzess } \\ \lambda & \text { Wellenlänge } \\ \mu & \text { chemisches Potential } \\ \mu^{*} & \text { Mobilität } \\ \sigma & \text { Standardabweichung } \\ \Theta & \text { Bragg-Winkel }\end{array}$




\section{Literaturverzeichnis}

[ALKA96] Al-Kassab, T., Macht, M.-P., Naundorf, V., Wollenberger, H., Chambreland, S., Danoix, F. und Blavette, D., Appl. Surf. Sci., 94 / 95, 306-312, 1996.

[ALLE82] Allen, R. M. und VanderSande, J. B., Scripta metall., 16, 1161-1164, 1982.

[ATKI88] Atkinson, H. V., Acta metall., 36 (3), 469-491, 1988.

[BAS 95] Bas, P., Bostel, A., Deconihout, B. und Blavette, D., Appl. Surf. Sci., 87/88, 298-304, 1995.

[BAS-97] Bas-Chambreland, P., Reconstruction tridimensionelle des images de Tomographie Atomique, Dissertation, Universität Rouen, Frankreich, 1997.

[Benn95] Bennett, T. D., Grigoropoulos, C. P. und Krajnovich, D. J., J. Appl. Phys. 77 (2), 849-864, 1995.

[BERn69] Bernardini, J., Combe-Brun und A., Cabane, J., Comptes rendus des seances de l'Academie des Sciences Paris, Serie C, 269, 287-289, 1969.

[BIER80] Biersack, J. P. und Haggmark, L., Nucl. Instr. and Meth., 174, 257, 1980.

[BLAV93] Blavette, D., Deconihout, B., Bostel, A., Sarrau, J. M., Bouet, M. und Menand, A., Rev. Sci. Instrum., 64 (10), 2911-2919, 1993.

[BLAV96] Blavette, D., Duval, P., Letellier, L. und Guttmann, M., Acta mater., 44 (12), 4995-5005, 1996.

[BOER88] Boer, F. R. de, Boom, R., Mattens, W. C. M., Miedema, A. R. und Niessen A. K., Cohesion in Metals - Transition Metal Alloys, North-Holland Physics Publishing, Amsterdam, 1988.

[BOWK70] Bowkett, K. M. und Smith, D. A., Field-Ion Microscopy, North-Holland Publishing Company, Amsterdam, 1970.

[BOYL91] Boylan, K., Ostrander, D., Erb, U., Palumbo, G. und Aust, K. T., Scri. Metall. et Mater., 25, 2711-2716, 1991.

[BRON91] Bronstein, I. N. und Semendjajew, K. A., Taschenbuch der Mathematik, 25. Aufl., B. G. Teubner Verlagsgesellschaft, Stuttgart, 1991.

[CAHN62] Cahn, J. W., Acta Metall., 10, 789-798, 1962.

[CAHN96] Cahn, R. W. und Haasen, P., Ed., Physical Metallurgy, Elsevier Science B.V., North-Holland, 1996.

[CHRI94] Chrisey, D. B. und Hubler, G. H., Ed., Pulsed Laser Deposition of Thin Films, Wiley, New York, 1994.

[CULL67] Cullity, B. D., Elements of X-Ray Diffraction, Addison-Wesley Publishing Company, Inc., Reading, 1967. 
[CULL67] Cullity, B. D., Elements of X-Ray Diffraction, Addison-Wesley Publishing Company, Inc., Reading, 1967.

[DECO94] Deconihout, B., Bostel, A., Bas, P., Chambreland, S., Letellier, L., Danoix, F. und Blavette, D., Appl. Surf. Sci., 76/77, 145-154, 1994.

[DYER89] Dyer, P. E., Appl. Phys. Lett., 55, 1630-1632, 1989.

[FÄHL97] Fähler, S., Störmer, M. und Krebs, H.-U., Appl. Surf. Sci, 109-110, 433, 1997.

[FÄHL98] Fähler, S., Der Einfluss der kinetischen Energie der Ionen auf das Wachstum laserdeponierter metallischer Schichten und Schichtpakete, Dissertation, Universität Göttingen und Shaker-Verlag, Aachen, 1998.

[FÄRB 0 0] Färber, B., Cadel, E., Menand, A., Schmitz, G. und Kirchheim, R., Acta Mater., 48 (3), 789-796, 2000.

[GAVI91] Gavigan, J. P., Hadjipanayis, G. C. und Prinz, G. A., Science and Technology of Nanostructured Magnetic Materials. Proceedings of a NATO Advanced Study Institute., 81-89, New York, 1991.

[GeBe92] Geber, G. P., Al-Kassab, T., Isheim, D., Busch, R. und Haasen, P., Z. Metallkd., 83, 449-456, 1992.

[GLEI89] Gleiter, H., Progress in Mater. Sci., 33, 223-315, 1989.

[GRAH65] Graham, A. H., Lindsay, R. W. und Read, H. J., J. Electrochem. Soc., 112 (4), 401-413, 1965.

[HAES78] Haessner, F. (Ed.), Recrystallization of Metallic Materials, Dr. Riederer Verlag, Stuttgart, 1978.

[HENT0 0] Hentschel, T., Isheim, D., Kirchheim, R., Müller, F. und Kreye, H., Acta. Mater., 48 (4), 933-941, 2000.

[HOND65] Hondros, E. D., Proc. Roy. Soc. A, 286, 479-498, 1965.

[IBM 96] IBM Corp., http://www.research.ibm.com/ionbeams/SRIM/SRIMA.HTM, 1996.

[INGE94] Ingen, R. P. van, Fastenau, R. H. J. und Mittemeijer, E. J., Phys. Rev. Lett. 72 (19), 3116-3119, 1994.

[KITT93] Kittel, C., Einführung in die Festkörperphysik, 10. Aufl., R. Oldenbourg Verlag, München, 1993.

[KEST99] Kesten, P., persönliche Mitteilungen, 1999.

[KLEM95] Klement, U., Erb, U., El-Sherik, A. M. und Aust, K. T., Mater. Sci. Engin. A, 203, 177-186, 1995. 
[KOOL92] Kools, J. C. S., Baller, T. S., De Zwart, S. T. und Dieleman, J., J. Appl. Phys., 71 (9), 4547-4556, 1992.

[KReB93] Krebs, H.-U. und Bremert, O., Appl. Phys. Lett., 62 (19), 2341-2343, 1993.

[KREB95] Krebs, H.-U., Bremert, O., Störmer, M. und Luo, Y., Appl. Surf. Sci., 86, 90-94, 1995.

[KREB97] Krebs, H.-U., Intern. J. Non-Equilibrium Processing, 10, 3-24, 1997.

[KREB99] Krebs, H.-U., private Mitteilungen, 1999.

[KREY86] Kreye, H., Müller, H.-H. und Petzel, T., Galvanotechnik, 77, 561-567, 1986.

[KREY95] Kreye, H., Müller, F., Lang, K., Isheim, D. und Hentschel, T., Z. Metallkd., 86, 184-189, 1995.

[KRIL98] Krill, C. E. und Birringer, R., Phil. Mag. A, 77 (3), 621-640, 1998.

[LARS 99] Larson, D. J., Foord, D. T., Petford-Long, A. K., Cerezo, A. und Smith, G. D. W., Nanotechnology, http://www.iop.org/Journals/na, 10, 45-50, 1999.

[LEE 91] Lee, K. K. und Nash, P., in Phase Diagrams of Binary Nickel Alloys, P. Nash (Ed.), ASM International, Materials Park, Ohio, 1991.

[MAED70] Maeda, H., Trans. Nat. Res. Inst. Met., 12 (6), 211-221, 1970.

[MASS90] Massalsky, T. B. (Hrsg.), Binary Alloys Phase Diagrams, ASM International, Metals Park, Ohio, 1990.

[MEHT95] Mehta, S. C., Smith, D. A. und Erb, U., Mater. Sci. Engin. A, 204, 227232, 1995.

[MICH99] Michels, A., Krill, C. E., Ehrhardt, H., Birringer, R. und Wu, D. T., Acta mater., 47 (7), 2143-2152, 1999.

[MILL89] Miller, M. K. und Smith, G. D. W., Atomprobe Microanalysis: Principles and Applications to Materials Problems, Materials Research Society, Pittsburgh, 1989.

[MILL96] Miller, M. K., Cerezo, A., Hetherington, M. G. und Smith, G. D. W., Atom Probe Field Ion Microscopy, Clarendon Press, Oxford, 1996.

[MÖLL88] Möller, W., Eckstein, W. und Biersack, J. P., Computer Physics Communications, 51, 355-368, 1988.

[MORI 78] Morikawa, H., Suzuki, T., Terao, T. und Yashiro, Y., Surf. Sci., 75, 538548, 1978.

[MÜLL69] Müller, E. W. und Tsong, T. T., Field Ion Microscopy, Elsevier Publishing Company, New York, 1969. 
[MÜLL96] Müller, F., in Fortschritt-Berichte VDI, Reihe 5 Nr. 427, VDI, Düsseldorf, 1996.

[NAST96] Nastasi, M., Mayer, J. W. und Hirvonen, J. K., Ion-Solid Interactions: Fundamentals and Applications, Cambridge University Press, Cambridge, 1996.

[OSMO92] Osmola, D., Nolan, P., Erb, U., Palumbo, G. und Aust, K. T., phys. stat. sol. (a), 131, 569-575, 1992.

[PALU90] Palumbo, G., Thorpe, S. J. und Aust, K. T., Scri. Metall. et Mater., 24, 1347-1350, 1990.

[REIM67] Reimer, L., Elektronenmikroskopische Untersuchungs- und Präparationsmethoden, Springer-Verlag, Berlin, 1967.

[RIED89] Riedel, W., Funktionelle Chemische Vernicklung, Eugen Leuze Verlag, Saulgau/Württemberg, 1989.

[RIED91] Riedel, W., Electroless Nickel Plating, ASM International, Metals Park, $\mathrm{OH}, 1991$.

[SHIM99] Shim, J.-H., Chung, H.-J. und Lee, D. N., J. Alloys and Compounds, 282, 175-181, 1999.

[STÖR95] Störmer, M. und Krebs, H.-U., J. Appl. Phys., 78 (12), 7080-7087, 1995.

[STÖR98] Störmer, M., Struktur, Mikrostruktur und innere Spannungen laserdeponierter metallischer Legierungen, Dissertation, Universität Göttingen, 1998.

[STUR99] Sturm, K., Fähler, S. und Krebs, H.-U., Pulsed Laser Deposition of Metals in Low Pressure Inert Gas, eingereicht bei Appl. Surf. Sci., 1999.

[STUR0 0] Sturm, K., wird veröffentlicht, Dissertation, Universität Göttingen, 2000.

[SUTT95] Sutton, A. P. und Balluffi, R. W., Interfaces in Crystalline Materials, Clarendon Press, Oxford, 1995.

[VAFA78] Vafaei-Makhsoos, E., Thomas, E. L. und Toth, L. E., Metall. Trans., 9A, 1449-1460, 1978.

[WARR69] Warren, B. E., X-Ray Diffraction, Addison-Wesley Publishing Company, Reading, 1969.

[WRIE73] Wriedt, H. A., Morrison, W. B. und Cole, W. E., Metall. Trans., 4, 14531456, 1973.

[ZHAN98] Zhang, Y. Z., Wu, Y. Y. und Yao, M., J. Mater. Sci. Lett., 17, 37-40, 1998.

[ZIEG85] Ziegler, J. F., Biersack, J. P. und Littmark, U., The Stopping and Range of Ions in Solids, Pergamon Press, New York, 1985. 


\section{Anhang A}

\section{TRIM-Simulationen zu Fe-Ag-Schichten}

Um die Größen von Sputterausbeuten, die bei der gepulsten Laserdeposition von Fe-AgLegierungen auf W-Spitzen eine Rolle spielten, abzuschätzen, wurden Monte-CarloSimulationen durchgeführt. Der Beschuss von Fe- und Ag-Schichten mit Fe- und AgIonen wurde mit Hilfe des Programms SRIM-2000, Version 10, auf konventionellen PCs simuliert. Eine vollständige Beschreibung des Programms ist in [ZIEG85, IBM96] nachzulesen. Der Kern der Software ist das weithin bekannte Programm TRIM (Transport of Ions in Matter) [BIER80, ZIEG85, NAST96]. Das Programm simuliert die Wechselwirkung von Ionen mit amorphen Festkörpern. Das heißt, es werden einzelne Stöße zwischen Ionen und Atomen im Festkörper bei zufälliger Wahl der Stoßwinkel simuliert. Bei einem Stoß wird ein Teil der kinetische Energie des Ions an das Atom übertragen. Je nach Größe der verbleibenden Energien - einem herausgeschlagenen Atom wird noch eine Bindungsenergie $\mathrm{E}_{1}$ (Lattice Binding Energy) abgezogen - können beide Teilchen dann weitere Stoßprozesse durchlaufen. Dadurch entstehen ganze Stoßkaskaden. Bei Unterschreitung einer zu definierenden Energie $\mathrm{E}_{\mathrm{d}}$ (Displacement Energy) werden Stoßkaskaden beendet. Gelangt ein Teilchen an die Oberfläche des Festkörpers, so wird anhand einer Schwellenergie $E_{\mathrm{s}}$ (Surface Binding Energy) festgelegt, ob ein Teilchen den Festkörper verlässt oder nicht. Als Schwellwerte dienen üblicherweise die Sublimationsenthalpien des Festkörpers. Durch Mittelung über eine Vielzahl von simulierten Einzelbeschüssen werden dann Größen wie Eindringtiefen, der Anteil rückgestreuter Ionen und herausgeschlagener Atome aus dem Festkörper (Sputterausbeute) bestimmt.

Die Autoren des Programms nennen im Handbuch als untere Grenze für Ionenenergien $10 \mathrm{eV} / \mathrm{amu}$. Die hier verwendeten Energien von ca. $140 \mathrm{eV}$ pro Ion liegen damit außerhalb dieses Bereiches. Allerdings sind die angegebenen Grenzen nicht scharf. Andere Autoren, und auch einer der Programmautoren selbst, geben als kritische untere Grenze einen Wert von 1 eV/amu an [MÖLL88]. Einer Anwendung des Programms scheint deshalb prinzipiell nichts im Wege zu stehen. Die Interpretation der Ergebnisse sollte dabei aber vielleicht eher einen qualitativen als quantitativen Charakter haben.

Die wesentlichen Parameter, die für die Simulationen verwendet wurden, sind in Tab. A.1 aufgelistet. Es wurden jeweils 50000 Durchläufe pro Simulation berechnet. Als Schichtdicken wurden $100 \mathrm{~nm}$ angesetzt.

\begin{tabular}{|l|c|c|}
\hline & Fe & Ag \\
\hline Masse [amu] & 55,84 & 107,87 \\
\hline Dichte [g/cm $\left.{ }^{3}\right]$ & 7,866 & 10,473 \\
\hline $\mathbf{E}_{\mathbf{d}}$ (Displacement Energy) [eV] & 25 & 25 \\
\hline $\mathbf{E}_{\mathbf{l}}$ (Lattice Binding Energy) [eV] & 3 & 3 \\
\hline $\mathbf{E}_{\mathbf{s}}$ (Surface Binding Energy) [eV] & 4,34 & 2,97 \\
\hline
\end{tabular}

Tab. A.1 Parameter für TRIM-Simulationen. 


\section{Ergebnisse der TRIM-Simulationen}

Die Ergebnisse der Simulationen sind hier in tabellarischer Form zusammengefasst. Neben dem Depositionswinkel $\varphi$ sind aufgeführt die Sputterausbeute $Y(\varphi)$, der Anteil rückgestreuter Ionen $Y_{b s}(\varphi)$, die effektive Sputteraubeute $Y_{\text {eff }}(\varphi)=Y(\varphi) / Y_{b s}(\varphi)$ und die auf 1 normierte Depositionsrate $\mathrm{R}(\varphi)=\left(1-\mathrm{Y}(\varphi)-\mathrm{Y}_{\mathrm{bs}}(\varphi)\right) /\left(1-\mathrm{Y}(0)-\mathrm{Y}_{\mathrm{bs}}(0)\right)$.

\begin{tabular}{|c|c|c|c|c|}
\hline$\varphi$ & $\mathbf{Y}(\varphi)$ & $\mathbf{Y}_{\mathrm{bs}}(\varphi)$ & $\mathbf{Y}_{\mathrm{eff}}(\varphi)$ & $\mathbf{R}(\varphi)$ \\
\hline 0 & 0,2523 & 0,0001 & 0,2523 & 1 \\
\hline 10 & 0,2339 & 0,0003 & 0,2340 & 1,0243 \\
\hline 20 & 0,2391 & 0,0028 & 0,2398 & 1,0140 \\
\hline 30 & 0,2666 & 0,0137 & 0,2703 & 0,9627 \\
\hline 40 & 0,3097 & 0,0387 & 0,3222 & 0,8715 \\
\hline 50 & 0,3673 & 0,0844 & 0,4012 & 0,7334 \\
\hline 60 & 0,4242 & 0,1713 & 0,5119 & 0,5410 \\
\hline 70 & 0,4470 & 0,2948 & 0,6339 & 0,3453 \\
\hline 80 & 0,3870 & 0,4587 & 0,7150 & 0,2063 \\
\hline
\end{tabular}

Tab. A.2 Ergebnisse für Deposition $136 \mathrm{eV}-\mathrm{Fe}$ auf Fe.

\begin{tabular}{|c|c|c|c|c|}
\hline$\varphi$ & $\mathbf{Y}(\varphi)$ & $\mathbf{Y}_{\mathrm{bs}}(\varphi)$ & $\mathbf{Y}_{\mathrm{eff}}(\varphi)$ & $\mathbf{R}(\varphi)$ \\
\hline 0 & 0,4937 & 0,0002 & 0,4938 & 1 \\
\hline 10 & 0,4670 & 0,0009 & 0,4674 & 1,0513 \\
\hline 20 & 0,4836 & 0,0045 & 0,4858 & 1,0114 \\
\hline 30 & 0,5245 & 0,0168 & 0,5335 & 0,9062 \\
\hline 40 & 0,5865 & 0,0449 & 0,6141 & 0,7282 \\
\hline 50 & 0,6639 & 0,0932 & 0,7321 & 0,4800 \\
\hline 60 & 0,7293 & 0,1807 & 0,8902 & 0,1777 \\
\hline 70 & 0,7441 & 0,3031 & 1,0677 & $-0,0932$ \\
\hline 80 & 0,6677 & 0,4643 & 1,2464 & $-0,2608$ \\
\hline
\end{tabular}

Tab. A.3 Ergebnisse für Deposition $144 \mathrm{eV}-\mathrm{Ag}$ auf Ag. 


\begin{tabular}{|c|c|c|c|c|}
\hline$\varphi$ & $\mathbf{Y}(\varphi)$ & $\mathbf{Y}_{\text {bs }}(\varphi)$ & $\mathbf{Y}_{\text {eff }}(\varphi)$ & \\
\hline 0 & 0,8484 & 0,0748 & 0,9170 & \\
\hline 10 & 0,8267 & 0,0812 & 0,8998 & \\
\hline 20 & 0,8078 & 0,0953 & 0,8929 & \\
\hline 30 & 0,8032 & 0,1226 & 0,9154 & \\
\hline 40 & 0,8029 & 0,1693 & 0,9665 & \\
\hline 50 & 0,8010 & 0,2383 & 1,0517 & \\
\hline 60 & 0,7748 & 0,3177 & 1,1356 & \\
\hline 70 & 0,7097 & 0,4264 & 1,2372 & \\
\hline 80 & 0,5958 & 0,5568 & 1,3442 & \\
\hline
\end{tabular}

Tab. A.4 Ergebnisse für Deposition $136 \mathrm{eV}-\mathrm{Fe}$ auf Ag.

\begin{tabular}{|c|c|c|c|l|}
\hline$\varphi$ & $\mathbf{Y}(\varphi)$ & $\mathbf{Y}_{\text {bs }}(\varphi)$ & $\mathbf{Y}_{\text {eff }}(\varphi)$ & \\
\hline 0 & 0,0661 & 0 & 0,0661 & \\
\hline 10 & 0,0509 & 0 & 0,0509 & \\
\hline 20 & 0,0518 & 0 & 0,0518 & \\
\hline 30 & 0,0768 & 0 & 0,0768 & \\
\hline 40 & 0,1272 & 0,0006 & 0,1273 & \\
\hline 50 & 0,2161 & 0,0089 & 0,2180 & \\
\hline 60 & 0,3409 & 0,0491 & 0,3585 & \\
\hline 70 & 0,4365 & 0,1550 & 0,5166 & \\
\hline 80 & 0,4620 & 0,3602 & 0,7221 & \\
\hline
\end{tabular}

Tab. A.5 Ergebnisse für Deposition $144 \mathrm{eV}-\mathrm{Ag}$ auf Fe. 


\section{Anhang B}

\section{Deposition von Fe-Ag-Schichten auf W-Spitzen unter Ar-Atmosphäre}

Mit Hilfe von Ar-Gas wurde versucht, insbesondere den hochenergetischen Anteil der Ionen bei der PLD zu verringern. PLD-Experimente unter Ar-Gas-Atmosphäre [STUR99] zeigten, dass bei Ar-Partialdrücken von $4 \times 10^{-2} \mathrm{hPa}$ die Energien der deponierten Ionen reduziert werden konnten und auch die Sputtereffekte deutlich verringert waren.

Es wurden Fe-Ag-Schichten mit Hilfe der PLD unter Ar-Atmosphäre auf WolframSpitzen aufgebracht. Die Präparation der W-Spitzen, die Versuchsanordnung und die Versuchsbedingungen - mit Ausnahme des Ar-Partialdruckes - waren mit denen bei der Deposition unter UHV-Bedingungen, wie sie in Kap. 2.1 beschrieben sind, identisch. Die Versuche ergaben ein überaschendes Bild: Es wurden Schichtdicken auf den Spitzen gefunden, die erheblich größer waren als die der planaren Referenzschichten. Auffällig war ferner, dass die geometrische Form der Schichten sehr symmetrisch und die Kappen teilweise ideal halbsphärenförmig waren. In Abb. B.1 sind TEM-Aufnahmen von zwei Spitzen vor und nach der Deposition dargestellt. Die Schichtdicken der planaren Referenzschichten betrugen in diesen Fällen $47 \mathrm{~nm}$ und $1 \mathrm{~nm}$. Auf den Spitzen wurden die Schichtdicken aus den TEM-Aufnahmen auf ca. $120 \mathrm{~nm}$, und $25 \mathrm{~nm}$ abgeschätzt.

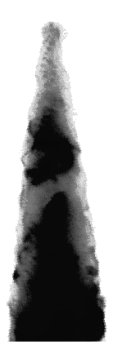

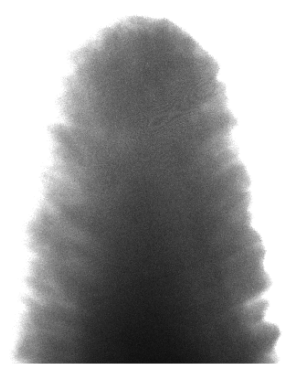

(A)

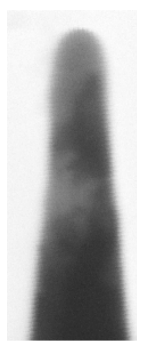

(B)

Abb. B.1 Fe-Ag-Schichten deponiert unter Ar-Atmosphäre $\left(4 \times 10^{-2} \mathrm{hPa}\right)$ auf TAP-Spitzen. Es sind jeweils die Spitzen vor und nach der Beschichtung dargestellt. Die Schichtdicken der planaren Referenzschichten betragen (A) $47 \mathrm{~nm}$; (B) $1 \mathrm{~nm}$. Die Schichten auf den Spitzen sind erheblich dicker als die planaren Referenzschichten.

Die FIM-Abbildung einer Spitze zeigte keine besonderen Kontraste oder Strukturen. Es konnte aber ein stark gestörter Pol beobachtet werden. Feldverdampfende Ebenen waren sichtbar. Ein FIM-Bild dieser Spitze ist in Abb. B.2 zu sehen. Der genannte Pol ist durch Pfeile im Bild markiert. Der Ag-Gehalt der planaren Referenzschicht betrug in diesem Fall ca. 10 At.\%.

Der Versuch einer TAP-Analyse lieferte lediglich wenige Ionen bevor die Spitze abriss. Es konnten $2600 \mathrm{Fe}$-Ionen detektiert werden. Hinweise auf Ag-Ionen gab es keine. Das Erbebnis dokumentiert jedoch, dass Eisen auf den Spitzen deponiert wurde. 


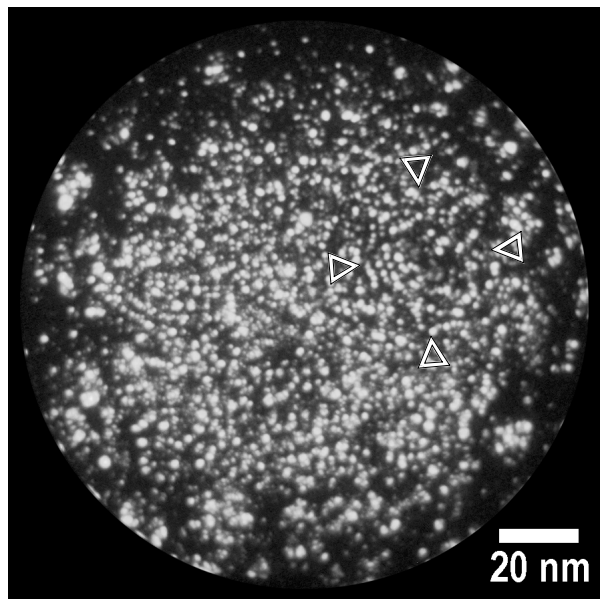

Abb. B.2 FIM-Aufnahme einer Fe-Ag-Schicht, die unter Ar-Atmosphäre auf einer W-Spitze deponiert wurde. Nur schemenhaft zu erkennen ist ein Pol, der durch Pfeile markiert ist.

Eine Ursache für das abnormale Schichtwachtum kann nur vermutet werden. Es könnte beispielsweise sein, dass durch die Gas-Atmosphäre die Zahl der ionisierten Teilchen so groß ist, dass durch die Ladungstrennung im Plasma-Strahl [KREB97] hohe elektrische Felder entstehen, so dass Ionen auf die geerdete Spitze hin beschleunigt werden. 


\section{Anhang C}

\section{Bestimmung von Phosphor-Konzentrationen in Korngrenzen}

Bei der Ermittlung der P-Konzentrationen in den Korngrenzen der Ni-P-Legierungen gilt es folgendes zu berücksichtigen: Die zu untersuchenden Strukturen sind so klein, dass eine gewisse Unschärfe zwischen Konzentration und räumlicher Auflösung ins Spiel kommt. Die hier gezeigten Konzentrationsprofile wurden i. d. R. mit zylinderförmigen Testvolumina erstellt, die sehr flach $(0,4 \mathrm{~nm})$ ausfielen, um die Konzentrationsverläufe durch die Korngrenzen möglichst gut wiederzugeben. Dennoch sind die Korngrenzen der kleinen Körner auch auf der Breite der Testvolumina nicht planar. Relativ breite Zylinder mit Durchmessern von $4 \mathrm{~nm}$ liefern daher zwar Profile mit geringem Rauschen, dafür aber auch scheinbar geringere Konzentrationen und breitere Segregationszonen. Um die Breite der Segregationszonen zu bestimmen wurden daher möglichst kleine Volumina (mit 2-3 nm Durchmesser) gewählt. In Abb. C.1 sind zwei Konzentrationsprofile mit unterschiedlichen Analyszylindern durch dieselbe Korngrenzen gezeigt. Aus dem oberen Profil resultiert bei einem Analysezylinder mit 4 $\mathrm{nm}$ Durchmesser eine $2 \mathrm{~nm}$ breite Korngrenze mit einer Konzentration von ca. 9 At.\% P. Mit Hilfe des kleineren Analysezylinders ergeben sich eine (realistischere) Breite von ca. 0,5 nm und ein P-Gehalt von 15 At.\%.
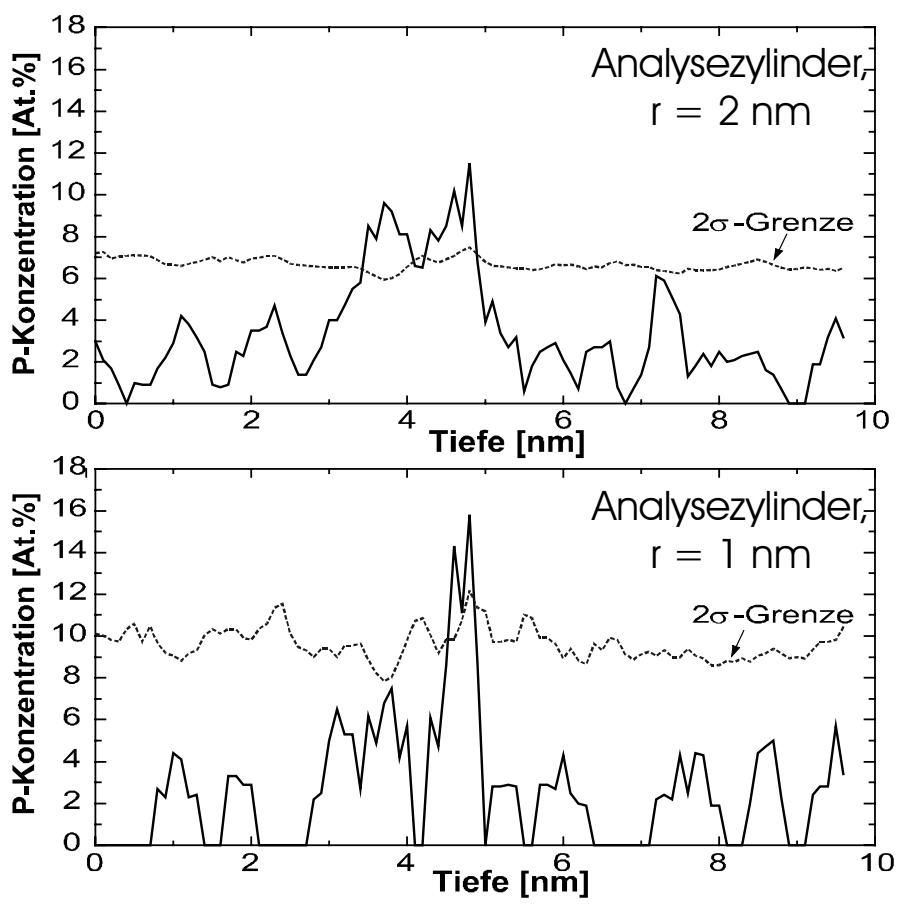

Abb. C.1 Konzentrationsprofile durch die Korngrenze einer Ni3,6 At.\% P-Legierung (getempert bei $\left.320^{\circ} \mathrm{C} \backslash 1 \mathrm{~h}\right)$. Beide Profile wurden an derselben Position ermittelt, aber mit unterschiedlich großen Test-Analysezylindern.

Außerdem muss berücksichtigt werden, dass die räumliche Auflösung der TAP am besten in Richtung der Analyse ist. Bei der Auswahl von Korngrenzen für präzise Konzentrationsanalysen wurden daher solche ausgewählt, die möglichst senkrecht zur Analyserichtung der TAP lagen. Eine Abweichung von dieser Orientierung führt ansonsten auch zu scheinbar breiteren Peaks aufgrund von lokalen Vergrößerungseffekten bei der TAP-Analyse [BLAV96]. 
Für die Bestimmung des Korngrenzen-Exzesses hingegen spielen die genannten Einschränkungen nur eine untergeordnete Rolle, da er von der Breite der Korngrenzen unabhängig ist. 


\section{Worte des Dankes}

Die vorliegende Arbeit entstand während meiner Tätigkeit als wissenschaftlicher Mitarbeiter am Institut für Materialphysik an der Universität Göttingen. Viele nette Menschen, Kollegen und Freunde haben mit zu ihrem Gelingen und dazu beigetragen, dass ich diese Zeit in guter Erinnerung behalten werde.

Mein erster Dank gilt Herrn Prof. Dr. Reiner Kirchheim für sein in mich gesetztes Vertrauen, für die große Freiheit, die er mir bei meiner Forschungstätigkeit gelassen hat, für seine Ratschläge und für die Unterstützung in vielen Dingen. So war etwa die Möglichkeit für mich im Rahmen meiner Arbeit einen Frankreich-Aufenthalt realisieren zu können sehr motivierend.

Gedankt sei auch allen Mitgliedern der Groupe de Métallurgie Physique von Prof. Didier Blavette an der Université de Rouen in Frankreich für die fruchtbare Zusammenarbeit und die Einweisung in die Geheimnisse der Tomographischen Atomsonde.

$\mathrm{Zu}$ besonderem Dank verpflichtet bin ich Dr. Guido Schmitz für seine offene und motivierende Art, für zahlreiche kritische und fruchtbare Diskussionen und eine sehr produktive Zusammenarbeit, die stets viel Freude gemacht hat.

Dank sage ich auch Dr. Tala'at Al-Kassab für interessante Diskussionen seine stete Hilfsbereitschaft und eine schöne Zeit im gemeinsamen Büro.

Den weiteren Mitgliedern der FIM-Gruppe, Tobias Jeske, Christian Lang, Jörg Schleiwies, Philipp Kesten, Christian Kluthe und Pyuck-Pa Choi danke ich für die gute Zusammenarbeit und die gute Arbeitsatmosphäre. Die „Teerunde“, die wohl immer mehr zur Kaffeerunde wird, lieferte auch immer wieder anregende Diskussionen.

Allen Mitarbeitern, die am Teilprojekt A20 im Sonderforschungsbereich 345 mitgewirkt haben, insbesondere Dr. Michael Störmer, danke ich für die Zusammenarbeit.

Danken möchte ich auch allen Angehörigen des Instituts für Materialphysik, die dafür gesorgt haben, dass das produktive Arbeiten und Experimentieren möglich war und die durch ihren Einsatz, ihre Hilfsbereitschaft und den unkomplizierten Umgang das Arbeiten angenehm gemacht haben.

Besonders danken möchte ich auch meinen Freunden und Freundinnen, die mich insbesondere in schwierigen Phasen unterstützt und mir geholfen haben.

Meiner Familie und besonders meiner Lebensgefährtin Kristina Köhler danke ich von ganzem Herzen für ihre Geduld und ihr großes Verständnis, dass sie mir entgegengebracht haben, und für die Liebe, das Vertrauen und die Geborgenheit, die eine gute Basis bilden nicht nur für eine erfolgreiche Arbeit :-)) 



\section{Lebenslauf}

Ich wurde am 09.02.1968 in Heide in Holstein als Sohn meiner Eltern Sigrid Färber, geborene Tegge, und Klaus Färber geboren.

Ich wuchs auf in der Gemeinde Lohe-Rickelshof, wo ich auch von 1972 bis 1976 die Grundschule besuchte. Danach wechselte ich auf das Werner-Heisenberg-Gymnasium in Heide. Im Juni 1987 erlangte ich die Allgemeine Hochschulreife.

Von 1987 bis 1989 war ich Soldat bei der Bundeswehr in der Topographie Batterie 600 in Rendsburg. Im Rahmen einer Reserveoffizierslaufbahn wurde ich zum Vermessungsoffizier ausgebildet. Zeitgleich studierte ich im Teilzeitstudium Betriebsinformatik an der Fern-Universität in Hagen.

Zum Wintersemester 1989 nahm ich das Studium der Physik an der Universität Göttingen auf. Dort legte ich 1991 die Diplomvorprüfung ab. Am Institut für Metallphysik der Universität Göttingen fertigte ich unter der Anleitung von Herrn Prof. Dr. Reiner Kirchheim meine Diplomarbeit zum Thema „Mikrocharakterisierung stark übersättigter und rasch abgeschreckter CuCo-Legierungen“ an. Im Februar 1996 legte ich dann die Diplomprüfung ab.

Seit April 1996 arbeite ich als wissenschaftlicher Mitarbeiter an der Universität Göttingen im Sonderforschungsbereich 345 bei Herrn Prof. Dr. Reiner Kirchheim am (heutigen) Institut für Materialphysik.

Boye Färber

E-Mail: boye@dr-faerber.de 